\title{
Impacts of climate change on interacting plant and bird species - A trait-based perspective
}

\author{
Dissertation \\ for attaining the $\mathrm{PhD}$ degree \\ submitted to the Faculty of Biological Sciences \\ of the Johann Wolfgang Goethe University \\ in Frankfurt am Main \\ by \\ Larissa Nowak \\ from Aalen, Germany
}

Frankfurt 2021

(D 30) 
Accepted by the faculty of Biological Sciences of the Johann Wolfgang Goethe University as a dissertation.

Dean: Prof. Dr Sven Klimpel

First reviewer: PD Dr Matthias Schleuning

Second reviewer: Prof. Dr Claus Bässler

Date of disputation: 


\section{Table of contents}

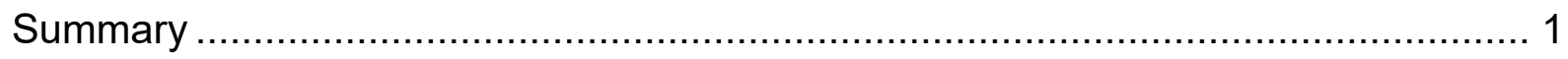

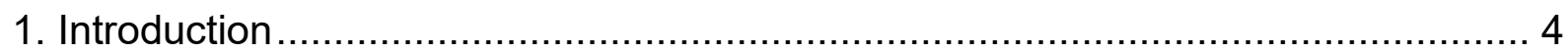

1.1. Biodiversity under contemporary climate change ....................................... 4

1.2. The relevance of biotic interactions ........................................................ 5

1.3. Estimating climate-change impacts on biodiversity ..................................... 6

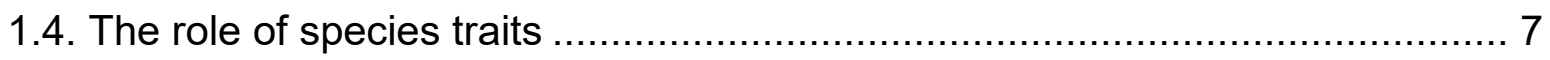

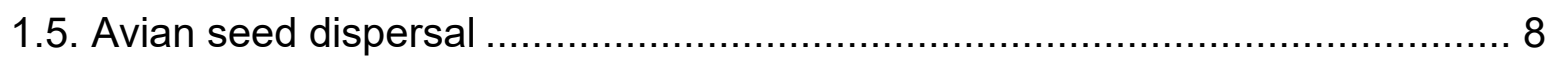

1.6. Tropical elevational gradients................................................................ 9

2. Dissertation structure and research questions............................................ 10

2.1 How do species' sensitivity to climate change and their adaptive capacity covary? (Q1)

2.2 How will future climate change affect the functional correspondence of interacting species? (Q2)

2.3 Is avian seed dispersal sufficient for plants to track future temperature change

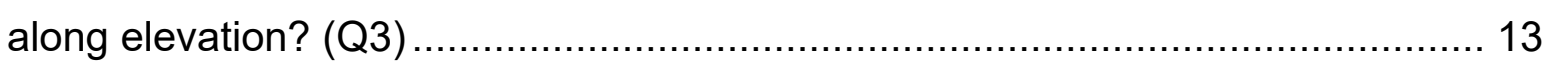

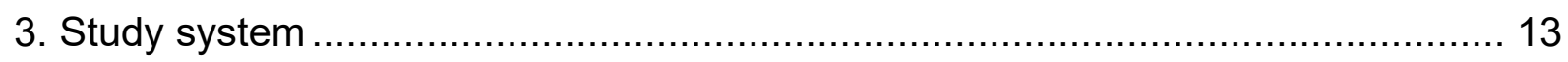

4. Data and methodological approaches ………............................................... 15

4.1. Elevational ranges of plants and birds..................................................... 15

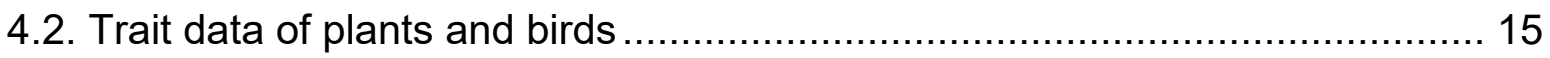

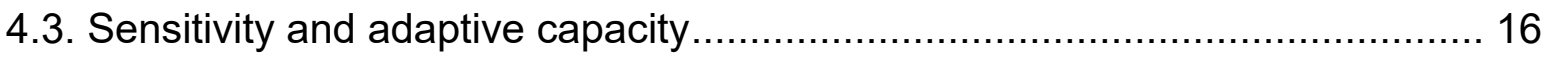

4.4. Projecting the functional diversity of interacting species ............................. 17

4.5. Trait-based simulations of plants' climate-tracking ability ........................... 18

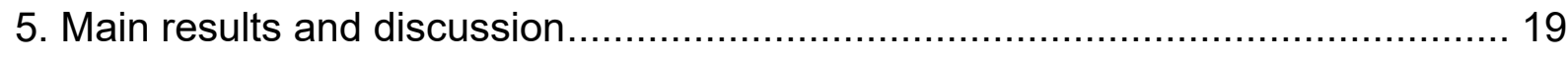

5.1. Independent variation of avian sensitivity to climate change and adaptive

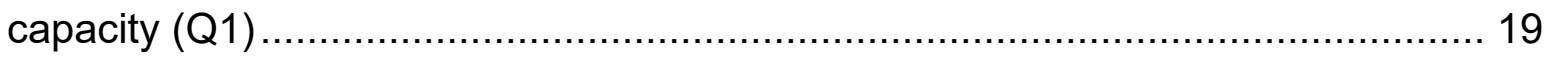

5.2. Low functional correspondence of interacting species under a scenario of range

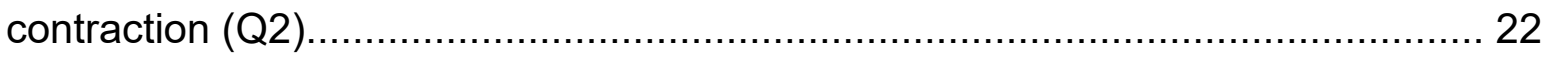


5.3. Avian seed dispersal might be insufficient for plants to track future temperature

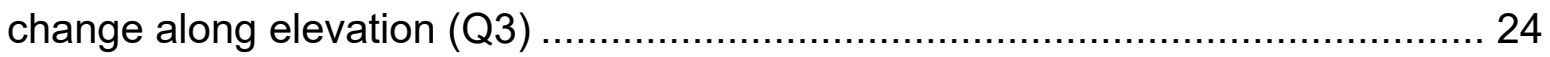

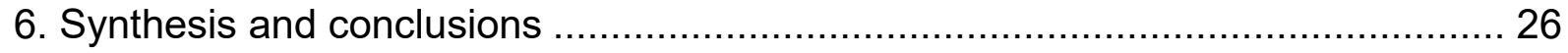

6.1. Trait-based approaches to study the impacts of climate change on interacting species

6.2. Climate change and interacting species along tropical elevational gradients

Zusammenfassung

References .

Acknowledgements 54

Appendices. 56

A1: Independent variation of avian sensitivity to climate change and adaptive capacity along a tropical elevational gradient. 56 A2: Projecting consequences of global warming for the functional diversity of fleshyfruited plants and frugivorous birds along a tropical elevational gradient 83 A3: Avian seed dispersal may be insufficient for plants to track future temperature change on tropical mountains. 106

A4: Curriculum Vitae 133

A5: Publications and scientific presentations 137 


\section{Summary}

Global biodiversity is changing rapidly and contemporary climate change is an important driver of this change. As climate change continues, the challenge is to understand how it may affect the future of biodiversity. This is relevant to informing policy and conservation, but it requires reliable future projections of biodiversity. Biodiversity is the variety of life on Earth which includes the diversity of species. The species on Earth are linked in diverse networks of biotic interactions. Interacting species can respond differently to climate change. This can cause spatial or temporal mismatches between interacting species and result in secondary extinctions of species that lose obligate interaction partners. Yet, accounting for biotic interactions in biodiversity projections remains challenging. One way to address this challenge is the use of trait-based approaches because the impact of climate change on interacting species is influenced by species' functional traits, i.e., measurable characteristics of the species that influence their abiotic and biotic interactions. First, species' functional traits influence how species respond to climate change. Second, they influence whether the species find compatible interaction partners in reshuffled species assemblages under climate change. Thus, the overarching aim of this dissertation was to explore how trait-based approaches can increase our understanding of how climate change might affect interacting species. For this, I focussed on interactions between fleshy-fruited plants and avian frugivores along a tropical elevational gradient.

I investigated three principal research questions. First, I investigated how traits related to the sensitivity of avian frugivores to climate change and their adaptive capacity vary along elevation and covary across species. I combined estimates of species' climatic niche breadth (approximating species' sensitivity) with traits influencing species' dispersal ability, dietary niche breadth and habitat niche breadth (aspects of species' adaptive capacity). Species' climatic niche breadth increased with increasing elevation, while their dispersal ability and dietary niche breadth decreased with increasing elevation. Across species, there was no significant relationship of the sensitivity of the avian frugivores to climate change and their adaptive capacity. The opposing patterns of species' sensitivity to climate change and their adaptive capacity along elevation imply that species from assemblages at different elevations may respond differently to climate change. The independence between species' sensitivity and adaptive capacity suggests that it is important to account for both sensitivity and adaptive capacity to fully understand how climate change might affect biodiversity. 
Second, I assessed how climate change might influence the co-occurrence of interaction partners with compatible traits, i.e., the functional correspondence of interacting species. I integrated future projections of species' elevational ranges considering different vertical dispersal scenarios with analyses of the functional diversity of interacting species assemblages. The functional correspondence of fleshyfruited plants and avian frugivores was lowest if plant and bird species were projected to contract their ranges towards higher elevations in response to increasing temperatures. Contrastingly, if species were projected to expand their ranges upslope, the functional correspondence remained close. The low functional correspondence under a scenario of range contraction indicates that plant species with specific traits might miss compatible interaction partners in future assemblages. This could negatively affect their seed dispersal ability. These results suggest that ensuring the integrity of biotic interactions under climate change requires that species can shift their ranges upslope unlimitedly.

Third, I examined whether avian seed dispersal is sufficient for plants to track future temperature change along the elevational gradient. With a trait-based modelling approach, I simulated seed-dispersal distances avian frugivores can provide to fleshyfruited woody plant species and quantified the number of long-distance dispersal events the plant species would require to fully track projected temperature shifts along elevation. Most plant species were projected to require several long-distance dispersal events to fully track the projected temperature shifts in time. However, the number of required long-distance dispersal events varied with the degree of trait matching and plant species' traits. These findings suggest that avian seed dispersal is insufficient for plants to track future temperature change along the elevational gradient as woody plant species might not be able to undergo several consecutive long-distance dispersal events within a short time window, due to their long maturation times. These results also imply that the ability of bird-dispersed plant species to track climate change is associated with the specialization of the seed dispersal system and with plant species' traits.

Trait-based approaches are promising tools to study impacts of climate change on interacting species. The trait-based approaches that I have developed in this thesis are applicable more widely, e.g., to other types of biotic interactions, or to assess the effects of other drivers of global change. Moreover, these approaches may be further developed to model changes in biotic interactions under global change more 
dynamically. Taken together, I have shown how a trait-based perspective could help to account for biotic interactions in biodiversity projections. The development of such approaches and the gained knowledge are urgently needed to facilitate the conservation of biodiversity in a rapidly changing world. 


\section{Introduction}

\subsection{Biodiversity under contemporary climate change}

Biodiversity, i.e., the diversity of life on Earth, is crucial for the functioning of ecosystems and the potential of nature to contributing to people (Díaz et al. 2019). Nevertheless, global biodiversity is changing rapidly. Among the main drivers of this change are land-use and sea-use change, pollution, overexploitation, invasive species and contemporary climate change (Díaz et al. 2019). The global climate is changing fast. Since the preindustrial time (1850-1900), the average global surface temperature has increased by approximately one degree Celsius (IPCC 2014, 2018). This is linked to anthropogenic greenhouse gas emissions and is projected to continue if greenhouse gas concentrations in the atmosphere rise further (IPCC 2014, 2018).

Among the prevalent biotic responses to climate change is spatial redistribution of species (Pecl et al. 2017). Globally, species are shifting their geographic ranges to higher latitudes and higher elevations to track shifts in their suitable climate (Chen et al. 2011; Lenoir et al. 2020). Individual species can thereby differ greatly in the magnitude and pace of these range shifts (Moritz et al. 2008; Chen et al. 2011). This spatial redistribution of life on Earth can reshuffle the composition of species assemblages and alter local to global-scale biodiversity patterns ( $\mathrm{Pecl}$ et al. 2017). Another well-documented biotic response to climate change is phenological change, i.e., shifts in the timing of recurrent events in a species' life cycle (Parmesan \& Yohe 2003; Kharouba et al. 2018). The impacts of climate change on biodiversity are projected to continue (Urban 2015; Trisos et al. 2020). If climate change advances unmitigated, many species globally will experience climate conditions beyond those they have previously encountered (Mahony et al. 2017; Trisos et al. 2020). The challenge is to understand how that might affect the future of biodiversity.

Future projections of global biodiversity can provide valuable information in this respect. For example, future projections of biodiversity enable assessing the potential consequences of different scenarios of future climate change, such as limiting global warming to $1.5^{\circ} \mathrm{C}$ or failing to do so (IPCC 2018; Warren et al. 2018). Such knowledge may inform policy decisions and help to underpin the urgency of climate-change mitigation (IPCC 2018). Furthermore, knowledge of possible future trajectories of biodiversity can inform biodiversity conservation. For example, based on future projections of biodiversity, it is possible to determine the extent of protected areas required for preventing biodiversity loss (Hannah et al. 2020) or to evaluate the 
efficiency of networks of protected areas in the face of global change (Bagchi et al. 2013; Gillingham et al. 2015). However, informing policy and conserving biodiversity requires reliable future projections of biodiversity (Urban et al. 2016).

\subsection{The relevance of biotic interactions}

We can only fully understand how climate change may affect biodiversity if we consider biotic interactions (Zarnetske et al. 2012). Species are entangled in diverse networks of biotic interactions. In antagonistic interactions, one partner benefits while the other is negatively affected, such as in plant-herbivore or predator-prey interactions (Abrams 2000; Stam et al. 2014). On the other hand, in mutualistic interactions, all involved interaction partners benefit, such as in plant-pollinator or plant-frugivore interactions (Bawa 1990; Jordano et al. 2011). Importantly, species that are linked to each other by biotic interactions might respond differently to climate change. Species can differ in their climatic niches, with relative increases of warm-adapted compared to coldadapted species under rising temperatures (Bowler et al. 2017). Furthermore, species can differ in their ability to track shifts in their suitable climate, due to differences in their dispersal ability or life history (Chen et al. 2011; Lenoir \& Svenning 2015; Sunday et al. 2015; Lenoir et al. 2020). Short-lived species, for instance, seem to better track contemporary climate change than long-lived species (Lenoir et al. 2020). Species might as well differ in their ability to evolve wider tolerances or respond to climate change through phenotypic plasticity or behavioural changes (Dawson et al. 2011). Such differences can cause temporal or spatial mismatches in the abundance or the occurrence of interacting species (Schweiger et al. 2008; Kharouba et al. 2018; Schleuning et al. 2020). Well-known examples are changes in the phenological synchrony of interacting species, such as in the timing of the growing season of plants and the migration of herbivorous animals (Post \& Forchhammer 2008), or, between peak insect abundance and avian egg-laying time (Renner \& Zohner 2018). Similarly, spatial mismatches have been predicted for plant-herbivore and plant-pollinator interactions under future climate change (Schweiger et al. 2008; Sales et al. 2021b). Temporal or spatial mismatches can have negative consequences for interacting species. Mismatches between interacting plant and herbivore species, for example, can cause a reduction in the reproductive success of the herbivore (Post \& Forchhammer 2008). The loss of an obligate interaction partner can cause secondary extinction of a species, unless the species can establish novel interactions (Colwell et 
al. 2012; Brodie et al. 2014). This might even trigger climate-induced extinction cascades across several trophic levels (Brodie et al. 2014; Schleuning et al. 2016). Therefore, considering biotic interactions is crucial to project the future of biodiversity.

\subsection{Estimating climate-change impacts on biodiversity}

Impacts of future climate change on biodiversity are often estimated with a focus on species' sensitivity to climate change (e.g., Thuiller et al. 2005, 2011; Bender et al. 2019). A species' sensitivity to climate change is the degree to which a species is affected by climate change (IPCC 2007; Foden et al. 2018), which is related to the species' climatic niche, i.e., climate conditions in which the species can maintain stable populations and evolutionary fitness (IPCC 2007; Algar \& Tarr 2018; Foden et al. 2018). A common approach is to model species' climatic niches from their current occurrences and associated climate variables (Guisan \& Thuiller 2005). Species' potential future distributions can then be predicted from their modelled climatic niche and their projected exposure to future climate change (Thuiller et al. 2005; Elith \& Leathwick 2009).

Another way of estimating potential impacts of future climate change on species are trait-based assessments of species' vulnerability to climate change (Foden et al. $2013,2018)$. These assessments evaluate both species' sensitivity to climate change and different aspects of the species' adaptive capacity to identify species that are most vulnerable to future climate change and areas where many such species occur (Foden et al. 2013; Pacifici et al. 2015). A species' adaptive capacity is defined as the species' ability to adjust to climate change, respond to its consequences and moderate potential damage (IPCC 2007, 2014; Thurman et al. 2020). It is determined by several ecological and evolutionary attributes including the species' ability to shift its range and utilize a wide range of resources (Dawson et al. 2011; Foden et al. 2018).

These approaches to quantify climate-change impacts on biodiversity provide valuable information, but they also have shortcomings. First, studies that focus on species' climatic niches often do not consider aspects of species' adaptive capacity (Araújo \& Guisan 2006; Khaliq et al. 2014; Nunez et al. 2019). However, since it remains untested whether species' sensitivity and adaptive capacity covary, solely focussing on species' sensitivity might overestimate or underestimate the impact of future climate change on biodiversity. Second, while trait-based vulnerability assessments account for aspects of species' adaptive capacity, they do not integrate 
this information into biodiversity projections (Foden et al. 2013; Pacifici et al. 2015). Thus, to improve our understanding of the potential impacts of future climate change on biodiversity, we need to find new ways to account for aspects of species' adaptive capacity as well as biotic interactions in biodiversity projections.

\subsection{The role of species traits}

The impact of climate change on interacting species is influenced by species' functional traits. Functional traits are measurable characteristics of a species that influence the species' abiotic and biotic interactions (Violle et al. 2007; Schleuning et al. 2020). First, species' sensitivity to climate change and their adaptive capacity are related to functional response traits that influence how species respond to environmental change (Hooper et al. 2005; Luck et al. 2012). Functional response traits that influence species' sensitivity to climate change include traits that define species' climatic niche, such as physiological traits related to species' thermal tolerance (Khaliq et al. 2014; Foden et al. 2018). Essential to species' adaptive capacity are functional response traits related to species' dispersal ability as they determine the species' capacity for geographic range shifts under climate change (Foden et al. 2018). For example, the dispersal ability of bird species is related to their wing shape (Dawideit et al. 2009; Santini et al. 2019; Sheard et al. 2020), while the dispersal ability of plant species varies with plant height and seed size (Chen et al. 2019). Equally important for species' adaptive capacity are functional response traits that relate to species' ecological niche breadth because they influence the species' ability to adjust to changes in resource availability (Slatyer et al. 2013). Assessing functional response traits and related species' attributes has the potential to increase our understanding of how species might respond to future climate change.

Second, functional matching traits influence whether species find matching interaction partners in reshuffled species assemblages under climate change. Trait matching between species is an important mechanism influencing biotic interactions (Garibaldi et al. 2015; Bender et al. 2018). Trait matching describes the compatibility of the traits of two species and influences the likelihood of their interaction (Schleuning et al. 2020). A prominent example is the size matching between the flower and the proboscis or the bill in plant-pollinator interactions (Stang et al. 2009; Maglianesi et al. 2014; Weinstein \& Graham 2017). Similarly, the fruit size and the bill size in plantfrugivore interactions are generally closely related (Burns 2013; Dehling et al. 2014b; 
Bender et al. 2018). Functional diversity allows to scale the functional-trait approach to the assemblage level and captures the value, range and relative abundance of such functional matching traits in a species assemblage (Tilman 2001; Díaz et al. 2007). The functional diversity often corresponds between assemblages of interacting species indicating that species with matching traits co-occur (Dehling et al. 2014b; Vollstädt et al. 2017). For example, the functional diversity of fleshy-fruited plant and avian frugivore assemblages are closely associated along elevational gradients in the Neotropics as well as in the Afrotropics (Dehling et al. 2014b; Vollstädt et al. 2017). Future climate change might reshuffle assemblages of interacting species, which could disrupt their functional correspondence (Lindström et al. 2013; Mokany et al. 2015; Pecl et al. 2017). Based on species' matching traits it might be possible to identify ecological systems prone to such functional disruption between interacting species under climate change. Given the promising opportunities offered by a trait-based perspective, the overarching aim of this dissertation is to explore how trait-based approaches can improve understanding of the effects of climate change on interacting species.

\subsection{Avian seed dispersal}

In this dissertation, I focus on the impact of climate change on interacting fleshy-fruited plant and avian frugivore species. Plant-frugivore interactions are globally wide-spread (Kissling et al. 2009; Jordano 2014). Especially, in the tropics, about $90 \%$ of the woody plant species produce fleshy-fruits for animal seed dispersal, and many avian frugivores highly depend on fruits as part of their diet (Kissling et al. 2009; Jordano 2014). The interactions between fleshy-fruited plants and avian frugivores are mutually beneficial (Herrera 2002). On the one hand, the frugivores receive valuable nutrients from the fruits (Moermond \& Denslow 1985; Quintero et al. 2020). On the other hand, the frugivores ingest the seeds and transport them away from the mother plant, thus contributing to the important ecosystem function of seed dispersal (Wang \& Smith 2002). Seed dispersal influences crucial plant processes, such as prevention from sibling competition, avoidance of natural enemies and gene flow between populations (Howe \& Smallwood 1982). Importantly, seed dispersal enables plants to colonize new habitats and to track shifts in their suitable climate during climate change (Howe \& Smallwood 1982). For plant species under climate change, long-distance dispersal is particularly relevant as it determines the farthest distance a seed can be dispersed in 
a seed-dispersal event (Cain et al. 2000; Nathan et al. 2008). However, the plant species' longevity can slow down their dispersal ability (Corlett \& Westcott 2013), as there is a time lag between two consecutive dispersal events in which the plant needs to establish at the new location, mature and produce fruits before it can be dispersed a step further (Wang \& Smith 2002). Long-lived woody plant species, for example, usually take several decades to mature (Salguero-Gómez et al. 2015).

Both plant-frugivore interactions and seed-dispersal distances are influenced by species' morphological traits. First, trait matching, such as between the fruit width and the bill width, influences which bird species feed on which plant species (Dehling et al. 2014b; Bender et al. 2018). Interestingly, the degree of trait matching, i.e., how close the plant and the frugivore traits need to match to make an interaction likely, varies between seed dispersal systems (Bender et al. 2018). This may be due to a certain flexibility of the frugivores to switch between fruit resources (Bender et al. 2017). Second, seed-dispersal dispersal distances are influenced by the body mass of the avian disperser, because large-bodied frugivores tend to transport seeds over greater distances than small-bodied frugivores (Jordano et al. 2007; Wotton \& Kelly 2012). Since avian seed dispersal systems are ecologically highly relevant and influenced by species' traits, it is an excellent study system to assess potential impacts of future climate change on interacting plant and bird species with trait-based approaches.

\subsection{Tropical elevational gradients}

I study the impacts of climate change on interacting fleshy-fruited plants and avian frugivores along an elevational gradient in the Neotropics. Tropical elevational gradients, especially in the Neotropics, are among the most biodiverse regions in the world and host a high diversity of avian frugivores and fleshy-fruited plants (Myers et al. 2000; Jansson \& Davies 2008; Kissling et al. 2009). Elevational gradients are valuable study systems because they display high climate and habitat turnover over relatively small spatial extents (Körner 2000; Sanders \& Rahbek 2012). Specifically, due to their steep decrease in the average temperature with increasing elevation (Mokhov \& Akperov 2006), elevational gradients enable plants to track vertical temperature shifts under climate change (Colwell et al. 2008).

Elevational range shifts of species under climate change might alter elevational biodiversity patterns. At low elevations, biodiversity might decline if the number of species emigrating or going extinct from low elevations exceeds the number of species 
that are warm-adapted and persist (i.e., lowland biotic attrition Colwell et al. 2008; Bender et al. 2019). At mid-elevations, there might be an elevated species turnover as lowland species immigrate and mid-elevation species shift their ranges upslope (Corlett 2011). At high elevations, biodiversity might accumulate due to immigrating species from lower elevations (Steinbauer et al. 2018). However, the extinction risk of high-elevation species might increase, if their suitable climate contracts towards the mountain top (Freeman et al. 2018b). In the tropics, the consequences of elevational range shifts might be particularly pronounced, as latitudinal temperature gradients are shallow and elevational range shifts are therefore more likely (Colwell et al. 2008). Tropical elevational gradients are thus an ideal system for studying impacts of climate change on interacting species.

\section{Dissertation structure and research questions}

In my dissertation, I investigated how future climate change might affect interacting species. I was particularly interested in exploring how trait-based approaches can increase understanding of the potential impacts of future climate change on interacting fleshy-fruited plant and avian frugivore species. I developed three main research questions (Figure 1). The first question (Q1) addresses the ability of avian frugivores to respond to climate change. The second question (Q2) explores potential disruptions of plant-frugivore interactions under climate change. Finally, the third question (Q3) examines the ability of fleshy-fruited plants to respond to climate change through longdistance dispersal mediated by plant-frugivore interactions. To address these questions, I developed new concepts and combined trait-based approaches with estimates of species' climatic niches and future projections of species' elevational ranges. I structured my dissertation into three research articles, of which one is published (Nowak et al. 2019; appendix A2) and two are submitted (appendices A1, A3). 

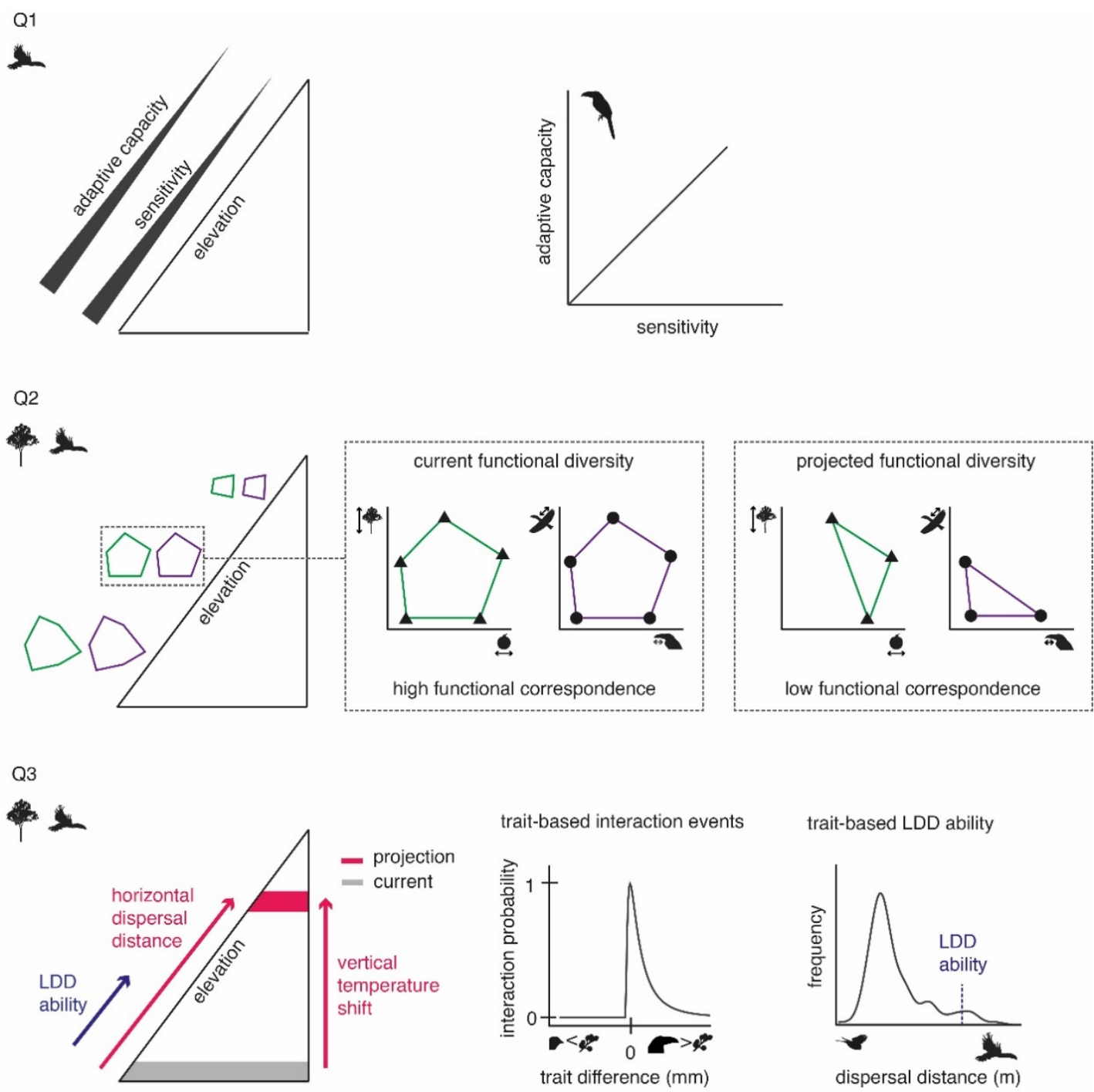

Figure 1: Schematic representation of the dissertation structure. I developed three main research questions to study potential impacts of climate change on interacting plant and bird species with trait-based approaches. Q1: How do species' sensitivity to climate change and their adaptive capacity vary along elevation and covary across avian frugivore species? Q2: How will future climate change affect the functional correspondence of interacting plants and birds? Q3: Is avian seed dispersal sufficient for plants to track future temperature change along elevation?

Explanations: Narrowing bars along elevation in Q1 indicate expected variation of species' sensitivity to climate change and their adaptive capacity along elevation. Triangles and dots in Q2 represent hypothetical plant and bird species projected into a trait space, here exemplified for two dimensions. Polygons spanning the species in the trait space illustrate the functional diversity of the plant and the bird assemblage. Similar values of the functional diversity of plant and bird assemblages denote a high functional correspondence. Shown in Q3 are the projected vertical temperature shift, i.e., the projected upslope shift of a species' realized temperature niche, and the horizontal dispersal distance required to track the projected vertical temperature shift given the slope of the elevational gradient. To assess the plant species' climatetracking ability, I simulated plant-frugivore interactions and the plant species' longdistance dispersal (LDD) ability based on species' traits. 


\subsection{How do species' sensitivity to climate change and their adaptive capacity covary? (Q1)}

Species' potential responses to future climate change are often estimated with a focus on species' sensitivity to climate change (e.g., Thuiller et al. 2005, 2011; Bender et al. 2019). However, it is not yet clear whether the sensitivity of species to climate change is positively related to their adaptive capacity and consequently, whether the response of species to climate change can be estimated from studying exclusively species' sensitivity. To assess, how response traits and attributes related to the sensitivity of avian frugivore species to climate change and their adaptive capacity covary, I combined estimates of species' realized climatic niche breadth (approximating species' sensitivity to climate change) with response traits reflecting species' dispersal ability, dietary niche breadth and habitat niche breadth (i.e., aspects of species' adaptive capacity; Nowak et al. submitted, appendix A1). I hypothesized that (a) species' climatic niche breadth in frugivore assemblages increases with increasing elevation because increasing diurnal temperature variability at higher elevations should favour species with broad climatic niches (Ghalambor et al. 2006). In contrast, I expected that species' dispersal ability as well as their dietary and habitat niche breadth decrease with increasing elevation as the trait diversity of avian frugivores might be filtered by the environment at high elevations (Dehling et al. 2014b, a). Across species (b), I expected species' sensitivity to climate change and their adaptive capacity to be negatively related, because the sensitivity and the adaptive capacity of species that have survived climate change in the past could offset each other. This hypothesis is not clearly supported a priori.

\subsection{How will future climate change affect the functional correspondence of interacting species? (Q2)}

Climate change might lead to changes in the functional trait composition of the species assemblages (Pecl et al. 2017; Bender et al. 2019) and ultimately to a disruption of the functional correspondence between assemblages of interacting species. To assess how future climate change might affect the functional correspondence of fleshy-fruited plant and avian frugivore assemblages, I applied a new combination of future projections of species' elevational ranges with analyses of functional diversity (Nowak et al. 2019; appendix A2). I hypothesized that projected changes in the functional diversity of the avian frugivore assemblages might be particularly pronounced at mid- 
elevations because species turnover between current bird assemblages is high at midelevations (Jankowski et al. 2013b; Dehling et al. 2014a). I expected this to lead to a decoupling of the functional diversity of the projected fleshy-fruited plant and avian frugivore assemblages at mid-elevations.

\subsection{Is avian seed dispersal sufficient for plants to track future temperature change along elevation? (Q3)}

Recent range shifts of terrestrial plant species are reported to trail behind observed temperature shifts (Feeley et al. 2011b; Alexander et al. 2018; Lenoir et al. 2020), but quantitative estimates of plant species' climate-tracking ability are rare (but see Naoe et al. 2016; González-Varo et al. 2017). With a novel integration of two recently developed trait-based models, I simulated seed-dispersal distances avian frugivores can provide to fleshy-fruited plant species and quantified the number of long-distance dispersal events the plant species would require to fully track projected temperature shifts along elevation (Nowak et al. submitted, appendix A3). I hypothesized that (a) plant species require less long-distance dispersal events if the degree of trait matching is low as dispersal distances of bird-dispersed plants are larger in seed-dispersal systems with a low degree of trait matching (Sorensen et al. 2020). Furthermore, I hypothesized (b) that large-fruited plant species require fewer long-distance dispersal events than small-fruited plant species since large-fruited plant species interact preferably with large-billed, large-sized frugivores which provide long dispersal distances (Jordano et al. 2007; Wotton \& Kelly 2012; Burns 2013; Bender et al. 2018). The same may apply to canopy plants if plant height and fruit size are positively related (Shanahan \& Compton 2001; Thomson et al. 2011).

\section{Study system}

I studied impacts of climate change on interacting fleshy-fruited plant and avian frugivore species co-occurring along an elevational gradient in the Manú biosphere reserve located in the tropical Andes of southeast Peru ('Manú gradient' hereafter; Figure 2). The Manú gradient ranges from approximately 250 to $3750 \mathrm{~m}$ a.s.I.. The mean annual temperature along the Manú gradient decreases from about $24^{\circ} \mathrm{C}$ in the lowlands to about $7^{\circ} \mathrm{C}$ at the treeline (Girardin et al. 2010, 2013; Rapp \& Silman 2012). The mean annual precipitation is generally high, varying between $1500-4800 \mathrm{~mm}$ (mean = 2709 mm; Girardin et al. 2010, 2013; Rapp \& Silman 2012). The Manú 
gradient is entirely covered in rainforest. Four main forest types can be distinguished (Patterson et al. 1998): Lowland rainforest ( $\leq 500 \mathrm{~m}$ a.s.l.) is characterized by canopy trees up to $50 \mathrm{~m}$ high; Montane rainforest (about 500-1500 m a.s.I.) lacks such tall trees and has denser understory vegetation; Cloud forest (about 1500-2500 m a.s.I.) is intermittently covered in slowly rising clouds and is characterized by a high number of epiphytes and bryophyte layers on tree branches; Elfin forest (> $2500 \mathrm{~m}$ a.s.I.) comprises of compact, densely-branched trees and shrubs; At the treeline (about 3500 m a.s.I.), elfin forest gives way to Puna grassland (Patterson et al. 1998; Dehling et al. 2014b). The tropical Andes are one of the most biodiverse regions globally (Myers et al. 2000). The Manú biosphere reserve hosts about 1000 bird species (Walker et al. 2006). Similarly, it is a hotspot for plant diversity hosting alone several hundred tree species (Feeley et al. 2011b; Jankowski et al. 2013b).

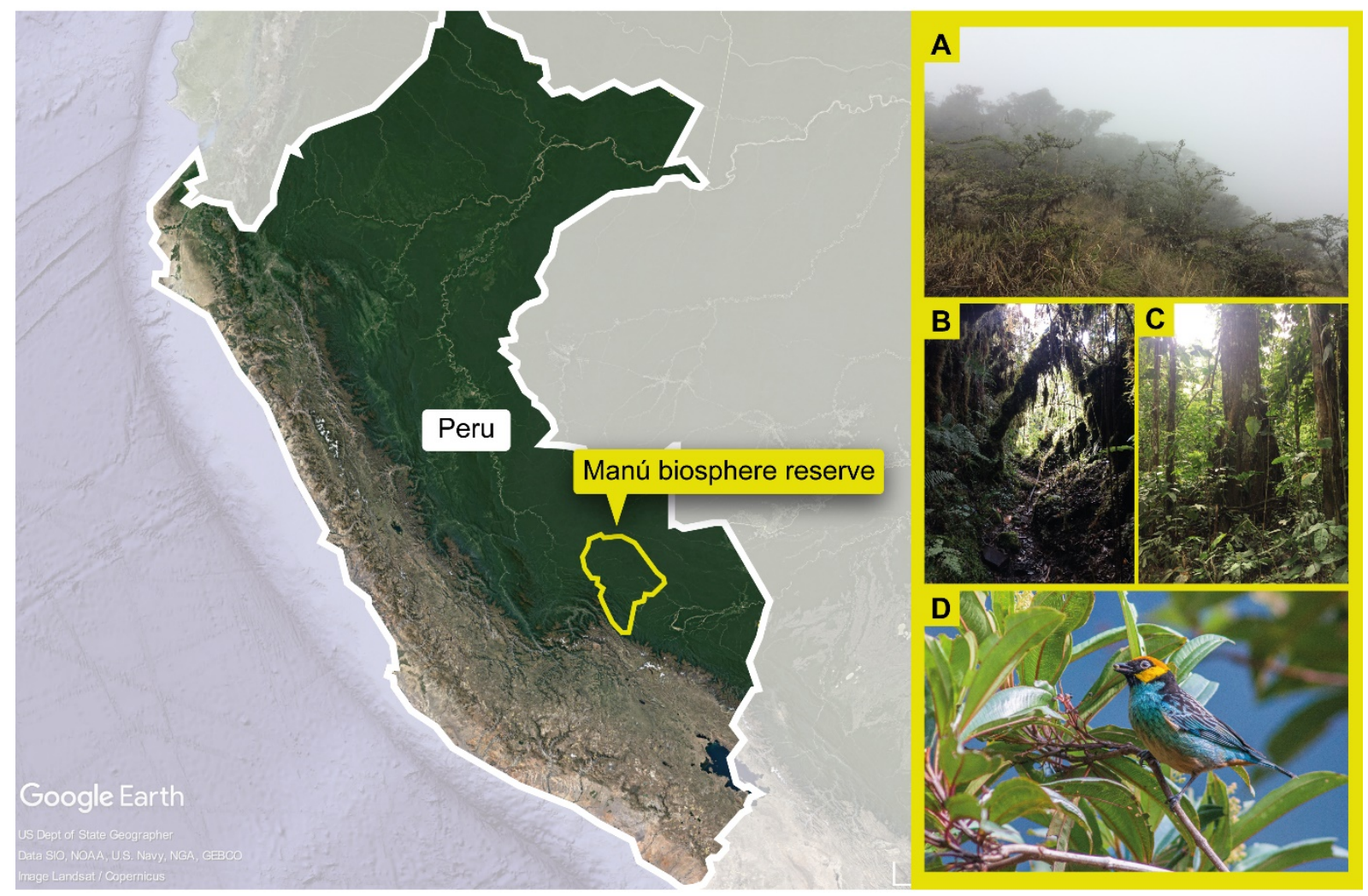

Figure 2: The Manú gradient is located in the Manú biosphere reserve in the Tropical Andes of southeast Peru. Shown are (A) elfin forest and patches of Puna grassland, (B) cloud forest, (C) lowland rainforest and (D) an avian frugivore (Tangara xanthocephala) feeding on a Miconia fruit (Melastomatacae). Authors of the pictures: (A-C) Larissa Nowak, (D) D. Matthias Dehling. Map adapted from Google Earth. 


\section{Data and methodological approaches}

\subsection{Elevational ranges of plants and birds}

I compiled elevational range data of 392 fleshy-fruited plant species and 245 avian frugivore species along the Manú gradient from literature. Fleshy-fruited plant species occurring along the Manú gradient were sampled and identified by Dehling et al. (2014b). Elevational distributions of these plant species in Peru were obtained from Brako and Zarucchi (1993) and supplemented with data from Tropicos (2017). Information on the elevational distributions of the avian frugivore species occurring along the Manú gradient was derived from local checklists (Walker et al. 2006; Merkord 2010), supplemented by field observations (Dehling et al. 2013). I excluded grounddwelling bird species $(n=18)$ from my analyses due to their distinct movement and foraging behaviour. Furthermore, I excluded the few migratory bird species that are not present year-round $(n=8)$. In my third chapter, I focussed on a subset of 343 woody fleshy-fruited plant species, as I was particularly interested in the climate-tracking ability of long-lived plant species.

\subsection{Trait data of plants and birds}

Morphological functional traits influence plant-bird interactions and avian seed dispersal (Jordano et al. 2007; Wotton \& Kelly 2012; Dehling et al. 2014b; Bender et al. 2018). I compiled morphological trait data of the fleshy-fruited plant species and the avian frugivore species from previous studies and literature. For the fleshy-fruited plant species, I compiled data on fruit width $(\mathrm{mm})$, fruit length $(\mathrm{mm})$, plant height $(\mathrm{m})$ and crop mass ( $g$; the fresh mass of a single fruit multiplied by the number of ripe fruits per plant). Fruit dimensions and fruit mass were measured on fresh fruits sampled during field surveys, while plant height and number of ripe fruits were estimated on plant individuals in the field (Dehling et al. 2014b; Bender et al. 2018). For the avian frugivore species, I compiled data on bill width $(\mathrm{mm})$, bill height $(\mathrm{mm})$, wing pointedness (Kipp's index or hand wing index sensu Sheard et al. 2020, measured as Kipp's distance/wing length) and body mass (g). Bill dimensions and wing shape were measured on museum specimens (Dehling et al. 2014a), following measurement protocols of Eck et al. (2011). I derived information on avian body mass from literature (Dunning 2007). 


\subsection{Sensitivity and adaptive capacity}

The sensitivity of species to climate change is influenced by their climatic niche breadth (IPCC 2007; Algar \& Tarr 2018; Foden et al. 2018). I estimated the realized climatic niche breadth of the avian frugivore species as two-dimensional hypervolume (Blonder et al. 2014) based on bioclimatic data and species' occurrences across South America. I obtained current bioclimatic data from the Climatologies at High resolution for the Earth's Land Surface Areas (CHELSA; Karger et al. 2017) and selected bioclimatic variables that capture minimum, maximum, mean, seasonality and diurnal variation of temperature and precipitation. Occurrence data for each bird species were downloaded from the Global Biodiversity Information Facility (GBIF.org 2017) and the data was subjected to a thorough quality check removing outliers and incorrect occurrence records (Bender et al. 2019; Nowak et al. submitted, appendix A1). Hypervolume estimates are most robust when computed in a low dimensionality (Blonder et al. 2014). Therefore, I performed a principal component analysis (PCA) across the bioclimatic variables at all occurrence points of the bird species in South America and used the first two axes of this PCA to compute each species' climatic niche breadth as two-dimensional hypervolume in the PCA space (Blonder et al. 2014).

The ability of species to track climate change and utilize novel resources are important aspects of their adaptive capacity (Dawson et al. 2011; Foden et al. 2018). This is determined by the species' dispersal ability and ecological niche breadth (Slatyer et al. 2013; Foden et al. 2018). I estimated the bird species' dispersal ability by their wing pointedness (i.e., hand-wing index sensu Sheard et al. 2020) which is positively related to their natal dispersal distances and their ability to fly over long distances (Winkler \& Leisler 1992; Dawideit et al. 2009; Santini et al. 2019). I approximated the bird species' dietary niche breadth by their bill width which influences the range of fruit sizes avian frugivores can feed on (Wheelwright 1985). I utilized data on species' habitat niche breadth from Barnagaud et al. (2017). In this dataset, the habitat niche breadth of the avian frugivore species was estimated as the number of habitat classes in which a species was recorded (Barnagaud et al. 2017). This was based on the habitat classification scheme (version 3) of the International Union for Conservation of Nature (IUCN). It included eleven habitat classes that represent a gradient from forest to open habitats. 


\subsection{Projecting the functional diversity of interacting species}

I projected the elevational ranges of the fleshy-fruited plant species and the avian frugivore species into the future considering three previously observed scenarios of how species' elevational ranges change under global warming (Freeman et al. 2018b, a). To this end, I computed the projected temperature increase along the Manú gradient based on current and future mean annual temperature downloaded from Wordclim (Hijmans et al. 2005). I then used the tropospheric lapse rate (i.e., the rate by which temperature decreases with increasing elevation; Mokhov \& Akperov 2006) to compute projected vertical distances (i.e., the distance by which a certain surface temperature shifts upslope under global warming) for the Manú gradient. Specifically, I computed the vertical distance at each elevation of the Manú gradient by dividing the projected temperature increase of an elevation by the tropospheric lapse-rate of that elevation. Finally, I computed species-specific vertical distances as the mean vertical distance across all elevations at which a species currently occurs. This estimates the distance a species has to shift its range vertically to track its current realized temperature niche under future global warming.

I applied three vertical dispersal scenarios, i.e., range contraction, range expansion and range shift. Under the range contraction scenario, I assumed that species cannot persist under temperatures that exceed their current realized temperature niche and that species are unable to shift upslope. Consequently, species' lower elevational range limit shifts upslope, but their upper elevational range limit does not change. Under the range expansion scenario, I assumed that species can tolerate or adapt to temperatures that exceed their current realized temperature niche and that species can shift upslope to track temperature shifts. Therefore, species' lower elevational range limit does not change, but their upper elevational range limit shifts upslope. Finally, under the range shift scenario, I assumed that species cannot persist under temperatures that exceed their current realized temperature niche but can shift upslope. As a result, species shift their lower and upper elevational range limits upslope and fully track their current realized temperature range along elevation.

I computed the multiple-trait functional diversity and the single-trait functional identity of the current and the projected fleshy-fruited plant and avian frugivore assemblages at the Manú gradient. Currently, the functional diversity and the functional identity of the fleshy-fruited plant and avian frugivore assemblages are positively related along the Manú gradient (Dehling et al. 2014b). I aimed to explore 
how future climate change might affect this functional correspondence between interacting species. For this, I considered morphological traits that have been shown to determine interactions between fleshy-fruited plants and avian frugivores via trait matching, i.e., the fruit dimensions and the bill dimensions, the plant height and the wing pointedness, as well as the crop mass and avian body mass (Dehling et al. 2014b; Bender et al. 2018). I computed the functional diversity of the plant and bird assemblages as functional richness. To this end, I computed Euclidean distances between the species of an assemblage according to their differences in the considered traits. I then performed a Principal Coordinate Analysis (PCoA) to project the species into a multidimensional trait space and calculated functional richness as the inner volume of a minimum convex hull spanning all species in the trait space (Villéger et al. 2008). In addition, I computed the functional identity of each plant and bird assemblage as the mean value of each trait across all species in an assemblage (Gagic et al. 2015). I assessed the functional correspondence of the fleshy-fruited plant and the avian frugivore assemblages under each vertical dispersal scenario. A significant positive relationship between the functional diversity and the functional identity of the plant and the bird assemblages along the Manú gradient denotes a high functional correspondence between the interacting assemblages.

\subsection{Trait-based simulations of plants' climate-tracking ability}

Both plant-frugivore interactions and seed-dispersal distances provided by avian frugivores are influenced by species' morphological traits (Jordano et al. 2007; Wotton \& Kelly 2012; Dehling et al. 2014b; Bender et al. 2018). Therefore, I integrated two recently developed trait-based simulation models to estimate plant species' longdistance dispersal ability and ultimately their ability to track future temperature change. First, I simulated interaction events between the woody fleshy-fruited plant species and the avian frugivores at the Manú gradient with a trait-based interaction model. This model is based on species' trait matching, particularly, the size difference between the bill width and the fruit width (Fründ et al. 2016; Donoso et al. 2017). In this model, the probability of an interaction is high if the size difference is zero (bill width = fruit width). A positive mismatch of the bill width and the fruit width (bill width $>$ fruit width) decreases the probability of an interaction. A negative mismatch (bill width $<$ fruit width) results in an interaction probability close to zero (Donoso et al. 2017) since small-billed birds cannot swallow fruits that are much wider than their bill (Bender et al. 2018). The 
degree of trait matching, i.e., how close the bill width and the fruit width need to match to make an interaction likely, can be adjusted in the model. I simulated interaction events considering a low, an intermediate and a high degree of trait matching corresponding to a low, an intermediate and a high degree of specialization of the seed-dispersal interactions. Second, I applied a trait-based seed-dispersal model to simulate plant species' long-distance dispersal ability (Sorensen et al. 2020). Specifically, I computed a seed-dispersal distance for each simulated interaction event based on the body mass of the avian disperser and allometric scaling of avian gut passage time and flight speed (Sorensen et al. 2020). I then approximated the longdistance dispersal ability of the bird-dispersed plant species as the $95 \%$ quantile of all simulated dispersal distances per plant species (Donoso et al. 2020). Finally, to assess the climate-tracking ability of the plant species, I computed the number of long-distance dispersal events the plant species would require to fully track projected upslope shifts of their realized temperature niche under future climate change. Specifically, I compared a plant species' long-distance dispersal ability to the horizontal dispersal distance required to track the projected vertical temperature shift given the average slope of the Manú gradient.

\section{Main results and discussion}

\subsection{Independent variation of avian sensitivity to climate change and adaptive capacity (Q1)}

I assessed relationships between response traits and attributes related to the sensitivity of avian frugivore species to climate change and their adaptive capacity (Nowak et al. submitted, appendix A1). I found that species' climatic niche breadth increased with increasing elevation. Contrastingly, their dispersal ability, approximated by their wing pointedness, and their dietary niche breadth, approximated by their bill width, decreased with increasing elevation. Across species, there was no significant relationship of response traits and attributes related to the sensitivity of the avian frugivores to climate change and their adaptive capacity. The independent variation of species' sensitivity and adaptive capacity suggests that reliable future projections of species assemblages under climate change require consideration of species' sensitivity and their adaptive capacity.

The positive relationship of species' climatic niche breadth with elevation was according to my expectations. This relationship is in line with Rapoport's rule stating 
that species' range sizes increase with increasing elevation due to a greater tolerance for climate variation of species at high elevations (Stevens 1989, 1992). It is further consistent with Janzen's climate variability hypothesis according to which species from more variable climates have broader thermal niches than species from stable climates (Janzen 1967; Ghalambor et al. 2006). This relationship has also been confirmed for bird species along latitudinal gradients (Khaliq et al. 2014). Along the Manú gradient, the diurnal temperature variation increases with increasing elevation, which might favour avian frugivore species with larger climatic niches in high-elevation assemblages (Ghalambor et al. 2006; Rapp \& Silman 2012). Accordingly, it has been reported that bird species from high elevations in the Peruvian Andes are more resistant to heat loss, but as capable of tolerating high lowland temperatures as species from lower elevations (Londoño et al. 2017).

The negative relationships of wing pointedness and bill width with elevation were according to my expectations. Since most avian frugivores in the tropics highly depend on fruit in their diet (Kissling et al. 2009), these relationships might be due to bottomup effects of the fleshy-fruited plant assemblages on the trait composition of the avian frugivore assemblages (Githiru et al. 2002; Vollstädt et al. 2017). Along the Manú gradient, low plant heights at high elevations might favour rounded-winged bird species at these elevations, due to trait matching between the plant height and avian wing pointedness (Dehling et al. 2014b; Bender et al. 2018). Similarly, small fruit sizes at high elevations might promote small-billed frugivores in high-elevation assemblages, due to trait matching between the fruit width and the bill width (Burns 2013; Dehling et al. 2014b; Bender et al. 2018). Moreover, the availability of resources decreases with increasing elevation, which may cause environmental filtering of bird traits at high elevations, resulting in a lower trait diversity of high-elevation bird assemblages (Hanz et al. 2019).

The opposite patterns of species' sensitivity to climate change and their adaptive capacity along elevation imply that avian frugivore species from low and high elevations might respond differently to climate change. Their rather narrow climatic niche suggests that the avian frugivore species from lowland assemblages might be sensitive to climate change and shift their elevational ranges upslope to track their suitable climate under contemporary climate change. Indeed, bird species along tropical elevational gradients have been reported to shift their ranges upslope under contemporary climate change (Forero-Medina et al. 2011; Freeman \& Class Freeman 
2014; Freeman et al. 2018b). Due to their good dispersal ability, pointed-winged species of the lowland assemblages might be especially well equipped to shift their ranges upslope (Dawideit et al. 2009; Santini et al. 2019). Moreover, broad-billed lowland species might be well able to find matching fruit resources at higher elevations, because broad-billed bird species are able to feed on a wide range of fruit sizes, thus being flexible in their fruit choice (Wheelwright 1985). Yet, changes in the fruit resource use of avian frugivores have thus far undergone little research (Bender et al. 2017).

Avian frugivore species from assemblages at higher elevations might be less sensitive to climate change, due to their rather broad climatic niches. In line with this, avian frugivore species at high elevations in the tropics were projected to persist in their current assemblages despite future climate change (Bender et al. 2019). Furthermore, globally, plant and animal species from high elevations have shifted their ranges upslope at lower rates than species from low elevations in response to contemporary climate change, potentially due to their broader climatic niches (Mamantov et al. 2021). However, avian frugivore species from high-elevation assemblages might be less well equipped to adjust to changes in the fruit resource availability. Due to their rather narrow bills, many species are restricted to feed on small fruits (Wheelwright 1985). Similarly, due to their round wings, many species are better adapted to forage efficiently in low than in high forest strata (Howe \& Smallwood 1982; Dehling et al. 2014b). Currently, tropical plant species are shifting their ranges towards higher elevations in response to contemporary climate change (Feeley et al. 2011b, a; Morueta-Holme et al. 2015). Projections suggest that upslope range shifts of largefruited and tall plant species from tropical lowlands can increase the average fruit size and plant height of assemblages of fleshy-fruited plants at higher elevations (Nowak et al. 2019, appendix A2). Thus, avian frugivore species that currently occur at high elevations might not be able to efficiently forage on the fruit resources provided by the future plant assemblages at high elevations (Fleming 1979; Dehling et al. 2014b).

Contrary to my expectations, species' response traits and attributes related to species' sensitivity to climate change and adaptive capacity were unrelated across the avian frugivores. Indeed, I found that species' wing pointedness, bill width and habitat niche breadth varied widely across the entire variation of species' climatic niche breadths. Coinciding with this independence between species' sensitivity and adaptive capacity, animal species globally have been reported to differ strongly in their responses to climate change, for example, in the magnitude of their range shifts (Moritz 
et al. 2008; Chen et al. 2011; Lenoir et al. 2020). My results show that even within the ecologically homogenous guild of avian frugivores species, responses to climate change will likely be idiosyncratic.

Previous studies found varying relationships of species' sensitivity and aspects of their adaptive capacity across species. For example, the habitat niche breadth of European bird species has been reported to be positively related to their dietary niche breadth and negatively related to their climatic niche breadth (Reif et al. 2016). Contrastingly, habitat niche breadth and climatic niche breadth were positively related in a study focussing on French bird species (Barnagaud et al. 2012). My results confirm neither of these relationships. This suggests that the relationship of sensitivity and adaptive capacity across species may be context-dependent. Specifically, the relationship seems to differ between functionally diverse assemblages of tropical frugivore species, studied here, and the functionally less diverse European bird assemblages that include migratory species studied earlier.

\subsection{Low functional correspondence of interacting species under a scenario of range contraction (Q2)}

I applied a new combination of future projections of species' elevational ranges with analyses of functional diversity to simulate how different scenarios of temperaturedriven range changes may affect the functional correspondence of fleshy-fruited plants and avian frugivores (Nowak et al. 2019, appendix A2). The functional correspondence of fleshy-fruited plants and avian frugivores was lowest if plant and bird species were projected to contract their ranges towards higher elevations in response to increasing temperatures. Contrastingly, if species were projected to expand their ranges upslope, the functional correspondence remained high. This shows that the integrity of biotic interactions under climate change depends on species' upslope range shifts.

The low functional correspondence under a scenario of range contraction was due to a stronger projected decline of bird than plant functional diversity and a projected mismatch in mean trait values of the plant and bird assemblages at midelevations of the Manú gradient. Currently, there is a strong taxonomic and functional turn over between avian frugivore assemblages at mid-elevations of the Manú gradient, i.e., the neighbouring frugivore assemblages differ most in their species composition and functional diversity at mid elevations (Jankowski et al. 2013b; Dehling et al. 2014a). This might explain why range contraction has a particularly strong effect 
on mid-elevation frugivore assemblages. It has previously been proposed that large proportions of montane bird species globally are threatened by climate change if species are limited in their upslope dispersal but sensitive to increasing temperatures (La Sorte \& Jetz 2010). Similarly, observed range contractions of montane species under contemporary climate change were accompanied by population declines and erosion of genetic diversity (Rubidge et al. 2012; Freeman et al. 2018a, b). My results go beyond this in suggesting that limited upslope dispersal under climate change not only may negatively affect individual species, but may also disrupt key biotic interactions between species.

The low functional correspondence of fleshy-fruited plant and avian frugivore assemblages under range contraction indicates that plant species with specific sets of functional traits might miss matching avian seed dispersers in future species assemblages (Burns 2013; Dehling et al. 2014b; Bender et al. 2018). For example, I found a projected mismatch between the average fruit size and the average bill size at mid-elevations under range contraction, mostly due to a projected reduction of the average bill size in the frugivore assemblages. This indicates that the seed dispersal of large-fruited plant species might be reduced in future assemblages due to a loss of large-billed frugivores. Indeed, a loss of large-billed, large-bodied frugivores due to hunting and logging has been shown to reduce the dispersal of large-fruited plant species (Markl et al. 2012). At high elevations, my projections further revealed an increase of the average bill width, while the average fruit width was projected to change little under range contraction. However, this mismatch of plant and bird traits might have less drastic consequences because broad-billed avian frugivores are more flexible in their fruit choice (Wheelwright 1985). The flexibility of avian frugivores to change to other fruit resources might reduce the negative impacts of climate change on avian seed dispersal (Bender et al. 2017). Nevertheless, eventually, a mismatch between plant and bird traits could cause evolutionary changes in plant assemblages, for instance, with negative consequences for large-fruited plant species (Galetti et al. 2013; Onstein et al. 2018; Sales et al. 2021a).

The high functional correspondence between plant and avian frugivore assemblages under a scenario of range expansion suggests that if species are able to shift their upper elevational range limits upslope, a loss of seed dispersal functions under climate change is less likely. Similarly, montane bird species globally were projected to be less negatively affected by climate change, if species can shift their 
upper elevational range limit upslope (La Sorte \& Jetz 2010). A tendency for range expansions under contemporary climate change has indeed been observed for lowland plant and animal species from elevational gradients globally (Freeman et al. 2018a, b).

\subsection{Avian seed dispersal might be insufficient for plants to track future temperature change along elevation (Q3)}

I applied a novel combination of trait-based simulation models and temperature projections to quantify the ability of bird-dispersed woody plant species to track projected vertical temperature shifts along a tropical elevational gradient (Nowak et al. submitted, appendix A3). I found that most plant species required several longdistance dispersal events to fully track the projected vertical temperature shifts in time, even under low and intermediate projected temperature increase. However, I also found that the number of required long-distance dispersal events varied strongly with the degree of trait matching and with plant species' traits.

The results of my simulations suggest that avian seed dispersal is insufficient for plants to track future temperature change along the elevational gradient. In my study, I considered a fixed time window until 2070 and quantified the number of long-distance dispersal events plant species would require to fully track projected vertical temperature shifts within this time window. According to my simulations, most plant species require several long-distance dispersal events. However, long-lived woody plant species might not be able to undergo several consecutive long-distance dispersal events within such a short time window because long times to maturity and long generation times can slow down their dispersal ability over time (Skellam 1951; Salguero-Gómez et al. 2015, 2016; Lustenhouwer et al. 2017). In line with this, recent upslope range shifts of tropical tree genera have been reported to lag behind vertical temperature shifts under contemporary climate change (Feeley et al. 2011b). It has been shown previously that seed dispersal mediated by mammals can help plants to track shifts in their suitable climate along elevation (Naoe et al. 2016; González-Varo et al. 2017). Contrastingly, my simulations suggest that seed dispersal mediated by avian frugivores might not be sufficient for plants to track vertical temperature shifts under future climate change.

As expected, my simulations revealed that plant species in seed dispersal systems with a low degree of trait matching required fewer long-distance dispersal events to track projected vertical temperature shifts than plant species from seed 
dispersal systems with a high degree of trait matching. This indicates that the resilience of plant species to future climate change may depend on the type of seed dispersal system. In systems with a low degree of trait matching, large-billed and large-bodied avian frugivore species feed on a wider range of plant species (Sorensen et al. 2020). Therefore, small-fruited plant species interact with a greater diversity of seed dispersers, which increases their chance to be dispersed by large disperser species that transport seeds over long distances (Jordano et al. 2007; Wotton \& Kelly 2012; Sorensen et al. 2020). This is in line with the theory of a sampling effect of biodiversity, according to which diversity increases ecosystem processes and functions by increasing the chance that species with relevant functional traits are present (Tilman 2001). Previous studies showed that seed dispersal systems tend to be less specialized at low than at high latitudes and in the Afrotropics compared to the Neotropics (Schleuning et al. 2012; Dalsgaard et al. 2017; Dugger et al. 2019), while the degree of trait matching is positively related to the degree of specialization in seed dispersal systems (Sorensen et al. 2020). Moreover, the degree of specialization of seed dispersal systems can increase with increasing elevation and tends to be higher in the forest interior compared to forest edges (Menke et al. 2012; Quitián et al. 2018). Consequently, the ability of bird-dispersed plant species to track climate change might vary considerably at global and local scales depending on the type of seed-dispersal system.

A low degree of specialization is usually viewed as advantageous for species under environmental change (Gallagher et al. 2015). However, the focus so far has lied upon species' diet or habitat specialization, while the effect of biotic specialization on species interactions under environmental change are less well studied (Heikkinen et al. 2010; Culp et al. 2017; MacLean \& Beissinger 2017). It has been shown that a low degree of specialization in biotic interactions can lower the risk of extinction cascades driven by the loss of interaction partners (Aizen et al. 2012; Brodie et al. 2014; Correa et al. 2016). My results suggest that the specialization of seed-dispersal interactions may also reduce the capability of plants to track shifts in their suitable climate.

In line with my expectations, my simulations revealed that large-fruited and canopy plant species required fewer long-distance dispersal events to track projected vertical temperature shifts than small-fruited and understory plant species. I found this independent of the degree of trait matching. Consequently, large-fruited and canopy 
plant species may be better able to track projected vertical temperature shifts than small-fruited and understory plant species. This is because large-billed, large-sized avian frugivore species preferably interact with large-fruited canopy plant species and disperse their seeds over long distances (Jordano et al. 2007; Wotton \& Kelly 2012; Burns 2013; Dehling et al. 2014b; Bender et al. 2018). Consistent with these results, fossil pollen data shows that large-seeded plant assemblages have better tracked climate change during the Late Quaternary than small-seeded plant assemblages (Knight et al. 2020). Likewise, it has been reported that large-seeded understory plant species better track contemporary climate change compared to small-seeded species from the understory (Ash et al. 2017).

\section{Synthesis and conclusions}

\subsection{Trait-based approaches to study the impacts of climate change on interacting species}

Trait-based approaches are promising tools to study the impacts of climate change on biodiversity. On the one hand, trait data are becoming increasingly available via new measurements, the publication of data papers and open-access databases (Kissling et al. 2018; Kattge \& et al. 2020; Sheard et al. 2020). On the other hand, species' traits are ecologically relevant, influencing how species respond to and affect their abiotic and biotic environment (Tilman 2001; Violle et al. 2007; Suding et al. 2008; Schleuning et al. 2020). Therefore, trait-based approaches offer exciting possibilities in globalchange research.

In this dissertation, I explored how trait-based approaches can increase understanding of the impacts of future climate change on interacting species. This work builds upon empirical measurements of key morphological functional traits of large numbers of fleshy-fruited plant and avian frugivore species. I showed that functional response traits related to species' sensitivity to climate change and their adaptive capacity should be considered together to project species assemblages under climate change reliably (Nowak et al. submitted, appendix A1). Furthermore, I showed how functional matching traits can help to identify ecological systems that might be prone to a functional disruption of biotic interactions under climate change (Nowak et al. 2019, appendix A2). Finally, I showed how plant species' ability to track climate change can be estimated with functional matching traits and functional response traits related to avian seed dispersal (Nowak et al. submitted, appendix A3). 
The trait-based approaches employed and developed further in my dissertation are applicable more widely. First, they may be applied to other geographic regions. For example, trait-based simulations of avian seed dispersal can be applied to assess the potential of avian dispersers to contribute to the climate-tracking ability of birddispersed plants in various seed dispersal systems globally, including Afrotropic, Mediterranean or temperate seed dispersal systems (González-Castro et al. 2012; Plein et al. 2013; Dugger et al. 2019). Trait-based simulations can be applied to assess the plant species' ability for vertical and lateral range shifts. Similarly, the impacts of climate change on the functional correspondence of interacting species may be assessed in other ecosystems globally.

Second, these trait-based approaches allow assessing the impacts of climate change on other types of biotic interactions. For instance, projections of the functional diversity of interacting species enable detecting a potential functional disruption of other trophic interactions that are influenced by trait matching. This applies, for example, to mutualistic plant-pollinator interactions (Stang et al. 2009; Maglianesi et al. 2014; Weinstein \& Graham 2017). Similarly, trait matching influences antagonistic interactions, such as plant-herbivore and predator-prey interactions (Bartomeus et al. 2016). Relevant matching traits in plant-herbivore interactions are, for instance, the insects' incisive strength and the plants' leaf dry matter content or the plants' chemical defences (Jamieson et al. 2017; Le Provost et al. 2017). Examples of matching traits in predator-prey interactions are the predator-prey body size ratio as well as the predator's bite force and the prey's cuticular toughness (Gravel et al. 2013; Brousseau et al. 2018). Identifying such functional matching traits will allow projecting the functional diversity of interacting species and detecting potential spatial and temporal mismatches in different types of biotic interactions.

Third, these trait-based approaches may be applied to assess the impacts of other drivers of global change on interacting species. For instance, trait-based simulations of the dispersal ability of bird-dispersed plants can be applied to assess plant species' ability to track both shifts in their suitable climate and habitat which often occur jointly (Hof et al. 2018; Newbold 2018). Furthermore, projections of the functional diversity of interacting species may be applied to explore how other aspects of global change, such as land-use change, defaunation and species invasions, as well as their combined effects may alter the functional correspondence of interacting species 
(Lambin et al. 2003; Strayer et al. 2006; Dirzo et al. 2014; Seebens et al. 2015; Hof et al. 2018; Newbold 2018).

Finally, the trait-based approaches employed in my work can be further developed. Different drivers of global change are causing species to migrate into and out of species assemblages, or become extinct from them. This process happens over time, and some changes may occur gradually, while others might happen abruptly (Trisos et al. 2020). Consequently, to improve biodiversity projections under global change, we might need to move towards a dynamic modelling framework to capture changes in species assemblages over time (Miele \& Matias 2017). Combining traitbased projection models of species occurrences with trait-based models of interaction rewiring might help to achieve this. Specifically, in a first step, species' potential future distributions under global change could be projected under consideration of species' functional response traits. For bird-dispersed plant species, trait-based simulations of the plant species' dispersal ability may be integrated into projection models of plant species' future distributions under global change (Di Musciano et al. 2020). In a second step, rewiring models could be applied to simulate which species might interact with each other in the reshuffled species assemblages (Vizentin-Bugoni et al. 2020). Rewiring models allow to compute interaction probabilities between species based on species' co-occurrences and the compatibility of their functional matching traits (Pichler et al. 2020; Vizentin-Bugoni et al. 2020). This allows estimating which species have a high risk to go secondarily extinct due to a lack of potential interaction partners with matching traits in reshuffled species assemblages. Finally, these two modelling steps, i.e., future projections of species occurrences and simulations of interaction rewiring, might be implemented for several consecutive time steps at a high temporal resolution to account for the dynamic component of biodiversity under global change.

\subsection{Climate change and interacting species along tropical elevational gradients}

Trait-based approaches can foster an understanding of how interacting species from different assemblages along environmental gradients may respond to climate change. My results and previous studies suggest that species assemblages from different elevations in the tropics differ in their trait composition and consequently likely also in their responses to climate change (Dehling et al. 2014a, b). Specifically, in the tropical lowlands, species assemblages tend to host species with rather narrow climatic niches. This has been found for animal species including birds and according to the climate 
variability hypothesis, it may also apply to plants (Janzen 1967; Ghalambor et al. 2006; Shah et al. 2017; Fang et al. 2019, Nowak et al. submitted, appendix A1). Yet, lowland assemblages in the tropics also host plant and bird species whose traits suggest a good dispersal and climate-tracking ability (Dehling et al. 2014a, b; Nowak et al. 2019, appendix A2, Nowak et al. submitted, appendix A1). In contrast, tropical high-elevation assemblages comprise species with broader climatic niches but traits that suggest a lower dispersal and climate-tracking ability (Janzen 1967; Ghalambor et al. 2006; Dehling et al. 2014a, b; Shah et al. 2017; Fang et al. 2019; Nowak et al. 2019, appendix A2, Nowak et al. submitted, appendix A1).

Based on my and previous findings, we might expect that the sensitive, but mobile lowland species shift their lower and upper elevational range limits upslope to track their suitable climate under climate change. However, thus far, lowland species tend to expand their elevational ranges towards higher elevations under climate change (Freeman et al. 2018a, b). Hence, contemporary climate change might not have exceeded the climatic niche of lowland species yet. On the contrary, we might expect the less sensitive species from high elevations to persist in their current elevational ranges under climate change. However, empirical evidence suggest that highelevation species are contracting their ranges towards the mountain top (Freeman et al. 2018a, b). Hence, lower elevational range limits of high-elevation species might be pushed upslope by competition with immigrating species from lower elevations or by other biotic factors, such as interactions with predators or pathogens (Jankowski et al. 2010, 2013a).

Disentangling such discrepancies between projections and observations might be an interesting challenge for future studies. One way to address this could be comparisons between projections and empirical observations. Such comparisons may allow testing whether observed patterns of species' range shifts under climate change can be predicted more closely when including physiological data or accounting for mutualistic and antagonistic interactions (Jankowski et al. 2013a). Furthermore, more comparisons between geographic regions would be desirable. For instance, recent meta analyses on observed elevational range shifts of species globally are largely biased towards temperate datasets (Freeman et al. 2018a; Mamantov et al. 2021). Hence, more empirical data on other tropical regions might facilitate our understanding of geographic differences in the effects of climate change on species assemblages along elevational gradients. 
Some conservation implications for interacting plant and bird species along tropical elevational gradients may be derived from my and previous findings. First, it is important to enabling species to shift their ranges upslope. This is relevant to allow species tracking their suitable climate and protect them from range contractions. Moreover, the results of my dissertation suggest that it may help in preventing a functional disruption of interacting species under range contraction (Nowak et al. 2019, appendix A2). To achieve this, avoiding deforestation and land-use change on tropical mountains is crucial (Sales et al. 2019). Furthermore, planning for movement corridors between habitat fragments can increase the connectivity of an area and facilitate dispersal of plants and animals (Tewksbury et al. 2002). Second, for animal-dispersed organisms, it is crucial to protect their seed dispersers. Protecting large disperser species is thereby particularly important, as they mediate long-distance dispersal (Westcott \& Graham 2000; Bascompte \& Jordano 2007). Furthermore, large disperser species are essential for the dispersal and climate-tracking ability of large-fruited plant species (Galetti et al. 2013; Bender et al. 2018). To protect large disperser species, drivers of defaunation need to be reduced. Besides climate change itself, these include overexploitation, habitat destruction and species invasions (Dirzo et al. 2014; Bogoni et al. 2020). In addition, the rewilding of large dispersers might be an option to restore the provisioning of long-distance seed dispersal (Svenning et al. 2016; Galetti et al. 2017). Third, it would be important to avoid additional pressure from land-use change, especially for species assemblages at the lowest and highest elevations of tropical mountains. At low elevations, land-use change might accelerate lowland biotic attrition (Guo et al. 2018). At high elevations, it may put additional pressure on high-elevation species whose upslope range shifts are already limited by the mountain top (Freeman et al. 2018b). Finally, it is relevant to limit global warming (IPCC 2018). The results of my dissertation suggest that limiting global warming, e.g., to well below $2{ }^{\circ} \mathrm{C}$ as agreed upon in the Paris Agreement, reduces the risk that species' range shifts lag behind shifts in their suitable climate (Nowak et al. submitted, appendix A3; IPCC 2018). This will be particularly relevant for poor dispersers and long-lived species (Nowak et al. submitted, appendix A3; Lenoir et al. 2020). In addition, limiting global warming may protect high-elevation species from being pushed towards the mountain top (Freeman et al. 2018b).

Considering biotic interactions is important for both projecting biodiversity and targeting conservation efforts under global change (Tylianakis et al. 2010; Heinen et 
al. 2020; Schleuning et al. 2020). Accounting for biotic interactions in conservation programmes can foster the maintenance of self-sustaining ecosystems, as intact biotic interactions are crucial for the interacting species and ecosystem functioning alike (Tylianakis et al. 2010; Schleuning et al. 2015; Heinen et al. 2020). For example, not only do intact plant-frugivore interactions ensure the nutrition of the frugivores and the seed dispersal of the plants, but they are also important for the long-term stability and the natural regeneration of forests (Wunderle Jr 1997; Wang \& Smith 2002; Norden et al. 2009; Jordano 2014; Carlo \& Morales 2016). To conserve intact biotic interactions and their associated ecosystem functions, we need to understand how the interacting species might be impacted by ongoing global change. In this dissertation, I showed how a trait-based perspective can help to gain such knowledge about the future of biodiversity. 


\section{Zusammenfassung}

Biologische Vielfalt ist unabdingbar für die Funktionsfähigkeit von Ökosystemen und für das Wohlergehen der Menschheit. Trotzdem verändert sich die biologische Vielfalt der Erde schnell. Ein wichtiger Treiber dieser Veränderung ist der Klimawandel. Seit der vorindustriellen Zeit (1850-1900) ist die globale Temperatur um etwa $1{ }^{\circ} \mathrm{C}$ angestiegen. Dies hat unter anderem zur Folge, dass überall auf der Erde Arten ihre Verbreitungsgebiete in Richtung der Pole oder in größere Höhenlagen verschieben. Diese Entwicklung verändert die Zusammensetzung von Artgemeinschaften weltweit. Um angesichts des voranschreitenden Klimawandels Politik und Naturschutz zu informieren bedarf es zuverlässiger Projektionen der biologischen Vielfalt.

Die Arten auf der Erde sind in vielfältige biotische Wechselbeziehungen verstrickt. Die miteinander interagierenden Arten können unterschiedlich auf den Klimawandel reagieren. Dies kann zur Folge haben, dass das räumliche oder zeitliche Vorkommen der ehemals interagierenden Arten nicht mehr übereinstimmt, was negative Konsequenzen für die Arten nach sich ziehen kann. Verliert eine Art beispielsweise einen notwendigen Interaktionspartner, kann die Art sekundär aussterben. Daher ist es wichtig, biotische Wechselbeziehungen in Projektionen der biologischen Vielfalt einzubeziehen.

Der Einfluss des Klimawandels auf die biologische Vielfalt wird häufig mittels der Sensitivität der Arten, d.h. mittels deren Klimanische, abgeschätzt. Ein weiterer Ansatz sind sogenannte Verwundbarkeitsanalysen, die die Sensitivität der Arten gemeinsam mit deren Anpassungsfähigkeit, z. B. deren Ausbreitungsfähigkeit oder ökologischer Flexibilität, bewerten. Diese Ansätze liefern wichtige Einblicke, doch sie haben auch Schwachpunkte. Es ist beispielsweise nicht klar, wie die Sensitivität von Arten gegenüber dem Klimawandel und ihre Anpassungsfähigkeit in Beziehung stehen. Ein Fokus auf die Sensitivität der Arten könnte daher ein unvollständiges Bild darüber liefern, wie Arten auf den Klimawandel reagieren. Verwundbarkeitsanalysen ziehen zwar sowohl die Sensitivität, als auch die Anpassungsfähigkeit der Arten in Betracht, doch diese Informationen werden nicht in Projektionen der biologischen Vielfalt eingebunden. Darüber hinaus berücksichtigen beide Ansätze die Rolle biotischer Wechselbeziehungen bislang nur unzureichend.

Der Einfluss des Klimawandels auf interagierende Arten wird von funktionellen Merkmalen der Arten beeinflusst. Funktionelle Merkmale sind messbare Eigenschaften der Arten, die die abiotischen und biotischen Wechselbeziehungen der 
Art beeinflussen. Die Sensitivität und die Anpassungsfähigkeit der Arten werden von funktionellen Reaktionsmerkmalen beeinflusst. Das sind Merkmale, die bestimmen, wie Arten auf ihre Umwelt reagieren. Eine Analyse funktioneller Reaktionsmerkmale kann unser Verständnis darüber verbessern, wie Arten auf den Klimawandel reagieren.

Funktionelle Übereinstimmungsmerkmale hingegen beeinflussen, ob Arten in der Lage sind, in durch den Klimawandel veränderten Artgemeinschaften kompatible Interaktionspartner zu finden. Das Übereinstimmen dieser Merkmale zwischen zwei Arten bestimmt, ob die beiden Arten eine Wechselbeziehung eingehen können. Die Vielfalt dieser funktionellen Übereinstimmungsmerkmale deckt sich in der Regel in Lebensgemeinschaften interagierender Arten. Eine Analyse funktioneller Übereinstimmungsmerkmale erlaubt es daher abzuschätzen, ob diese funktionelle Entsprechung in den Lebensgemeinschaften durch den Klimawandel gestört werden könnte. Die Analyse funktioneller Merkmale der Arten eröffnet vielversprechende, aber bislang wenig genutzte Möglichkeiten, zu erkunden, wie sich der Klimawandel auf interagierende Arten auswirkt. Ich konzentriere mich in meiner Dissertation auf den Einfluss des Klimawandels auf Wechselbeziehungen zwischen Fruchtpflanzen und fruchtfressenden Vögeln entlang eines tropischen Höhengradienten.

Im ersten Kapitel meiner Dissertation beschäftigte ich mich mit der Frage, wie die Sensitivität der fruchtfressenden Vögel gegenüber dem Klimawandel und ihre Anpassungsfähigkeit miteinander variieren. Zu diesem Zweck kombinierte ich Schätzungen der Klimanischenbreite von Arten mit funktionellen Reaktionsmerkmalen, die die Ausbreitungsfähigkeit der Arten sowie ihre Nahrungsnischenbreite und ihre Lebensraumnischenbreite abbilden. Ich erwartete, dass die Klimanischenbreite in Artgemeinschaften fruchtfressender Vögel mit zunehmender Höhe entlang des Höhengradienten zunehmen würde, da die zunehmende tageszeitliche Temperaturvariabilität Arten mit größerer Klimanische in den Hochlagen bevorzugen sollte. Andererseits erwartete ich, dass die Ausbreitungsfähigkeit, sowie die Nahrungs- und die Lebensraumnischenbreite der Arten mit zunehmender Höhe abnehmen sollten, bedingt durch den systematischen Verlust bestimmter funktioneller Merkmale von fruchtfressenden Vögeln in höheren Lagen. Über die Arten hinweg erwartete ich, dass Aspekte der Sensitivität und der Anpassungsfähigkeit negativ zusammen hängen sollten, da dies die langfristige Überlebensfähigkeit von Arten in der Evolutionsgeschichte erhöhen sollte. Meine 
Analysen ergaben in der Tat, dass die Klimanischenbreite der Arten mit zunehmender Höhe zunahm, während die Ausbreitungsfähigkeit und die Nahrungsnischenbreite mit zunehmender Höhe abnahmen. Die gegensätzlichen Beziehungen der Sensitivität der Arten und Aspekten ihrer Anpassungsfähigkeit mit der Höhe deuten darauf hin, dass fruchtfressende Vogelarten aus dem Tiefland womöglich anders auf den Klimawandel reagieren als Arten aus größeren Höhen. Durch ihre eher kleinen Klimanischen sind Tieflandarten möglicherweise empfindlich gegenüber dem Klimawandel und müssen ihre Verbreitungsgebiete in höhere Lagen verschieben. Tieflandarten mit einer guten Ausbreitungsfähigkeit scheinen dabei besonders gut für solche Verbreitungsverschiebungen ausgestattet zu sein. Gleichermaßen sollten Tieflandarten mit einer eher breiten Nahrungsnische gut in der Lage sein, neue Fruchtressourcen in höheren Lagen zu erschließen. Fruchtfressende Vogelarten aus dem Hochland hingegen, sollten durch ihre eher breite Klimanische weniger empfindlich auf den Klimawandel reagieren. Allerdings sind Hochlandarten durch ihre eher enge Nahrungsnische vermutlich weniger gut an mögliche klimabedingte Veränderungen in der Verfügbarkeit von Fruchtressourcen angepasst. Über die Arten hinweg ergaben meine Analysen, dass Aspekte der Sensitivität und der Anpassungsfähigkeit der fruchtfressenden Vögel voneinander unabhängig sind. Dies war entgegen meiner Erwartungen und deutet an, dass sich einzelne Arten in ihren Reaktionen auf den Klimawandel unterscheiden. Zusammen genommen deuten meine Ergebnisse darauf hin, dass es wichtig ist, sowohl die Sensitivität der Arten gegenüber dem Klimawandel als auch ihre Anpassungsfähigkeit zu berücksichtigen, wenn man den möglichen Einfluss des Klimawandels auf die biologische Vielfalt ganzheitlich verstehen möchte.

Im zweiten Kapitel meiner Dissertation untersuchte ich, wie der voranschreitende Klimawandel die funktionelle Entsprechung von Lebensgemeinschaften interagierender Fruchtpflanzen- und fruchtfressender Vogelarten beeinflussen könnte. Zu diesem Zweck kombinierte ich Zukunftsprojektionen der Höhenverbreitung der Arten mit Analysen der Vielfalt funktioneller Merkmale in Artgemeinschaften. Ich stellte die Hypothese auf, dass projizierte Änderungen in der Vielfalt funktioneller Merkmale in Artgemeinschaften der fruchtfressenden Vögel in mittleren Höhen besonders ausgeprägt sein sollten, da sich benachbarte Artgemeinschaften fruchtfressender Vögel in mittleren Höhen stark voneinander unterscheiden. Ich erwartete, dass dies zu einer Verringerung der funktionellen Entsprechung zwischen den zukünftigen 
Artgemeinschaften von Fruchtpflanzen und fruchtfressenden Vögeln führen würde. Meine Projektionen bestätigten diese Hypothese. Wenn ich in den Simulationen annahm, dass die Fruchtpflanzen und die fruchtfressenden Vogelarten empfindlich gegenüber dem Klimawandel aber limitiert in ihrer Ausbreitungsfähigkeit in höhere Lagen wären, nahm die funktionelle Entsprechung zwischen Artgemeinschaften der Fruchtpflanzen und fruchtfressenden Vögel am stärksten ab. Die niedrige funktionelle Entsprechung der Artgemeinschaften deutet an, dass Pflanzenarten mit bestimmten funktionellen Merkmalen in zukünftigen Artgemeinschaften keine kompatiblen fruchtfressenden Vogelarten finden könnten, was sich negativ auf ihre Samenausbreitung auswirken könnte. Im Gegensatz dazu blieb die funktionelle Entsprechung der Artgemeinschaften hoch, wenn ich in den Simulationen annahm, dass sowohl die Fruchtpflanzenarten als auch die fruchtfressenden Vogelarten nicht empfindlich gegenüber dem Klimawandel wären und gleichzeitig ihre Höhenverbreitung in höhere Lagen ausweiten würden. Meine Ergebnisse zeigen, dass der Erhalt intakter biotischer Wechselwirkungen angesichts des Klimawandels erfordert, dass Arten ihre Verbreitungsgebiete ungehindert in größere Höhen verschieben können.

Im dritten Kapitel meiner Dissertation erkundete ich, ob die Samenausbreitung durch fruchtfressende Vögel ausreicht, damit Fruchtpflanzen geeignete Klimabedingungen zukünftig verfolgen können. Mit merkmalsbasierte Modellen simulierte ich Samenausbreitungsdistanzen, die die fruchtfressenden Vögel für die Fruchtpflanzen bereit stellen könnten. Basierend darauf, berechnete ich die Anzahl an Fernausbreitungsereignissen, die die Pflanzenarten benötigen würden, um den projizierte Temperaturverschiebungen entlang der Höhe vollständig zu folgen. Ich nahm an, dass Fruchtpflanzen in Samenausbreitungssystemen mit einem niedrigeren Grad an Übereinstimmung funktioneller Merkmale, Temperaturverschiebungen besser verfolgen könnten als Fruchtpflanzen in Systemen mit einem hohen Grad an Übereinstimmung funktioneller Merkmale. Des Weiteren erwartete ich, dass großfruchtige Pflanzenarten Verschiebungen in ihrem geeigneten Klima besser verfolgen könnten als kleinfruchtige Arten, da großfruchtige Pflanzenarten bevorzugt von großen Vogelarten verbreitet werden, die die Samen über weite Strecken transportieren. Meine Simulationen zeigten, dass die meisten Fruchtpflanzenarten zahlreiche Fernausbreitungsereignisse benötigen, um den projizierten Verschiebungen in den Klimabedingungen vollständig zu folgen. Dies deutet 
daraufhin, dass die Samenausbreitung durch Vögel unzureichend sein könnte, um es den Pflanzen zu ermöglichen, schnell genug auf den Klimawandel zu reagieren. Zwischen zwei Samenausbreitungsereignissen gibt es eine Zeitverzögerung, in der die Pflanze sich etablieren, heranwachsen und Früchte produzieren muss, bevor sie erneut ausgebreitet werden kann. Dieser Zeitraum kann sich besonders bei langlebigen, holzigen Pflanzenarten über mehrere Jahrzehnte erstrecken. Dies macht es unwahrscheinlich, dass solche Pflanzenarten mehrere aufeinanderfolgende Ausbreitungsereignisse innerhalb einer vergleichsweise kurzen Zeit durchlaufen können. Wie erwartet zeigten meine Simulationen auch, dass Pflanzenarten in Samenausbreitungssystemen mit einem niedrigeren Grad an Übereinstimmung funktioneller Merkmale weniger Fernverbreitungsereignisse zur Verfolgung der projizierten Temperaturverschiebungen benötigten als Pflanzenarten in Systemen mit einem hohen Grad an Übereinstimmung funktioneller Merkmale. Gleichermaßen brauchten großfruchtige Pflanzen weniger Fernausbreitungsereignisse zur Verfolgung der projizierten Temperaturverschiebungen als kleinfruchtige Arten. Insgesamt deutet dies darauf hin, dass die Fähigkeit vogelverbreiteter Pflanzenarten den raschen Klimawandel zu verfolgen, von der Spezialisierung der Samenausbreitungssysteme und den funktionellen Merkmale der Pflanzenarten abhängen.

Die merkmalsbasierten Konzepte und Analysen, die ich in dieser Dissertation entwickelt habe, können sehr generell eingesetzt werden, z. B. um den Einfluss des Klimawandels auf andere biotische Wechselbeziehungen oder den Einfluss anderer Treiber des globalen Wandels zu untersuchen. Nicht zuletzt können merkmalsbasierte Konzepte auch weiterentwickelt werden, um Veränderungen biotischer Wechselbeziehungen dynamisch zu modellieren. Die Weiterentwicklung dieser Konzepte und der daraus resultierende Erkenntnisgewinn sind notwendig, um Projektionen biologischer Vielfalt zu verbessern und Naturschutzmaßnahmen zu informieren. 


\section{References}

Abrams, P.A. (2000). The evolution of predator-prey interactions: theory and evidence. Annu. Rev. Ecol. Syst., 31, 79-105.

Aizen, M.A., Sabatino, M. \& Tylianakis, J.M. (2012). Specialization and rarity predict nonrandom loss of interactions from mutualist networks. Science, 335, 14861489.

Alexander, J.M., Chalmandrier, L., Lenoir, J., Burgess, T.I., Essl, F., Haider, S., et al. (2018). Lags in the response of mountain plant communities to climate change. Glob. Chang. Biol., 24, 563-579.

Algar, A.C. \& Tarr, S. (2018). Fossils, phylogenies and the evolving climate niche. Nat. Ecol. Evol., 2, 414-415.

Araújo, M.B. \& Guisan, A. (2006). Five (or so) challenges for species distribution modelling. J. Biogeogr., 33, 1677-1688.

Ash, J.D., Givnish, T.J. \& Waller, D.M. (2017). Tracking lags in historical plant species' shifts in relation to regional climate change. Glob. Chang. Biol., 23, 1305-1315.

Bagchi, R., Crosby, M., Huntley, B., Hole, D.G., Butchart, S.H.M., Collingham, Y., et al. (2013). Evaluating the effectiveness of conservation site networks under climate change: accounting for uncertainty. Glob. Chang. Biol., 19, 1236-1248.

Barnagaud, J.-Y., Devictor, V., Jiguet, F., Barbet-Massin, M., Le Viol, I. \& Archaux, F. (2012). Relating habitat and climatic niches in birds. PLoS One, 7, e32819.

Barnagaud, J.Y., Kissling, W.D., Tsirogiannis, C., Fisikopoulos, V., Villéger, S., Sekercioglu, C.H., et al. (2017). Biogeographical, environmental and anthropogenic determinants of global patterns in bird taxonomic and trait turnover. Glob. Ecol. Biogeogr., 26, 1190-1200.

Bartomeus, I., Gravel, D., Tylianakis, J.M., Aizen, M.A., Dickie, I.A. \& Bernard-Verdier, M. (2016). A common framework for identifying linkage rules across different types of interactions. Funct. Ecol., 30, 1894-1903.

Bascompte, J. \& Jordano, P. (2007). Plant-animal mutualistic networks: the architecture of biodiversity. Annu. Rev. Ecol. Evol. Syst., 38, 567-593.

Bawa, K.S. (1990). Plant-pollinator interactions in tropical rain forests. Annu. Rev. Ecol. Syst., 21, 399-422.

Bender, I.M.A., Kissling, W.D., Blendinger, P.G., Böhning-Gaese, K., Hensen, I., Kühn, I., et al. (2018). Morphological trait matching shapes plant-frugivore networks across the Andes. Ecography, 41, 1-10. 
Bender, I.M.A., Kissling, W.D., Böhning-Gaese, K., Hensen, I., Kühn, I., Nowak, L., et al. (2019). Projected impacts of climate change on functional diversity of frugivorous birds along a tropical elevational gradient. Sci. Rep., 9, 17708.

Bender, I.M.A., Kissling, W.D., Böhning-Gaese, K., Hensen, I., Kühn, I., Wiegand, T., et al. (2017). Functionally specialised birds respond flexibly to seasonal changes in fruit availability. J. Anim. Ecol., 86, 800-811.

Blonder, B., Lamanna, C., Violle, C. \& Enquist, B.J. (2014). The n-dimensional hypervolume. Glob. Ecol. Biogeogr., 23, 595-609.

Bogoni, J.A., Peres, C.A. \& Ferraz, K.M.P.M.B. (2020). Extent, intensity and drivers of mammal defaunation: a continental-scale analysis across the Neotropics. Sci. Rep., 10, 14750.

Bowler, D., Hof, C., Haase, P., Kröncke, I., Schweiger, O., Adrian, R., et al. (2017). Cross-realm assessment of climate change impacts on species' abundance trends. Nat. Ecol. Evol., 1, 1-7.

Brako, L. \& Zarucchi, J.L. (1993). Catalogue of the flowering plants and Gymnosperms of Peru. Catálogo de las Angiospermas y Gimnospermas del Perú. Monogr. Syst. Bot. from Missouri Bot. Gard. 45.

Brodie, J.F., Aslan, C.E., Rogers, H.S., Redford, K.H., Maron, J.L., Bronstein, J.L., et al. (2014). Secondary extinctions of biodiversity. Trends Ecol. Evol., 29, 664-672.

Brousseau, P.M., Gravel, D. \& Handa, I.T. (2018). Trait matching and phylogeny as predictors of predator-prey interactions involving ground beetles. Funct. Ecol., 32, 192-202.

Burns, K.C. (2013). What causes size coupling in fruit-frugivore interaction webs? Ecology, 94, 295-300.

Cain, M.L., Milligan, B.G. \& Strand, A.E. (2000). Long-distance seed dispersal in plant populations. Am. J. Bot., 87, 1217-1227.

Carlo, T.A. \& Morales, J.M. (2016). Generalist birds promote tropical forest regeneration and increase plant diversity via rare-biased seed dispersal. Ecology, 97, 1819-1831.

Chen, I.C., Hill, J.K., Ohlemüller, R., Roy, D.B. \& Thomas, C.D. (2011). Rapid range shifts of species associated with high levels of climate warming. Science, 333, 1024-1026.

Chen, S., Tamme, R., Thomson, F.J. \& Moles, A.T. (2019). Seeds tend to disperse further in the tropics. Ecol. Lett., 22, 954-961. 
Colwell, R.K., Brehm, G., Cardelús, C.L., Gilman, A.C. \& Longino, J.T. (2008). Global warming, elevational range shifts, and lowland biotic attrition in the wet tropics. Science, 322, 258-261.

Colwell, R.K., Dunn, R.R. \& Harris, N.C. (2012). Coextinction and persistence of dependent species in a changing world. Annu. Rev. Ecol. Evol. Syst., 43, 183203.

Corlett, R.T. (2011). Impacts of warming on tropical lowland rainforests. Trends Ecol. Evol., 26, 606-613.

Corlett, R.T. \& Westcott, D.A. (2013). Will plant movements keep up with climate change? Trends Ecol. Evol., 28, 482-488.

Correa, S.B., Arujo, J.K., Penha, J., Da Cunha, C.N., Bobier, K.E. \& Anderson, J.T. (2016). Stability and generalization in seed dispersal networks: a case study of frugivorous fish in Neotropical wetlands. Proc. R. Soc. B Biol. Sci., 283, 20161267.

Culp, L.A., Cohen, E.B., Scarpignato, A.L., Thogmartin, W.E. \& Marra, P.P. (2017). Full annual cycle: climate change vulnerability assessment for migratory birds. Ecosphere, 8, e01565.

Dalsgaard, B., Schleuning, M., Maruyama, P.K., Dehling, D.M., Sonne, J., VizentinBugoni, J., et al. (2017). Opposed latitudinal patterns of network-derived and dietary specialization in avian plant-frugivore interaction systems. Ecography, 40, 1395-1401.

Dawideit, B.A., Phillimore, A.B., Laube, I., Leisler, B. \& Böhning-Gaese, K. (2009). Ecomorphological predictors of natal dispersal distances in birds. J. Anim. Ecol., 78, 388-395.

Dawson, T.P., House, J.I., Prentice, I.C., Jackson, S.T., Mace, G.M. \& Dawson, T.P. (2011). Beyond predictions: biodiversity conservation in a changing climate. Science, 332, 53-58.

Dehling, D.M., Fritz, S.A., Töpfer, T., Päckert, M., Estler, P., Böhning-Gaese, K., et al. (2014a). Functional and phylogenetic diversity and assemblage structure of frugivorous birds along an elevational gradient in the tropical Andes. Ecography, 37, 1047-1055.

Dehling, D.M., Sevillano, C.S. \& Morales, L.V. (2013). Upper and lower elevational extremes of Andean birds from south-east Peru. Boletín Inf., 8, 32-38.

Dehling, D.M., Töpfer, T., Schaefer, H.M., Jordano, P., Böhning-Gaese, K. \& Schleuning, M. (2014b). Functional relationships beyond species richness 
patterns: trait matching in plant-bird mutualisms across scales. Glob. Ecol. Biogeogr., 23, 1085-1093.

Díaz, S., Lavorel, S., de Bello, F., Quétier, F., Grigulis, K. \& Robson, T.M. (2007). Incorporating plant functional diversity effects in ecosystem service assessments. Proc. Natl. Acad. Sci., 104, 20684-20689.

Díaz, S., Settele, J., Brondízio, E.S., Ngo, H.T., Agard, J., Arneth, A., et al. (2019). Pervasive human-driven decline of life on Earth points to the need for transformative change. Science, 366, eaax3100.

Dirzo, R., Young, H.S., Galetti, M., Ceballos, G., Isaac, N.J.B. \& Collen, B. (2014). Defaunation in the Anthropocene. Science, 345, 401-406.

Donoso, I., Schleuning, M., García, D. \& Fründ, J. (2017). Defaunation effects on plant recruitment depend on size matching and size trade-offs in seed-dispersal networks. Proc. R. Soc. B Biol. Sci., 284, 20162664.

Donoso, I., Sorensen, M.C., Blendinger, P.G., Kissling, W.D., Neuschulz, E.L., Mueller, T., et al. (2020). Downsizing of animal communities triggers stronger functional than structural decay in seed-dispersal networks. Nat. Commun., 11, 1-8.

Dugger, P.J., Blendinger, P.G., Böhning-Gaese, K., Chama, L., Correia, M., Dehling, D.M., et al. (2019). Seed-dispersal networks are more specialized in the Neotropics than in the Afrotropics. Glob. Ecol. Biogeogr., 28, 248-261.

Dunning, J.B. (2007). CRC Handbook of Avian Body Masses. CRC Press, Boca Raton. Eck, S., Töpfer, T., Fiebig, J., Heynen, I., Fiedler, W., Nicolai, B., et al. (2011). Measuring birds. Christ Media Natur, Minden.

Elith, J. \& Leathwick, J.R. (2009). Species distribution models: ecological explanation and prediction across space and time. Annu. Rev. Ecol. Evol. Syst., 40, 677-697. Fang, M., Zhu, X., Du, Y., Zhang, L. \& Lin, L. (2019). Macroecological patterns of climatic niche breadth variation in lacertid lizards. Asian Herpetol. Res., 10, 4147.

Feeley, K.J., Davies, S.J., Perez, R., Hubbell, S.P. \& Foster, R.B. (2011a). Directional changes in the species composition of a tropical forest. Ecology, 92, 871-882.

Feeley, K.J., Silman, M.R., Bush, M.B., Farfan, W., Cabrera, K.G., Malhi, Y., et al. (2011b). Upslope migration of Andean trees. J. Biogeogr., 38, 783-791.

Fleming, T.H. (1979). Do tropical frugivores compete for food? Am. Zool., 19, 11571172.

Foden, W.B., Butchart, S.H.M., Stuart, S.N., Vié, J.C., Akçakaya, H.R., Angulo, A., et 
al. (2013). Identifying the world's most climate change vulnerable species: a systematic trait-based assessment of all birds, amphibians and corals. PLoS One, 8, e65427.

Foden, W.B., Young, B.E., Akçakaya, H.R., Garcia, R.A., Hoffmann, A.A., Stein, B.A., et al. (2018). Climate change vulnerability assessment of species. Wiley Interdiscip. Rev. Clim. Chang., 10, e551.

Forero-Medina, G., Terborgh, J., Socolar, S.J. \& Pimm, S.L. (2011). Elevational ranges of birds on a tropical montane gradient lag behind warming temperatures. PLoS One, 6, e28535.

Freeman, B.G. \& Class Freeman, A.M. (2014). Rapid upslope shifts in New Guinean birds illustrate strong distributional responses of tropical montane species to global warming. Proc. Natl. Acad. Sci., 111, 4490-4494.

Freeman, B.G., Lee-Yaw, J.A., Sunday, J.M. \& Hargreaves, A.L. (2018a). Expanding, shifting and shrinking: the impact of global warming on species' elevational distributions. Glob. Ecol. Biogeogr., 27, 1268-1276.

Freeman, B.G., Scholer, M.N., Ruiz-Gutierrez, V. \& Fitzpatrick, J.W. (2018b). Climate change causes upslope shifts and mountaintop extirpations in a tropical bird community. Proc. Natl. Acad. Sci., 115, 11982-11987.

Fründ, J., McCann, K.S. \& Williams, N.M. (2016). Sampling bias is a challenge for quantifying specialization and network structure: lessons from a quantitative niche model. Oikos, 125, 502-513.

Gagic, V., Bartomeus, I., Jonsson, T., Taylor, A., Winqvist, C., Fischer, C., et al. (2015). Functional identity and diversity of animals predict ecosystem functioning better than species-based indices. Proc. R. Soc. B Biol. Sci., 282, 20142620.

Galetti, M., Guevara, R., Côrtes, M.C., Fadini, R., Matter, S. Von, Leite, A.B., et al. (2013). Functional extinction of birds drives rapid evolutionary changes in seed size. Science, 340, 1086-1090.

Galetti, M., Pires, A.S., Brancalion, P.H.S. \& Fernandez, F.A.S. (2017). Reversing defaunation by trophic rewilding in empty forests. Biotropica, 49, 5-8.

Gallagher, A.J., Hammerschlag, N., Cooke, S.J., Costa, D.P. \& Irschick, D.J. (2015). Evolutionary theory as a tool for predicting extinction risk. Trends Ecol. Evol., 30, 61-65.

Garibaldi, L.A., Bartomeus, I., Bommarco, R., Klein, A.M., Cunningham, S.A., Aizen, M.A., et al. (2015). Trait matching of flower visitors and crops predicts fruit set 
better than trait diversity. J. Appl. Ecol., 52, 1436-1444.

GBIF.org. (2017). GBIF occurence download. Available at: https://www.gbif.org/. Last accessed 20 July 2001.

Ghalambor, C.K., Huey, R.B., Martin, P.R., Tewksbury, J.J. \& Wang, G. (2006). Are mountain passes higher in the tropics? janzen's hypothesis revisited. Integr. Comp. Biol., 46, 5-17.

Gillingham, P.K., Bradbury, R.B., Roy, D.B., Anderson, B.J., Baxter, J.M., Bourn, N.A.D., et al. (2015). The effectiveness of protected areas in the conservation of species with changing geographical ranges. Biol. J. Linn. Soc., 115, 707-717.

Girardin, C.A.J., Aragão, L.E.O.C., Malhi, Y., Huasco, W.H., Metcalfe, D.B., Durand, L., et al. (2013). Fine root dynamics along an elevational gradient in tropical Amazonian and Andean forests. Global Biogeochem. Cycles, 27, 252-264.

Girardin, C.A.J., Malhi, Y., Aragao, L., Mamani, M., Huaraca Huasco, W. \& Durand, L. (2010). Net primary productivity allocation and cycling of carbon along a tropical forest elevational transect in the Peruvian Andes. Glob. Chang. Biol., 16, 31763192.

Githiru, M., Lens, L., Bennur, L.A. \& Ogol, C.P.K.O. (2002). Effects of site and fruit size on the composition of avian frugivore assemblages in a fragmented Afrotropical forest. Oikos, 96, 320-330.

González-Castro, A., Traveset, A. \& Nogales, M. (2012). Seed dispersal interactions in the Mediterranean Region: contrasting patterns between islands and mainland. J. Biogeogr., 39, 1938-1947.

González-Varo, J.P., López-Bao, J. V \& Guitián, J. (2017). Seed dispersers help plants to escape global warming. Oikos, 126, 1600-1606.

Gravel, D., Poisot, T., Albouy, C., Velez, L. \& Mouillot, D. (2013). Inferring food web structure from predator-prey body size relationships. Methods Ecol. Evol., 4, 1083-1090.

Guisan, A. \& Thuiller, W. (2005). Predicting species distribution: offering more than simple habitat models. Ecol. Lett., 8, 993-1009.

Guo, F., Lenoir, J. \& Bonebrake, T.C. (2018). Land-use change interacts with climate to determine elevational species redistribution. Nat. Commun., 9, 1315.

Hannah, L., Roehrdanz, P.R., Marquet, P.A., Enquist, B.J., Midgley, G., Foden, W., et al. (2020). 30\% land conservation and climate action reduces tropical extinction risk by more than 50\%. Ecography, 43, 943-953. 
Hanz, D.M., Böhning-Gaese, K., Ferger, S.W., Fritz, S.A., Neuschulz, E.L., Quitián, M., et al. (2019). Functional and phylogenetic diversity of bird assemblages are filtered by different biotic factors on tropical mountains. J. Biogeogr., 46, 291-303. Heikkinen, R.K., Luoto, M., Leikola, N., Pöyry, J., Settele, J., Kudrna, O., et al. (2010). Assessing the vulnerability of European butterflies to climate change using multiple criteria. Biodivers. Conserv., 19, 695-723.

Heinen, J.H., Rahbek, C. \& Borregaard, M.K. (2020). Conservation of species interactions to achieve self-sustaining ecosystems. Ecography, 43, 1603-1611.

Herrera, C.M. (2002). Seed dispersal by vertebrates. Plant-animal Interact. an Evol. approach. Blackwell Science.

Hijmans, R.J., Cameron, S.E., Parra, J.L., Jones, P.G. \& Jarvis, A. (2005). Very high resolution interpolated climate surfaces for global land areas. Int. J. Climatol., 25, 1965-1978.

Hof, C., Voskamp, A., Biber, M.F., Böhning-Gaese, K., Engelhardt, E.K., Niamir, A., et al. (2018). Bioenergy cropland expansion may offset positive effects of climate change mitigation for global vertebrate diversity. Proc. Natl. Acad. Sci., 115, 13294-13299.

Hooper, D.U., Chapin, III, F.S., Ewel, J.J., Hector, A., Inchausti, P., Lavorel, S., et al. (2005). Effects of biodiversity on ecosystem functioning: a consensus of current knowledge. Ecol. Monogr., 75, 3-35.

Howe, H.F. \& Smallwood, J. (1982). Ecology of seed dispersal. Annu. Rev. Ecol. Syst., 13, 201-228.

IPCC. (2007). Climate Change 2007: synthesis report. Contribution of working groups I, II and III to the fourth assessment report of the Intergovernmental Panel on Climate Change [Core Writing Team, Pachauri, R.K and Reisinger, A. (eds.)]. Geneva, Switzerland.

IPCC. (2014). Climate change 2014: synthesis report. Contribution of working groups I, II and III to the fifth assessment report of the Intergovernmental Panel on Climate Change [Core Writing Team, R.K. Pachauri and L.A. Meyer (eds.)]. Geneva, Switzerland.

IPCC. (2018). Global Warming of $1.5^{\circ} \mathrm{C}$.An IPCC Special Report on the impacts of global warming of $1.5^{\circ} \mathrm{C}$ above pre-industrial levels and related global greenhouse gas emission pathways, in the context of strengthening the global response to the threat of climate change, . 
Jamieson, M.A., Burkle, L.A., Manson, J.S., Runyon, J.B., Trowbridge, A.M. \& Zientek, J. (2017). Global change effects on plant-insect interactions: the role of phytochemistry. Curr. Opin. Insect Sci., 23, 70-80.

Jankowski, J.E., Londoño, G.A., Robinson, S.K. \& Chappell, M.A. (2013a). Exploring the role of physiology and biotic interactions in determining elevational ranges of tropical animals. Ecography, 36, 001-002.

Jankowski, J.E., Merkord, C.L., Rios, W.F., Cabrera, K.G., Revilla, N.S. \& Silman, M.R. (2013b). The relationship of tropical bird communities to tree species composition and vegetation structure along an Andean elevational gradient. J. Biogeogr., 40, 950-962.

Jankowski, J.E., Robison, S.K. \& Levey, D.J. (2010). Squeezed at the top: interspecific aggression may constrain elevational ranges in tropical birds. Ecology, 91, 18771884.

Jansson, R. \& Davies, T.J. (2008). Global variation in diversification rates of flowering plants : energy vs. climate change. Ecol. Lett., 11, 173-183.

Janzen, D.H. (1967). Why mountain passes are higher in the tropics. Am. Nat., 101, 233-249.

Jordano, P. (2014). Fruits and frugivory. In: Seeds: the ecology of regeneration in plant communities. pp. 18-61.

Jordano, P., Forget, P.M., Lambert, J.E., Böhning-Gaese, K., Traveset, A. \& Wright, S.J. (2011). Frugivores and seed dispersal: mechanisms and consequences for biodiversity of a key ecological interaction. Biol. Lett., 7, 321-323.

Jordano, P., Garcia, C., Godoy, J.A. \& Garcia-Castano, J.L. (2007). Differential contribution of frugivores to complex seed dispersal patterns. Proc. Natl. Acad. Sci., 104, 3278-3282.

Karger, D.N., Conrad, O., Böhner, J., Kawohl, T., Kreft, H., Soria-Auza, R.W., et al. (2017). Climatologies at high resolution for the earth's land surface areas. Sci. Data, 4, 1-20.

Kattge, J. \& et al. (2020). TRY plant trait database - enhanced coverage and open access. Glob. Chang. Biol., 119-188.

Khaliq, I., Hof, C., Prinzinger, R., Böhning-Gaese, K. \& Pfenninger, M. (2014). Global variation in thermal tolerances and vulnerability of endotherms to climate change. Proc. R. Soc. B Biol. Sci., 281, 20141097.

Kharouba, H.M., Johan, E., Gelman, A., Bolmgren, K., Allen, J.M., Travers, S.E., et al. 
(2018). Global shifts in the phenological synchrony of species interactions over recent decades. Proc. Natl. Acad. Sci., 115, 5211-5216.

Kissling, D.W., Böhning-Gaese, K. \& Jetz, W. (2009). The global distribution of frugivory in birds. Glob. Ecol. Biogeogr., 18, 150-162.

Kissling, W.D., Walls, R., Bowser, A., Jones, M.O., Kattge, J., Agosti, D., et al. (2018).

Towards global data products of Essential Biodiversity Variables on species traits. Nat. Ecol. Evol., 2, 1531-1540.

Knight, C.A., Blois, J.L., Blonder, B., Macias-Fauria, M., Ordonez, A. \& Svenning, J.C. (2020). Community assembly and climate mismatch in Late Quaternary eastern North American pollen assemblages. Am. Nat., 195, 166-180.

Körner, C. (2000). Why are there global gradients in species richness? Mountains might hold the answer. Trends Ecol. Evol., 15, 513-514.

Lambin, E.F., Geist, H.J. \& Lepers, E. (2003). Dynamics of land-use and land-cover change in tropical regions. Annu. Rev. Environ. Resour., 28, 205-241.

Lenoir, J., Bertrand, R., Comte, L., Bourgeaud, L., Hattab, T., Murienne, J., et al. (2020). Species better track climate warming in the oceans than on land. Nat. Ecol. Evol., 4, 1044-1059.

Lenoir, J. \& Svenning, J.C. (2015). Climate-related range shifts - a global multidimensional synthesis and new research directions. Ecography, 38, 15-28.

Lindström, Å., Green, M., Paulson, G., Smith, H.G. \& Devictor, V. (2013). Rapid changes in bird community composition at multiple temporal and spatial scales in response to recent climate change. Ecography, 36, 313-322.

Londoño, G.A., Chappell, M.A., Jankowski, J.E. \& Robinson, S.K. (2017). Do thermoregulatory costs limit altitude distributions of Andean forest birds? Funct. Ecol., 31, 204-215.

Luck, G.W., Lavorel, S., McIntyre, S. \& Lumb, K. (2012). Improving the application of vertebrate trait-based frameworks to the study of ecosystem services. J. Anim. Ecol., 81, 1065-1076.

Lustenhouwer, N., Moran, E. V \& Levine, J.M. (2017). Trait correlations equalize spread velocity across plant life histories. Glob. Ecol. Biogeogr., 26, 1398-1407.

MacLean, S.A. \& Beissinger, S.R. (2017). Species' traits as predictors of range shifts under contemporary climate change: a review and meta-analysis. Glob. Chang. Biol., 23, 4094-4105.

Maglianesi, M.A., Blüthgen, N., Böhning-Gaese, K. \& Schleuning, M. (2014). 
Morphological traits determine specialization and resource use in planthummingbird networks in the neotropics. Ecology, 95, 3325-3334.

Mahony, C.R., Cannon, A.J., Wang, T. \& Aitken, S.N. (2017). A closer look at novel climates: new methods and insights at continental to landscape scales. Glob. Chang. Biol., 23, 3934-3955.

Mamantov, M.A., Gibson-Reinemer, D.K., Linck, E.B. \& Sheldon, K.S. (2021). Climatedriven range shifts of montane species vary with elevation. Glob. Ecol. Biogeogr., $00,1-11$.

Markl, J.S., Schleuning, M., Forget, P.M., Jordano, P., Lambert, J.E., Traveset, A., et al. (2012). Meta-analysis of the effects of human disturbance on seed dispersal by animals. Conserv. Biol., 26, 1072-1081.

Menke, S., Böhning-Gaese, K. \& Schleuning, M. (2012). Plant-frugivore networks are less specialized and more robust at forest-farmland edges than in the interior of a tropical forest. Oikos, 121, 1553-1566.

Merkord, C.L. (2010). Seasonality and elevational migration in an Andean bird community. University of Missouri-Columbia.

Miele, V. \& Matias, C. (2017). Revealing the hidden structure of dynamic ecological networks. R. Soc. open Sci., 4, 170251.

Moermond, T.C. \& Denslow, J.S. (1985). Neotropical avian frugivores: patterns of behavior, morphology, and nutrition, with consequences for fruit selection. Ornithol. Monogr., 36, 865-897.

Mokany, K., Prasad, S. \& Westcott, D.A. (2015). Impacts of climate change and management responses in tropical forests depend on complex frugivore-mediated seed dispersal. Glob. Ecol. Biogeogr., 24, 685-694.

Mokhov, I.I. \& Akperov, M.G. (2006). Tropospheric lapse rate and its relation to surface temperature from reanalysis data. Izv. Atmos. Ocean. Phys., 42, 430-438.

Moritz, C., Patton, J.L., Conroy, C.J., Parra, J.L., White, G.C. \& Beissinger, S.R. (2008). Impact of a century of climate change on small-mammal communities in Yosemite National Park, USA. Science, 322, 261-264.

Morueta-Holme, N., Engemann, K., Sandoval-Acuña, P., Jonas, J.D., Segnitz, R.M. \& Svenning, J.-C. (2015). Strong upslope shifts in Chimborazo's vegetation over two centuries since Humboldt. Proc. Natl. Acad. Sci., 112, 12741-12745.

Di Musciano, M., Di Cecco, V., Bartolucci, F., Conti, F., Frattaroli, A.R. \& Di Martino, L. (2020). Dispersal ability of threatened species affects future distributions. Plant 
Ecol., 221, 265-281.

Myers, N., Mittermeier, R.A., Mittermeier, C.G., da Fonseca, G.A.B. \& Kent, J. (2000). Biodiversity hotspots for conservation priorities. Nature, 403, 853-858.

Naoe, S., Tayasu, I., Sakai, Y., Masaki, T., Kobayashi, K., Nakajima, A., et al. (2016). Mountain-climbing bears protect cherry species from global warming through vertical seed dispersal. Curr. Biol., 26, R315-R316.

Nathan, R., Schurr, F.M., Spiegel, O., Steinitz, O., Trakhtenbrot, A. \& Tsoar, A. (2008). Mechanisms of long-distance seed dispersal. Trends Ecol. Evol., 23, 638-647.

Newbold, T. (2018). Future effects of climate and land-use change on terrestrial vertebrate community diversity under different scenarios. Proc. R. Soc. B Biol. Sci., 285, 20180792.

Norden, N., Chazdon, R.L., Chao, A., Jiang, Y.H. \& Vílchez-Alvarado, B. (2009). Resilience of tropical rain forests: tree community reassembly in secondary forests. Ecol. Lett., 12, 385-394.

Nowak, L., Kissling, W.D., Bender, I.M.A., Dehling, D.M., Töpfer, T., Böhning-Gaese, $\mathrm{K}$., et al. (2019). Projecting consequences of global warming for the functional diversity of fleshy-fruited plants and frugivorous birds along a tropical elevational gradient. Divers. Distrib., 25, 1362-1374.

Nunez, S., Arets, E., Alkemade, R., Verwer, C. \& Leemans, R. (2019). Assessing the impacts of climate change on biodiversity: is below $2{ }^{\circ} \mathrm{C}$ enough? Clim. Change, 154, 351-365.

Onstein, R.E., Baker, W.J., Couvreur, T.L.P., Faurby, S., Herrera-Alsina, L., Svenning, J.-C., et al. (2018). To adapt or go extinct? The fate of megafaunal palm fruits under past global change. Proc. R. Soc. B Biol. Sci., 285, 20180882.

Pacifici, M., Foden, W.B., Visconti, P., Watson, J.E.M., Butchart, S.H.M., Kovacs, K.M., et al. (2015). Assessing species vulnerability to climate change. Nat. Clim. Chang., $5,215-224$.

Parmesan, C. \& Yohe, G. (2003). A globally coherent fingerprint of climate change impacts across natural systems. Nature, 421, 37-42.

Patterson, B.D., Stotz, D.F., Solarit, S., Fitzpatrick, J.W. \& Pacheco, V. (1998). Contrasting patterns of elevational zonation for birds and mammals in the Andes of southeastern Peru. J. Biogeogr., 25, 593-607.

Pecl, G.T., Araújo, M.B., Bell, J.D., Blanchard, J., Bonebrake, T.C., Chen, I.C., et al. (2017). Biodiversity redistribution under climate change: impacts on ecosystems 
and human well-being. Science, 355, eaai9214.

Pichler, M., Boreux, V., Klein, A., Schleuning, M. \& Hartig, F. (2020). Machine learning algorithms to infer trait-matching and predict species interactions in ecological networks. Methods Ecol. Evol., 11, 281-293.

Plein, M., Längsfeld, L., Neuschulz, E.L., Schultheiß, C., Ingmann, L., Töpfer, T., et al. (2013). Constant properties of plant-frugivore networks despite fluctuations in fruit and bird communities in space and time. Ecology, 94, 1296-1306.

Post, E. \& Forchhammer, M.C. (2008). Climate change reduces reproductive success of an Arctic herbivore through trophic mismatch. Philos. Trans. R. Soc. B Biol. Sci., 363, 2369-2375.

Le Provost, G., Gross, N., Börger, L., Deraison, H., Roncoroni, M. \& Badenhausser, I. (2017). Trait-matching and mass effect determine the functional response of herbivore communities to land-use intensification. Funct. Ecol., 31, 1600-1611.

Quintero, E., Pizo, M.A. \& Jordano, P. (2020). Fruit resource provisioning for avian frugivores: The overlooked side of effectiveness in seed dispersal mutualisms. J. Ecol., 108, 1358-1372.

Quitián, M., Santillán, V., Espinosa, C.I., Homeier, J., Böhning-Gaese, K., Schleuning, M., et al. (2018). Elevation-dependent effects of forest fragmentation on plant-bird interaction networks in the tropical Andes. Ecography, 41, 1497-1506.

Rapp, J.M. \& Silman, M.R. (2012). Diurnal, seasonal, and altitudinal trends in microclimate across a tropical montane cloud forest. Clim. Res., 55, 17-32.

Reif, J., Hořák, D., Krištín, A., Kopsová, L. \& Devictor, V. (2016). Linking habitat specialization with species' traits in European birds. Oikos, 125, 405-413.

Renner, S.S. \& Zohner, C.M. (2018). Climate change and phenological mismatch in trophic interactions among plants, insects, and vertebrates. Annu. Rev. Ecol. Evol. Syst., 49, 165-182.

Rubidge, E.M., Patton, J.L., Lim, M., Burton, A.C., Brashares, J.S. \& Moritz, C. (2012). Climate-induced range contraction drives genetic erosion in an alpine mammal. Nat. Clim. Chang., 2, 285-288.

Sales, L.P., Kissling, W.D., Galetti, M., Naimi, B. \& Pires, M. (2021a). Climate change reshapes the eco-evolutionary dynamics of a Neotropical seed dispersal system. Glob. Ecol. Biogeogr., 00, 1-10.

Sales, L.P., Ribeiro, B.R., Pires, M.M., Chapman, C.A. \& Loyola, R. (2019). Recalculating route: dispersal constraints will drive the redistribution of Amazon 
primates in the Anthropocene. Ecography, 42, 1789-1801.

Sales, L.P., Rodrigues, L. \& Masiero, R. (2021b). Climate change drives spatial mismatch and threatens the biotic interactions of the Brazil nut. Glob. Ecol. Biogeogr., 30, 117-127.

Salguero-Gómez, R., Jones, O.R., Archer, C.R., Buckley, Y.M., Che-Castaldo, J., Caswell, H., et al. (2015). The COMPADRE Plant Matrix Database: an open online repository for plant demography. J. Ecol., 103, 202-218.

Salguero-Gómez, R., Jones, O.R., Jongejans, E., Blomberg, S.P., Hodgson, D.J., Mbeau-Ache, C., et al. (2016). Fast-slow continuum and reproductive strategies structure plant life-history variation worldwide. Proc. Natl. Acad. Sci., 113, 230235.

Sanders, N.J. \& Rahbek, C. (2012). The patterns and causes of elevational diversity gradients. Ecography, 35, 1-3.

Santini, L., Butchart, S.H.M., Rondinini, C., Benítez-López, A., Hilbers, J.P., Schipper, A.M., et al. (2019). Applying habitat and population-density models to land-cover time series to inform IUCN Red List assessments. Conserv. Biol., 33, 1084-1093.

Schleuning, M., Fründ, J. \& García, D. (2015). Predicting ecosystem functions from biodiversity and mutualistic networks: An extension of trait-based concepts to plant-animal interactions. Ecography, 38, 380-392.

Schleuning, M., Fründ, J., Klein, A.-M., Abrahamczyk, S., Alarcón, R., Albrecht, M., et al. (2012). Specialization of mutualistic interaction networks decreases toward Tropical latitudes. Curr. Biol., 22, 1925-1931.

Schleuning, M., Fründ, J., Schweiger, O., Welk, E., Albrecht, J., Albrecht, M., et al. (2016). Ecological networks are more sensitive to plant than to animal extinction under climate change. Nat. Commun., 7, 13965.

Schleuning, M., Neuschulz, E.L., Albrecht, J., Bender, I.M.A., Bowler, D.E., Dehling, D.M., et al. (2020). Trait-Based assessments of climate-change impacts on interacting species. Trends Ecol. Evol., 35, 319-328.

Schulenberg, T.S., Stotz, D.F., Lane, D.F., O’Neill, J.P. \& Parker III, T.A. (2010). Birds of Peru: revised and updated edition (Vol. 63). Princeton University Press.

Schweiger, O., Settele, J., Kudrna, O., Klotz, S. \& Kühn, I. (2008). Climate change can cause spatial mismatch of trophically interacting species. Ecology, 89, 34723479 .

Seebens, H., Essl, F., Dawson, W., Fuentes, N., Moser, D., Pergl, J., et al. (2015). 
Global trade will accelerate plant invasions in emerging economies under climate change. Glob. Chang. Biol., 21, 4128-4140.

Shah, A.A., Gill, B.A., Encalada, A.C., Flecker, A.S., Funk, W.C., Guayasamin, J.M., et al. (2017). Climate variability predicts thermal limits of aquatic insects across elevation and latitude. Funct. Ecol., 31, 2118-2127.

Shanahan, M. \& Compton, S.G. (2001). Vertical stratification of figs and fig-eaters in a Bornean lowland rain forest: how is the canopy different? Plant Ecol., 153, 121132.

Sheard, C., Neate-Clegg, M.H.C., Alioravainen, N., Jones, S.E.I., Vincent, C., MacGregor, H.E.A., et al. (2020). Ecological drivers of global gradients in avian dispersal inferred from wing morphology. Nat. Commun., 11, 1-9.

Skellam, J.G. (1951). Random dispersal in theoretical populations. Biometrika, 38, 196-218.

Slatyer, R.A., Hirst, M. \& Sexton, J.P. (2013). Niche breadth predicts geographical range size: a general ecological pattern. Ecol. Lett., 16, 1104-1114.

Sorensen, M.C., Donoso, I., Neuschulz, E.L., Schleuning, M. \& Mueller, T. (2020). Community-wide seed dispersal distances peak at low levels of specialisation in size-structured networks. Oikos, 129, 1727-1738.

La Sorte, F.A. \& Jetz, W. (2010). Projected range contractions of montane biodiversity under global warming. Proc. R. Soc. B Biol. Sci., 277, 3401-3410.

Stam, J.M., Kroes, A., Li, Y., Gols, R., van Loon, J.J.A., Poelman, E.H., et al. (2014). Plant interactions with multiple insect herbivores: from community to genes. Annu. Rev. Plant Biol., 65, 689-713.

Stang, M., Klinkhamer, P.G.L., Waser, N.M., Stang, I. \& Van Der Meijden, E. (2009). Size-specific interaction patterns and size matching in a plant-pollinator interaction web. Ann. Bot., 103, 1459-1469.

Steinbauer, M.J., Grytnes, J.-A., Jurasinski, G., Kulonen, A., Lenoir, J., Pauli, H., et al. (2018). Accelerated increase in plant species richness on mountain summits is linked to warming. Nature, 556, 231-236.

Stevens, G.C. (1989). The latitudinal gradient in geographical range: how so many species coexist in the tropics. Am. Nat., 133, 240-256.

Stevens, G.C. (1992). The elevational gradient in altitudinal range: an extension of Rapoport 's latitudinal rule to altitude. Am. Nat., 140, 893-911.

Stocker, T.F., Qin, D., Plattner, G.-K., Tignor, M.M.B., Allen, S.K., Boschung, J., et al. 
(2014). Climate Change 2013 - The Physical Science Basis. Cambridge University Press, Cambridge.

Strayer, D.L., Eviner, V.T., Jeschke, J.M. \& Pace, M.L. (2006). Understanding the longterm effects of species invasions. Trends Ecol. Evol., 21, 645-651.

Suding, K.N., Lavorel, S., Chapin III, F.S., Cornelissen, J.H.C., Díaz, S., Garnier, E., et al. (2008). Scaling environmental change through the community-level: a traitbased response-and-effect framework for plants. Glob. Chang. Biol., 14, 11251140.

Sunday, J.M., Pecl, G.T., Frusher, S., Hobday, A.J., Hill, N., Holbrook, N.J., et al. (2015). Species traits and climate velocity explain geographic range shifts in an ocean-warming hotspot. Ecol. Lett., 18, 944-953.

Svenning, J.C., Pedersen, P.B.M., Donlan, C.J., Ejrnæs, R., Faurby, S., Galetti, M., et al. (2016). Science for a wilder Anthropocene: Synthesis and future directions for trophic rewilding research. Proc. Natl. Acad. Sci. U. S. A., 113, 898-906.

Tewksbury, J.J., Levey, D.J., Haddad, N.M., Sargent, S., Orrock, J.L., Weldon, A., et al. (2002). Corridors affect plants, animals, and their interactions in fragmented landscapes. Proc. Natl. Acad. Sci. U. S. A., 99, 12923-12926.

Thomson, F.J., Moles, A.T., Auld, T.D. \& Kingsford, R.T. (2011). Seed dispersal distance is more strongly correlated with plant height than with seed mass. J. Ecol., 99, 1299-1307.

Thuiller, W., Lavergne, S., Roquet, C., Boulangeat, I., Lafourcade, B. \& Araujo, M.B. (2011). Consequences of climate change on the tree of life in Europe. Nature, 470, 531-534.

Thuiller, W., Lavorel, S., Araujo, M.B., Sykes, M.T. \& Prentice, I.C. (2005). Climate change threats to plant diversity in Europe. Proc. Natl. Acad. Sci., 102, 82458250 .

Thurman, L.L., Stein, B.A., Beever, E.A., Foden, W., Geange, S.R., Green, N., et al. (2020). Persist in place or shift in space? Evaluating the adaptive capacity of species to climate change. Front. Ecol. Environ., 18, 520-528.

Tilman, D. (2001). Functional Diversity. Encycl. Biodivers., 3, 109-120.

Trisos, C.H., Merow, C. \& Pigot, A.L. (2020). The projected timing of abrupt ecological disruption from climate change. Nature, 580, 496-501.

Tropicos. (2017). Tropicos. Missouri Bot. Gard. Available at: http://www.tropicos.org. Last accessed 15 March 2017. 
Tylianakis, J.M., Laliberté, E., Nielsen, A. \& Bascompte, J. (2010). Conservation of species interaction networks. Biol. Conserv., 143, 2270-2279.

Urban, M.C. (2015). Accelerating extinction risk from climate change. Science, 348, 571-573.

Urban, M.C., Bocedi, G., Hendry, A.P., Mihoub, J.B., Peer, G., Singer, A., et al. (2016). Improving the forecast for biodiversity under climate change. Science, 353, aad8466-aad8466.

Villéger, S., Mason, N.W.H. \& Mouillot, D. (2008). New multidimensional functional diversity indices for a multifaceted framework in functional ecology. Ecology, 89, 2290-2301.

Violle, C., Navas, M.-L., Vile, D., Kazakou, E., Fortunel, C., Hummel, I., et al. (2007). Let the concept of trait be functional! Oikos, 116, 882-892.

Vizentin-Bugoni, J., Debastiani, V.J., Bastazini, V.A.G., Maruyama, P.K. \& Sperry, J.H. (2020). Including rewiring in the estimation of the robustness of mutualistic networks. Methods Ecol. Evol., 11, 106-116.

Vollstädt, M.G.R., Ferger, S.W., Hemp, A., Howell, K.M., Töpfer, T., Böhning-Gaese, K., et al. (2017). Direct and indirect effects of climate, human disturbance and plant traits on avian functional diversity. Glob. Ecol. Biogeogr., 26, 963-972.

Walker, B., Stotz, D.F., Pequeño, T. \& Fitzpatrick, J.W. (2006). Birds of the Manu Biosphere Reserve. Fieldiana Zool., 23-49.

Wang, B.C. \& Smith, T.B. (2002). Closing the seed dispersal loop. Trends Ecol. Evol., 17, 379-385.

Warren, R., Price, J., Graham, E., Forstenhaeusler, N. \& VanDerWal, J. (2018). The projected effect on insects, vertebrates, and plants of limiting global warming to $1.5^{\circ} \mathrm{C}$ rather than $2^{\circ} \mathrm{C}$. Science, $360,791-795$.

Weinstein, B.G. \& Graham, C.H. (2017). Persistent bill and corolla matching despite shifting temporal resources in tropical hummingbird-plant interactions. Ecol. Lett., 20, 326-335.

Westcott, D.A. \& Graham, D.L. (2000). Patterns of movement and seed dispersal of a tropical frugivore. Oecologia, 122, 249-257.

Wheelwright, N.T. (1985). Fruit-size, gape width, and the diets of fruit-eating birds. Ecology, 66, 808-818.

Winkler, H. \& Leisler, B. (1992). On the ecomorphology of migrants. Ibis (Lond. 1859)., 134, 21-28. 
Wotton, D.M. \& Kelly, D. (2012). Do larger frugivores move seeds further? Body size, seed dispersal distance, and a case study of a large, sedentary pigeon. $J$. Biogeogr., 39, 1973-1983.

Wunderle Jr, J.M. (1997). The role of animal seed dispersal in accelerating native forest regeneration on degraded tropical lands. For. Ecol. Manage., 99, 223-235.

Zarnetske, P.L., Skelly, D.K. \& Urban, M.C. (2012). Biotic multipliers of climate change. Science, 336, 1516-1518. 


\section{Acknowledgements}

I would like to take the opportunity to express my heartfelt gratitude to everyone who supported me during my PhD.

First, I am most grateful to my supervisor Matthias Schleuning. Matthias, thank you so much for your invaluable mentoring throughout these years. Your calm, wellconsidered and structured advice on all types of scientific questions made it a most enjoyable experience to work with you. Thanks to your guidance, I never felt lost on this adventurous path.

Moreover, I would like to thank Daniel Kissling for taking over the role of being my second supervisor and for doing it greatly. Daniel, I am most grateful for your willingness to share your scientific experience. I also want to thank you for welcoming me for a research stay at your lab in Amsterdam. That was a wonderful experience.

I would further like to thank Katrin Böhning-Gaese most warmly for giving me the unique opportunity to conduct my $\mathrm{PhD}$ in her welcoming and well-connected working group at the Senckenberg Biodiversity and Climate Research Centre in Frankfurt am Main.

The Functional Ecology and Global Change lab and the Macroevolution of Birds and Mammals lab both welcomed me most warmly and provided me with enlightening scientific discussions, as well as valuable feedback on my projects. I enjoyed it a lot to be a part of these two great labs.

In addition, my gratitude belongs to Susanne Fritz, Irene Bender and Isabel Donoso. Susanne, it was highly enjoyable and educational to closely collaborate with you for one of my PhD chapters and I am most grateful for your valuable advice on the structure of this dissertation. Moreover, I want to thank you for welcoming me to your Macroevolution of Birds and Mammals lab. Irene, I want to thank you for helping me getting started when doing a $\mathrm{PhD}$ was still new and somewhat overwhelming to me. Your positive attitude towards work and life is absolutely inspiring. Finally, Isa, I am super grateful for your support with my last $\mathrm{PhD}$ chapter. Working with you is a pleasure and your enthusiasm for science is most encouraging.

Special thanks to Matthias Dehling and Irene Bender for allowing me to continue to work with the data you have collected and providing valuable scientific input. Furthermore, I am most grateful to all my co-authors for their ideas, feedback and insightful scientific discussions. 
Matthias Schleuning and Katrin Böhning-Gaese enabled me to visit Peru and the Manú biosphere reserve and I cannot express how grateful I am for that. Experiencing such a diverse and beautiful ecosystem was one of the most impressive adventures in my life. Vicky and Adan, thank you a million for keeping me safe in the rainforest and sharing your knowledge about the fascinating plants and animals dwelling there.

Importantly, I would like to thank Sabine, Leo, Tanja, Matze and Anke for their assistance with all administrative tasks.

Nandia, Alke, Isa and Irene, heartfelt thanks for taking the time to provide me with constructive feedback on my dissertation.

Last but not least, I want to thank my family, friends and partner for their continuing support. Mama, Papa, Bianka, Oskar and little Emily, thank you for being the best, funniest and most supportive family. Kathy, Katha, Henrik, Nandia, Ali and Alke, thank you a bunch for conversations, dinners, coffees, walks, bike tours, bouldering dates, concerts, movie nights, museum visits, and many more fun activities. You made Frankfurt my home and my time here a most enjoyable one. Lou, Hansi and Jana thank you so much for being my friends for many years despite me moving all over the place. Finally, Kai, thank you for your support in graphic design issues, rehearsal of my presentations, study sessions, pep talks, food packages and most importantly for being there. 


\section{Appendices}

A1: Independent variation of avian sensitivity to climate change and adaptive capacity along a tropical elevational gradient

Authors: Larissa Nowak, Matthias Schleuning, Irene. M. A. Bender, W. Daniel Kissling, Susanne A. Fritz

Title: Independent variation of avian sensitivity to climate change and adaptive capacity along a tropical elevational gradient

Status: submitted

Author contributions:

(1) Concept and design

LN, 80\%;

MS, SAF, in total $20 \%$

(2) Conducting tests and experiments

n. a.

(3) Compilation of data sets and figures

IMAB contributed bird elevational distributions and bird morphological data, based on previous studies by Till Töpfer and Matthias Dehling, 100\%;

WDK compiled bird habitat data from literature, 100\%;

IMAB downloaded bird occurrence data, 100\%;

LN, $40 \%$, and IMAB, $60 \%$, processed bird occurrence data;

LN downloaded and processed bioclimatic data, 100\%;

LN prepared all data for analyses, $100 \%$;

LN prepared all figures and tables, $100 \%$

\section{(4) Analysis and interpretation of data}

LN computed species' climatic niche breadths, 100\%;

LN tested for variation of traits along elevation and across species, $100 \%$;

LN interpreted the results, $90 \%$

with input from MS, IMAB, WDK, SAF, in total $10 \%$

\section{(5) Drafting of manuscript}

LN, 85\%;

MS, SAF, in total $10 \%$;

IMAB, WDK, in total $5 \%$ 
Independent variation of avian sensitivity to climate change and adaptive capacity along a tropical elevational gradient

\begin{abstract}
To date, it is largely unknown how species' sensitivity to climate change and their adaptive capacity covary. However, understanding this relationship is crucial to predict the potential consequences of climate change for species assemblages. Here, we test how traits and attributes related to species' sensitivity to climate change and their adaptive capacity (i) vary along a broad elevational gradient and (ii) covary across a large number of bird species. We focus on 215 frugivorous bird species along a Neotropical elevational gradient ranging from 250 to $3750 \mathrm{~m}$ a.s.I. We approximate species' sensitivity to climate change by the climatic niche breadth of species which we estimate with bioclimatic variables and species occurrences across South America. In addition, we focus on ecological aspects of the adaptive capacity of species, especially on the dispersal ability of species (approximated by their wing pointedness), their dietary niche breadth (approximated by bill width), and species' habitat niche breadth (approximated by the number of inhabited habitat classes). We found that (i) species' climatic niche breadth increased with elevation, while their dispersal ability and dietary niche breadth decreased with elevation, and (ii) that species' sensitivity to climate change and their adaptive capacity were not significantly related across species. These results suggest different mechanisms of how species in lowland and highland assemblages might respond to climate change. The independent variation of species' sensitivity to climate change and their adaptive capacity suggests that comprehensive assessments of potential impacts of climate change on species assemblages should consider different dimensions of the susceptibility of species to climate change.
\end{abstract}

\title{
Key words
}

Response traits, birds, climatic niche, dispersal, dietary niche, habitat niche, climate change, functional traits, frugivory, mountain 


\section{Introduction}

Contemporary climate change affects species assemblages globally. Species can respond in different ways to climate change. First, species might persist locally because changing climates lie within their climatic niche or because they adjust to changing climates in situ (Bellard et al. 2012). Second, many species shift their ranges to higher latitudes and elevations to track their suitable climate under climate change (Chen et al. 2011, Lenoir et al. 2020). Moreover, species need to respond to climateinduced changes in resource or habitat availability (Jackson et al. 2015, Maron et al. 2015). Predicting potential impacts of climate change on species assemblages likely requires accounting for the different ways species may respond to the changing conditions.

To understand how species might respond to climate change, we can draw upon a framework that has been described by the Intergovernmental Panel on Climate Change (IPCC 2007) and is commonly applied in assessments of species' vulnerability to climate change (Foden et al. 2013, 2018). According to this framework, the susceptibility of species to climate change comprises three dimensions, i.e., the exposure of species to climate change, their sensitivity and their adaptive capacity. Here, we focus on the two dimensions that relate to intrinsic properties of species and influence their ability to respond to climate change. First, the sensitivity of a species to climate change is defined as the degree to which the species is affected by climate change (IPCC 2007, Foden et al. 2018). This is influenced by the climatic niche of the species, i.e., the climatic conditions in which the species can maintain evolutionary fitness and stable populations (Algar and Tarr 2018). Second, the adaptive capacity of a species is defined as the species' ability to adjust to climate change, respond to its consequences and moderate potential damage (IPCC 2007, 2014, Foden et al. 2018). This is related to several ecological and evolutionary attributes of the species, e.g., its dispersal ability, phenotypic plasticity, and evolutionary potential (Pacifici et al. 2015, Foden et al. 2018).

Studies on potential impacts of climate change on species assemblages often focus on species' sensitivity to climate change, e.g., by assessing occurrence-based or physiologically derived measures of species' climatic niche (Thuiller et al. 2006, Khaliq et al. 2014, Nunez et al. 2019). In contrast, aspects of the adaptive capacity of species have mostly been accounted for in integrated vulnerability measures (Foden et al. 2013, Culp et al. 2017) and are only recently being incorporated into projection 
models (Razgour et al. 2019, Di Musciano et al. 2020). Nevertheless, it remains unclear how the sensitivity of species to climate change relates to their adaptive capacity and consequently whether it is sufficient to study species' responses to climate change exclusively from aspects of sensitivity.

Species' sensitivity and adaptive capacity can be approximated by response traits of the species and related attributes influencing how species respond to environmental change (Luck et al. 2012, Foden et al. 2018). First, the sensitivity of species to climate change can be approximated by measures of species' climatic niche breadth which influences the persistence under climate change (Botts et al. 2013, Foden et al. 2018, Herrera et al. 2018; Fig. 1a). Second, important ecological aspects of the adaptive capacity of species under climate change are their ability to shift their ranges and to utilize different resources. Species' ability for range shifts reflects their ability to track their suitable climate during climate change and can be approximated by measures of species' dispersal ability (Schloss et al. 2012, Sunday et al. 2015). Species' ability to utilize different resources influences whether the species can adjust to changes in resource availability and can be approximated by the ecological niche breadth of species, e.g., their diet and habitat niche breadth (Slatyer et al. 2013; Fig. 1c, d). To understand the potential responses of species to climate change these traits and attributes might need to be assessed in combination.

Knowledge on relationships of species' sensitivity and adaptive capacity at the assemblage level may inform us about the overall ability of species' assemblages to respond to climate change. Previous work suggests that species' sensitivity and adaptive capacity differ among assemblages in different environmental contexts. According to Janzen's climate variability hypothesis, species from variable climates have broader thermal niches than species from stable climates (Janzen 1967, Ghalambor et al. 2006). Accordingly, the thermal tolerance of different animal species tends to increase with increasing latitude and elevation (Khaliq et al. 2014, Shah et al. 2017, Fang et al. 2019). Similarly, geographic patterns of species' dispersal ability and ecological niche breadth have been investigated. For instance, a recent study revealed a positive latitudinal trend of average wing pointedness, a proxy for dispersal ability, in avian assemblages across the globe, possibly driven by increasing climate variability with latitude (Sheard et al. 2020). Furthermore, the latitude-niche breadth hypothesis predicts an increase of species' ecological niche breadth with latitude, but studies that have tested for latitudinal gradients in species' ecological niche breadth report 
inconsistent results (Vázquez and Stevens 2004, Schleuning et al. 2012). Despite the few existing studies of large-scale variation in species' response traits and attributes, relationships between species' sensitivity to climate change and their adaptive capacity have not yet been explicitly tested along environmental gradients.

Furthermore, it is contentious whether species' sensitivity and adaptive capacity are related across species. For instance, the habitat niche breadth of European breeding birds has been reported to be positively related to their dietary niche breadth and negatively related to their climatic niche breadth (Reif et al. 2016). However, habitat niche breadth and climatic niche breadth were positively related in a study focussing on French breeding birds (Barnagaud et al. 2012). Resolving such differences is important because the relationship between species' sensitivity and adaptive capacity determines potential trade-offs in species' ability to respond to climate change. For instance, if species that are highly sensitive to climate change also have a low adaptive capacity, then their overall susceptibility to climate change might be higher than estimated from their climatic niche alone (Foden et al. 2013).

Here, we aim to identify the relationship of species' sensitivity to climate change with species' adaptive capacity and assess this relationship (i) in different assemblages along an elevational gradient and (ii) across species. We focus on 215 avian frugivore species co-occurring along a Neotropical elevational gradient with a highly diverse avifauna. We focus our analyses on avian frugivores because they form a homogeneous ecological guild, hence traits and measures of ecological niches are comparable across species. To approximate species' sensitivity to climate change, we quantify species' climatic niche breadth as hypervolume based on their current occurrences and climate variables across South America (Fig. 1 a). To approximate species' adaptive capacity, we focus on morphological traits related to species' dispersal ability and dietary niche breadth in terms of fruit choice as well as on species' habitat niche breadth (Fig. 1 b-d). We expect that (i) species' climatic niche breadth in frugivore assemblages increases with increasing elevation because increasing diurnal temperature variability favours species with broad climatic niches at high elevations (Ghalambor et al. 2006). Furthermore, we expect that species' dispersal ability and ecological niche breadth decrease with increasing elevation due to environmental filtering of avian frugivore assemblages at high elevations (Dehling et al. 2014a). ii) Across species, we expect a negative relationship of species' sensitivity to climate change and their adaptive capacity, as sensitivity and adaptive capacity might trade 
off in species that have survived past climate change. There is no clear a priori support for this hypothesis.
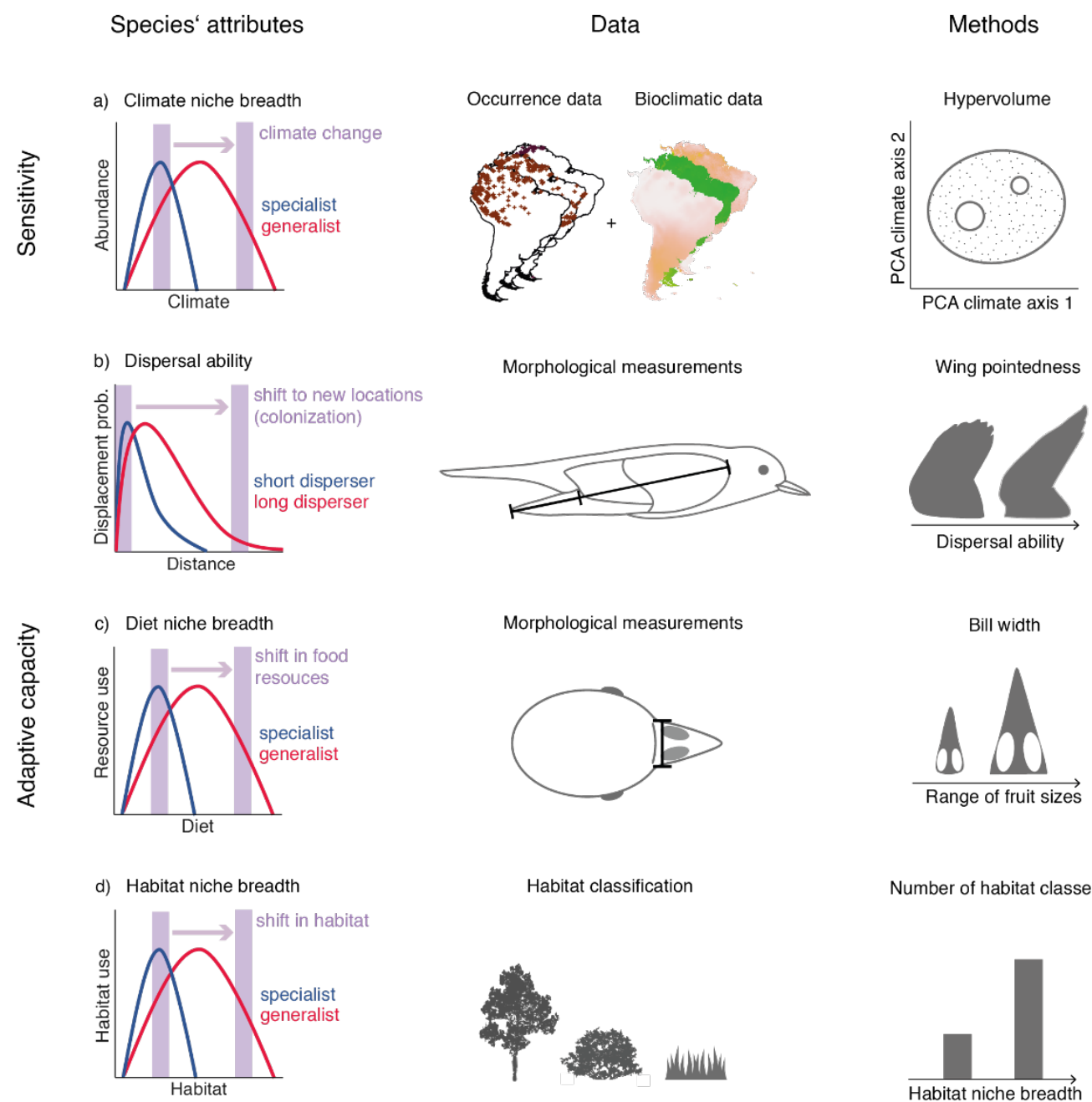

Number of habitat classes

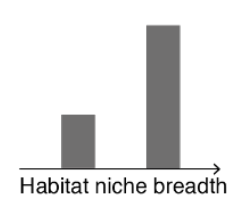

Figure 1: Response traits and attributes related to species' sensitivity to climate change (here climatic niche breadth, a) and their adaptive capacity (here dispersal ability, dietary niche breadth and habitat niche breadth, b-d). (a) A species' sensitivity to climate change can be approximated by its climatic niche breadth since a species with a broad climatic niche (generalist) has a higher chance that changing climates remain within its niche than a species with a narrow climatic niche (specialist). We estimated the climatic niche breadth of the avian frugivores based on species' current occurrences and climate data across South America as a hypervolume in a twodimensional climate space. (b-d) Important aspects of a species' adaptive capacity are the species' ability to shift their ranges and to utilize a wide range of resources. (b) A species' ability to shift their ranges influences whether the species can track suitable conditions and relates to the species' dispersal ability. We approximated the dispersal ability of the avian frugivores by their wing pointedness measured on museum specimens. (c) A species' dietary niche breadth influences whether the species can tolerate shifts in food resources. For avian frugivores, this can be estimated by their bill width which influences the range of fruit sizes the species can feed on. (d) Similarly, a species' habitat niche breadth influences whether the species can tolerate shifts in 
available habitat. We approximated the habitat niche breadth of the avian frugivores as the number of habitat classes the species are reported to occur in. The illustration of avian wing morphology is adapted from Sheard et al. (2020).

\section{Methods}

\section{Study system and assemblages of frugivorous birds}

We studied 215 frugivorous bird species at twelve elevational levels located every 300 $\mathrm{m}$ from 300 to $3600 \mathrm{~m}$ a.s.I. in the Manú biosphere reserve in southeast Peru. The elevational gradient is covered in lowland rainforest ( $<500 \mathrm{~m}$ a.s.I.), montane rainforest ( $\sim 500$ to $1500 \mathrm{~m}$ a.s.I.), cloud forest ( 1500 to $3000 \mathrm{~m}$ a.s.l) and elfin forest (> $3000 \mathrm{~m}$ a.s.I.). At the treeline ( $\sim 3500 \mathrm{~m}$ a.s.I.), elfin forest is interrupted by patches of Puna grassland (Patterson et al. 1998). Precipitation is high along the entire gradient (annual rainfall approximately $1500-4800 \mathrm{~mm}$, mean $=2709 \mathrm{~mm}$ ) while temperature declines with increasing elevation (mean annual temperature ranges from $24.3^{\circ} \mathrm{C}$ at $500 \mathrm{~m}$ a.s.l. to $7.3^{\circ} \mathrm{C}$ at $3500 \mathrm{~m}$ a.s.l.; Girardin et al. 2010, 2013).

We determined the local elevational ranges (i.e., minimum and maximum elevation) of the 215 frugivorous bird species based on local checklists (Walker et al. 2006, Merkord 2010, Dehling et al. 2013, 2014b). The species pool initially included all frugivorous species reported in the region ( $n=245$ species). However, to ensure unbiased estimates of species' climatic niches (see description below), we excluded species with strong seasonal migrations (Elaenia albiceps chilensis, Elaenia parvirostris, Elaenia spectabilis, Tyrannus savanna, all subspecies of Thraupis bonariensis except subspecies darwinii). Furthermore, we excluded ground-dwelling species (the families Tinamidae, Odontophoridae, Psophidae, and the genus Mitu), because their dispersal behaviour does not match our proxy for dispersal ability (see description below).

\section{Sensitivity: climatic niche breadth}

We estimated species' climatic niche breadth based on bioclimatic variables and species' occurrences across South America, i.e., we quantified species' realized climatic niche breadth across the entire continent (Fig. 1 a). We downloaded occurrence data for each bird species from the Global Biodiversity Information Facility (GBIF.org 2017) and subjected the data to a comprehensive quality check. First, we excluded data entries with a longitude of zero and data entries for which the country provided by the author of the data did not resemble the country in which the 
coordinates were located. Second, we compared the GBIF occurrences to species' extent-of-occurrence range maps (BirdLife International and Handbook of the Birds of the World 2017) and removed outliers, i.e., occurrence points $>500 \mathrm{~km}$ away from the range map margins. Moreover, we only analysed species for which at least 20 spatially unique occurrence points were available (every latitude-longitude combination was counted once, even if several occurrences have been recorded for the same location) and where these points covered the species' range maps reasonably well. The final set of 215 bird species had on average $437 \pm 431$ spatially unique occurrence points (mean $\pm \mathrm{sd}$ ) ranging from 24 to 3467 spatially unique occurrence points per species. Only eleven of these species had less than 50 spatially unique occurrence points and those eleven species were mostly small-ranged.

We downloaded current bioclimatic data (1979-2013) from the Climatologies at High resolution for the Earth's Land Surface Areas data (CHELSA; Karger et al. 2017) at a resolution of 30 arcsec. We selected 17 bioclimatic variables that capture minimum, maximum, and mean values as well as diurnal and seasonal variation of temperature and precipitation across South America (Table S1, Supporting information). The CHELSA data have the advantage of including orographic predictors in the precipitation estimation, thereby enhancing rainfall estimates based on interpolated weather station data, especially in mountainous regions (Karger et al. 2017).

Using the cleaned set of occurrence points and the bioclimatic variables, we estimated each species' climatic niche breadth as a two-dimensional hypervolume following Blonder et al. (2014). To reduce the number of dimensions before the computation of the climatic niche breadth of each species (as suggested by Blonder et al. 2014), we performed a principal component analysis (PCA) across the values of the 17 bioclimatic variables at all occurrence points of the 215 bird species across South America. We included each latitude-longitude combination only once, even if several species were reported to occur at the same location. We used the first two axes of this PCA (capturing $76.59 \%$ of the total variation in the occurrence data, Table S1, Supporting information) and computed each species' climatic niche breadth as a twodimensional hypervolume in this PCA space (Blonder et al. 2014). The first principal component was positively correlated with bioclimatic variables related to mean annual temperature and annual precipitation and negatively correlated with seasonality in temperature and precipitation and with mean diurnal range. The second principal 
component was positively correlated with variables related to annual precipitation and negatively correlated with variables related to mean annual temperature, seasonality in temperature and precipitation, and with mean diurnal range (Table S1, Supporting information). The hypervolume function performs a kernel density estimation and volume measurement using a Monte Carlo importance sampling approach (Blonder et al. 2014). We applied Gaussian kernel density and Silverman bandwidth estimation (default settings in $\mathrm{R}$ package 'hypervolume'). To test whether the two-dimensional hypervolume is a robust estimate of species' climatic niche breadth, we compared it to the estimates based on three- and four-dimensional hypervolumes (including the first three and four principal component axes; Table S1, Supporting information) and to estimates based on a method suggested by Broennimann et al. (2012; details in Supporting information). The estimates of species' climatic niche breadth based on these different approaches were positively correlated (Pearson's $r$ ranging from 0.54 to $0.92, p<0.001$, Table S2, Supporting information).

\section{Adaptive capacity: dispersal ability, dietary niche breadth, and habitat niche breadth}

We estimated the dispersal ability and the dietary niche breadth of the 215 selected frugivorous bird species based on species' morphological traits, i.e., based on their wing pointedness and bill width (Fig. $1 \mathrm{~b}, \mathrm{c}$ ). The wing pointedness of bird species is related to their natal dispersal distances and their capacity to fly long distances (Winkler and Leisler 1992, Dawideit et al. 2009, Santini et al. 2019). Therefore, measures of wing pointedness can serve as a general proxy for the dispersal ability of a bird species (Sheard et al. 2020). The dietary niche breadth of frugivorous birds can be approximated by species' bill width since broad-billed frugivorous species can feed on a wider range of fruit sizes than narrow-billed species and are therefore more flexible in their fruit choice (Wheelwright 1985). We focus on a trait related to fruit consumption since all species in our dataset consume fruits as a main part of their diet (Kissling et al. 2009, Dehling et al. 2014b). Wing pointedness and bill width for each species were measured on museum specimen following measurement protocols from Eck et al. (2011) aiming at measuring two female and two male specimens per species (dataset from Dehling et al. 2014b). Wing pointedness was measured as Kipp's index, which is the distance from the tip of the first secondary feather to the tip of the longest primary feather $(\mathrm{mm})$ divided by wing length $(\mathrm{mm}$; equivalent to the hand-wing index; 
Eck et al. 2011, Sheard et al. 2020). The average number of specimens measured per species was $3.6 \pm 0.9$ (mean $\pm \mathrm{sd}$ ). Only for three of the 215 bird species, measurements were based on one specimen. For all further analyses, we computed mean values of wing pointedness and bill width for each species (Table S3, Supporting information).

Estimates of habitat niche breadth for all 215 species were derived from habitat affiliations of terrestrial birds based on the habitat classification scheme (version 3) of the International Union for Conservation of Nature. We included eleven habitat classes representing a gradient from forest to open habitats (forest, plantations, savanna, shrubland, rural gardens, subtropical/tropical heavily degraded former forest, grassland, pastureland, arable land, desert, rocky areas). Habitat niche breadth was estimated as the number of habitat classes in which a species was recorded (dataset from Barnagaud et al. 2017) and reflects the difference between species that are spread across all or many habitats (generalists) and those restricted to a few habitats (specialists; Fig. 1 d).

\section{Relationships of species' sensitivity and adaptive capacity with elevation}

We assessed relationships of species' sensitivity and adaptive capacity with elevation by fourth-corner analyses. The fourth-corner analysis was developed to test for relationships between environmental variables (here elevation as a surrogate for changing abiotic and biotic conditions with elevation) and species' traits based on species' occurrences (here presence/absence of bird species at each of the 12 elevational levels, 300 to 3600 m a.s.I.; Dray and Legendre 2008). Specifically, the fourth-corner analysis assesses the link between a table containing the occurrence of species at sites, a table containing the measurements of environmental variables for the sites, and a table describing species traits. For continuous variables, the relationship is assessed as Pearson correlation coefficient. The significance of the relationship is tested with a permutation model. To avoid inflated type I error, we applied permutation model 6 (Dray and Legendre 2008). We performed a separate fourth-corner analysis for each of the traits and attributes. Since we expected saturating trends with increasing elevation, we log-transformed elevation prior to the analyses. When elevation was not log-transformed prior to the analysis, the fourthcorner analyses yielded similar results (Table S4, Supporting information). 


\section{Relationships of species' sensitivity and adaptive capacity across species}

We tested for associations among the response traits and attributes that quantify species' sensitivity and adaptive capacity across species with phylogenetic generalized least square models (PGLS). PGLS models take into account the phylogenetic covariance among species (Freckleton et al. 2002). We based the phylogenetic analyses on the global phylogeny for birds (Jetz et al. 2012; details in Supporting information). We applied PGLS models since bill width (lambda $=1.00, p=$ 0.001 ) and wing pointedness (lambda $=1.00, p=0.001$ ) showed significant phylogenetic signal under the assumption of trait evolution as a random walk under a constant evolutionary rate (Brownian Motion). For climate and habitat niche breadth, lambda was $0.35(p=0.001)$, and $0.30(p=0.006)$, respectively, suggesting a significant phylogenetic signal, but also that these attributes evolved according to a process in which the effect of the phylogeny was weaker than in the Brownian motion model (Freckleton et al. 2002). We fitted a PGLS model each for wing pointedness, bill width, and habitat niche breadth (as measures of a species' adaptive capacity) against climatic niche breadth (the sensitivity measure) to control for these phylogenetic signals. Since the habitat niche breadth is represented by count data (i.e., the number of inhabited habitat types), we log-transformed it before fitting the model. In these PGLS models, we set delta and kappa to one and estimated lambda by maximum likelihood. For wing pointedness, the model was not able to yield a maximum likelihood estimate for lambda due to a flat likelihood surface, so we set lambda to one in this model. All analyses were performed in R version 3.5.0 ( $R$ Core Team 2018). 


\section{Results}

\section{Relationships of species' sensitivity and adaptive capacity with elevation}

The fourth-corner analyses revealed a significant positive relationship between elevation and species' climatic niche breadth (Table 1). Specifically, species' climatic niche breadth increased until about $1200 \mathrm{~m}$ a.s.l. and showed only little change between 1200 and 3600 m a.s.l. (Fig. 2 a). In contrast, wing pointedness and bill width, approximating species' dispersal ability and dietary niche breadth, respectively, decreased significantly with increasing elevation (Table 1, Fig. 2 b, c). Species' habitat niche breadth showed no significant association with elevation (Table 1, Fig. 2 d). This suggests that species' sensitivity to climate change and their adaptive capacity in terms of dispersal ability and resource use decrease with increasing elevation.

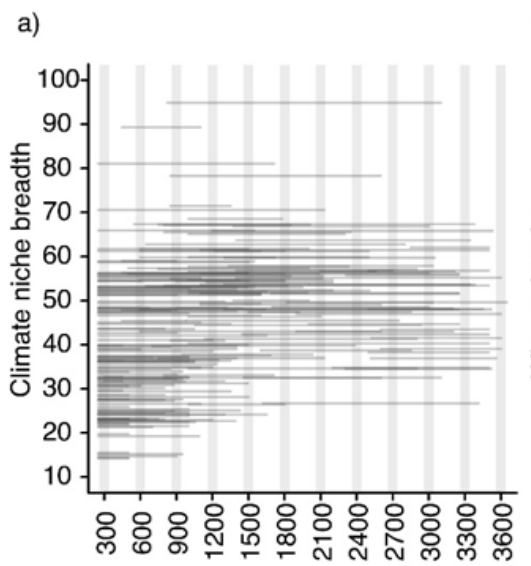

b)

c)

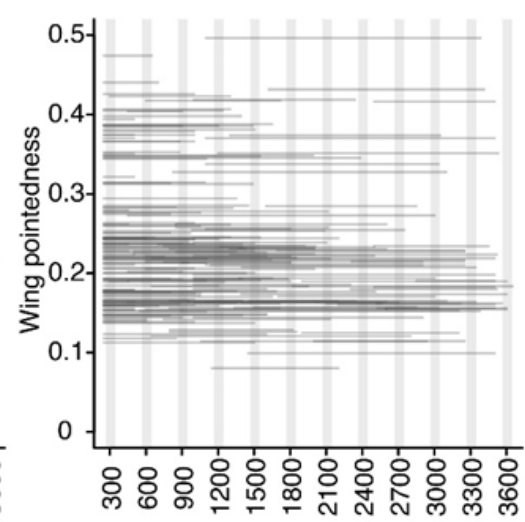

d)

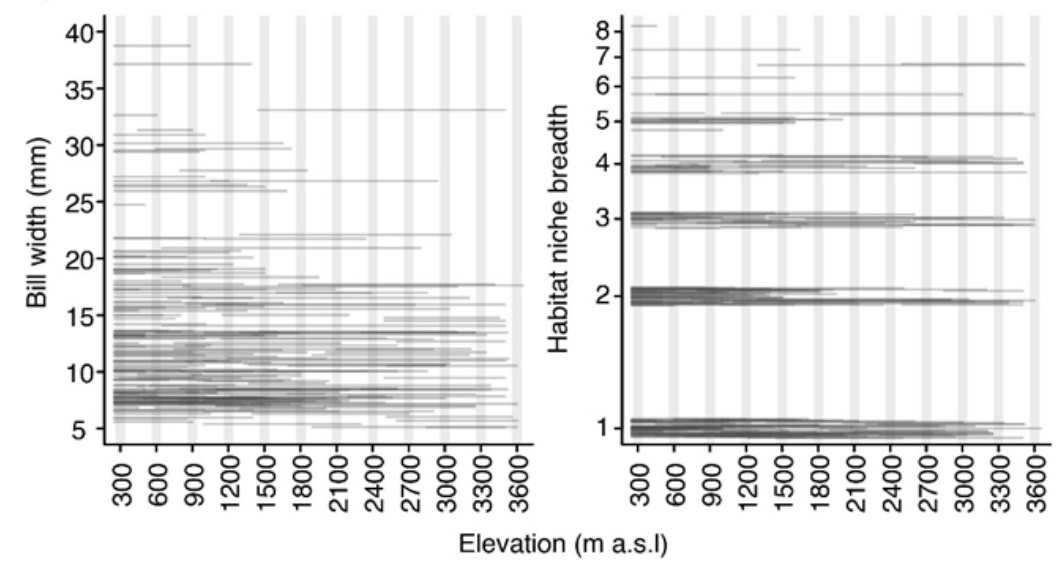

Figure 2: Elevational patterns of species' sensitivity to climate change (a) and their adaptive capacity (b-d). Shown are patterns of species' (a) climatic niche breadth, (b) wing pointedness (our proxy for species' dispersal ability) (c) bill width (our proxy for species' dietary niche breadth) and (d) habitat niche breadth (i.e., number of habitat types) along elevation. Horizontal lines represent each species' ( $n=215$ avian frugivores) elevational range in the study area (from minimum to maximum elevation). Vertical lines indicate the 12 species assemblages studied at the different elevations. 
Table 1: Relationships between elevation and response traits and attributes related to species' sensitivity (climatic niche breadth) to climate change and their adaptive capacity (wing pointedness, dietary niche breadth and habitat niche breadth). We performed a separate fourth corner analysis for each of the traits and attributes; this tests the relationships based on species' occurrences at 12 elevational levels every $300 \mathrm{~m}$ along the Manú gradient (300-3,600m a.s.I.). Elevation was log-transformed prior to the analyses. Given are the Pearson correlation coefficient $r$, the $p$-value.

\begin{tabular}{lll} 
Response variable & Pearson's $\boldsymbol{r}$ & $\boldsymbol{p}$-value \\
\hline Climatic niche breadth & 0.35 & 0.002 \\
Wing pointedness & -0.12 & 0.046 \\
Bill width & -0.12 & 0.046 \\
Habitat niche breadth & 0.03 & 0.598
\end{tabular}

\section{Relationships of species' sensitivity and adaptive capacity across species}

Across species, there were no significant relationships between traits and attributes that quantify species' sensitivity and adaptive capacity when accounting for phylogenetic signals in the traits and attributes (Table 2). Species varied widely in the traits related to adaptive capacity across the entire spectrum of species' climatic niche breadths (Fig. 3). This indicates that traits and attributes related to species' sensitivity and adaptive capacity vary independently across species.

Interestingly, different bird orders showed some distinction in their sensitivity and adaptive capacity (Fig. 3). For instance, the climatic niche breadth of Passeriformes (perching birds, $n=148$ species) and Trogoniformes (trogons, $n=9$ ) varied from narrow to broad. However, Passeriformes had round to moderately pointed wings and narrow to moderately broad bills, while Trogoniformes were characterized by pointed wings and moderate bill width. Contrastingly, Psittaciformes (parrots, $n=26$ ) and Piciformes (woodpeckers, $n=21$ ) displayed mostly narrow to moderately broad climatic niches. Yet, Psittaciformes were among the species with the most pointed wings, while Piciformes had more rounded wings. 


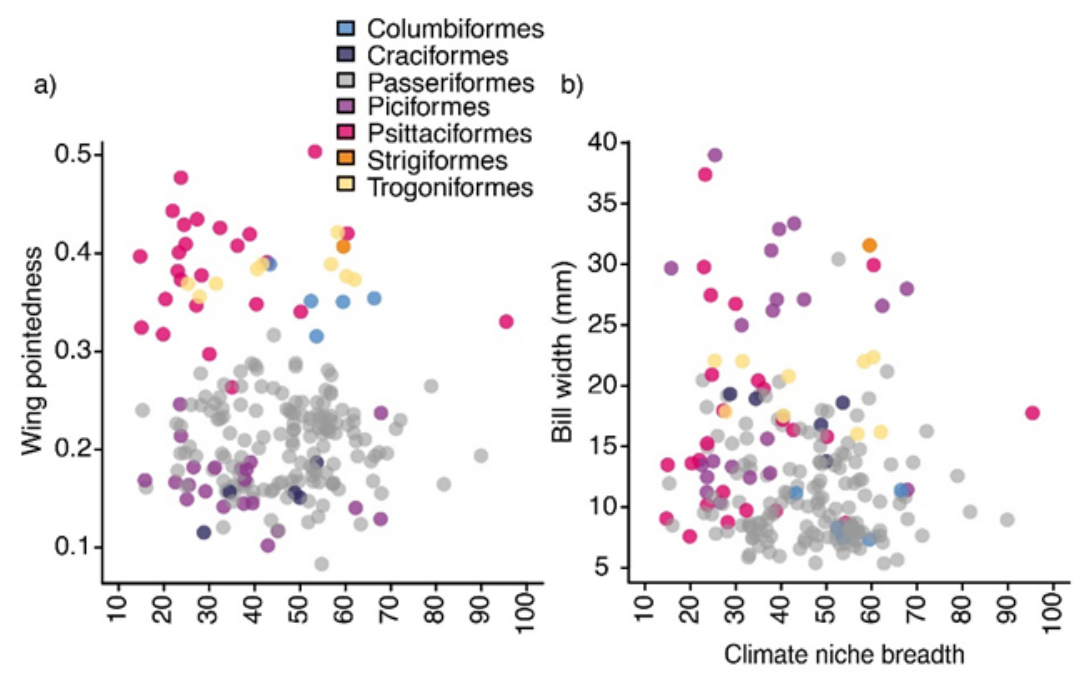

c)

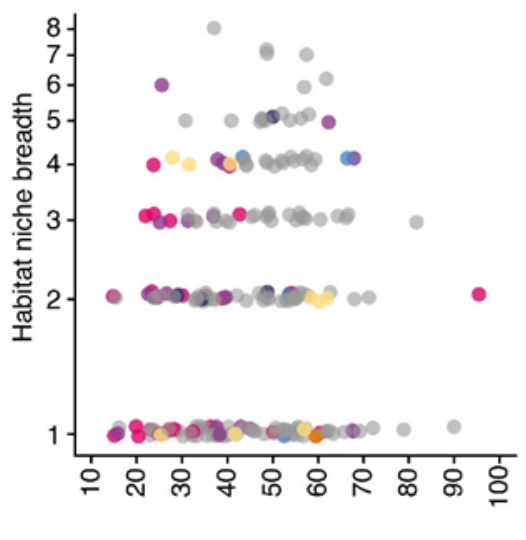

Figure 3: Associations of species' sensitivity to climate change (climatic niche breadth) with different aspects of their adaptive capacity. Shown are associations of climatic niche breadth and (a) wing pointedness (our proxy for species' dispersal ability), (b) bill width (our proxy for species' dietary niche breadth), and (c) habitat niche breadth (i.e. number of habitat types). Each dot represents a species $(n=215)$, colours represent the different orders of frugivorous birds.

Table 2: Results of phylogenetic generalized least square (pgls) models fitted to test for associations of species' sensitivity (climatic niche breadth) with aspects of their adaptive capacity (wing pointedness, bill width, habitat niche breadth) across species. All traits and attributes were scaled and centred before the analyses to ensure comparability of the model estimates. Given are the estimates for the intercept and the predictor, the standard error, $t$-value and $p$-value of the estimate. Adjusted $R^{2}$ of all models was $<0.01$.

\begin{tabular}{llcccr}
$\begin{array}{l}\text { Response } \\
\text { variable }\end{array}$ & $\begin{array}{l}\text { Intercept/ } \\
\text { Predictor }\end{array}$ & Estimate & $\begin{array}{r}\text { Std. } \\
\text { Error }\end{array}$ & t-value & $\boldsymbol{p}$-value \\
\hline Wing pointedness & Intercept & 0.33 & 0.56 & 0.59 & 0.56 \\
& Climatic niche breadth & 0.04 & 0.03 & 1.28 & 0.20 \\
& Intercept & 0.48 & 0.66 & 0.73 & 0.47 \\
Bill width & Climatic niche breadth & -0.02 & 0.03 & -0.67 & 0.50 \\
& & & & & \\
& Intercept & 0.04 & 0.37 & 0.10 & 0.92 \\
Habitat niche & Climatic niche breadth & 0.06 & 0.07 & 0.87 & 0.38 \\
breadth & & & & &
\end{tabular}




\section{Discussion}

We tested how species' sensitivity to climate change and ecological aspects of their adaptive capacity vary along an elevational gradient and covary across species. We found that species' climatic niche breadth increased with increasing elevation, while dispersal ability and dietary niche breadth decreased with increasing elevation. This indicates that the sensitivity of avian frugivore species to climate change might be highest in lowland assemblages, while highland assemblages host avian frugivore species that might have a comparatively low adaptive capacity in terms of their dispersal ability and resource use. Species' sensitivity to climate change and their adaptive capacity varied independently across species.

\section{Relationships of species' sensitivity and adaptive capacity with elevation}

As expected, we found a positive relationship between species' climatic niche breadth and elevation. This relationship is consistent with Rapoport's rule according to which species' range sizes increase with increasing elevation because of a greater tolerance for climatic variation in highland species (Stevens 1989, 1992). Along the Manú gradient, increasing diurnal temperature variation with elevation might favour avian frugivore species with broader thermal tolerances at higher elevations (Ghalambor et al. 2006, Rapp and Silman 2012). Our finding is in accordance with the climate variability hypothesis and the positive relationship of avian thermal niche breadth with climate variability at global scale (Janzen 1967, Ghalambor et al. 2006, Khaliq et al. 2014). Indeed, high-elevation bird species in the Peruvian Andes have been reported to be more resistant to heat loss, but equally capable to withstand high lowland temperatures compared to species from lower elevations (Londoño et al. 2015). The Glossy-black Thrush (Turdus serranus) and the Hooded Mountain Tanager (Buthraupis montana) are examples for high-elevation species at the Manú gradient with rather broad climatic niches. Among the lowland-species with more narrow climatic niches are the Purple-throated Cotinga (Porphyrolaema porphyrolaema) and the Opal-rumped Tanager (Tangara velia).

The negative relationships between wing pointedness and bill width with elevation were in line with our expectations. Many tropical avian frugivore species are highly dependent on fruit in their diet (Kissling et al. 2009). Therefore, the significant but weak relationships we found might be due to bottom-up effects of the fruit plant assemblages on the avian frugivores and their traits (Vollstädt et al. 2017). Specifically, low plant 
heights and small fruit sizes at high elevations of the Manú gradient might promote round-winged and narrow-billed avian frugivores at these elevations (Dehling et al. 2014a, Pigot et al. 2016). This likely relates to trait matching between interacting resource and consumer species, specifically the positive relationship between wing pointedness and plant height in plant-frugivore interactions (Dehling et al. 2014a, Bender et al. 2018). Similarly, bill width and fruit width of interacting avian frugivore and fruit plant species usually correspond closely (Burns 2013, Bender et al. 2018). The decreasing availability of fruit resources with increasing elevation might lead to environmental filtering of bird traits resulting in low trait diversity of high-elevation bird assemblages (Hanz et al. 2019). Among the high-elevation species with rather round wings and narrow bills are the Blue-capped Tanager (Thraupis cyanocephala) and the Blue-winged Mountain Tanager (Anisognathus somptuosus), while the Red-bellied Macaw (Orthopsittaca manilata) is an example for a pointed-winged, broad-billed lowland species.

The opposing elevational patterns of climatic niche breadth on the one hand and dispersal ability and dietary niche breadth on the other hand suggest different mechanisms of how species from low vs. high-elevation assemblages might respond to contemporary climate change. Given their rather narrow climatic niches, avian frugivore species from lowland assemblages might be sensitive to climate change. However, occurrence-derived thermal tolerances of tropical lowland species might underestimate their actual thermal tolerances, since species might tolerate higher temperatures than currently realized (Feeley and Silman 2010). Still, lowland species will likely shift their elevational ranges upslope to track their suitable climate. Upslope range shifts in response to contemporary climate change have indeed been reported for bird species at tropical elevational gradients (Forero-Medina et al. 2011, Freeman and Class Freeman 2014). However, thus far tropical lowland bird species tend to expand their elevational ranges upslope under contemporary climate change suggesting that they are able to tolerate increasing temperatures, at least to some extent, despite their narrow realized climatic niches (Freeman et al. 2018a). The observed range expansion of lowland bird species is in line with the high dispersal ability of many lowland frugivores. Specifically, the pointed-winged species of the lowland assemblages might be well equipped for range shifts due to their good dispersal ability (Dawideit et al. 2009). Furthermore, the flexibility in fruit choice of broad-billed lowland frugivores might enhance their ability to find matching fruit 
resources at higher elevations (Wheelwright 1985). However, changes in the fruit resource use of avian frugivores need to be explored further (Bender et al. 2017).

The comparatively broad climatic niches of avian frugivores in assemblages at higher elevations of the Manú gradient might make these species less sensitive to climate change than species from low elevations. Accordingly, avian frugivore species at high elevations in the tropics were predicted to persist in their current assemblages despite projected future changes in precipitation and temperature (Bender et al. 2019). Moreover, a recent meta-analysis revealed that high-elevation species globally shifted their elevational ranges upslope at a lower rate than low-elevation species under contemporary climate change, which might be attributed to broader climatic niches of high-elevation species (Mamantov et al. 2021). However, high-elevation frugivores at the Manú gradient might be less well equipped to cope with future changes in the availability of fruit resources because their narrow bills restrict them to feed on rather small fruits (Moermond and Denslow 1985, Wheelwright 1985). Moreover, due to their round wings, they tend to be better equipped to forage in lower forest strata than in the canopy (Moermond and Denslow 1985, Dehling et al. 2014a). Yet, plants along tropical mountains are shifting their elevational ranges upslope under contemporary climate change, and this trend might continue in the future (Feeley et al. 2011, Morueta-Holme et al. 2015). Upslope range shifts of large-fruited and tall plant species from low elevations have been predicted to increase the average fruit size and plant height of fruit plant assemblages at higher elevations (Nowak et al. 2019). Hence, avian frugivore species currently residing at high elevations might not be able to efficiently utilize the fruit resources provided by future plant assemblages at these elevations (Fleming 1979, Dehling et al. 2014a).

Furthermore, avian frugivore assemblages at the highest elevations are restricted by the treeline. Therefore, species occurring close to this biotic barrier might contract their elevation ranges because they cannot easily expand their range to higher elevations (La Sorte and Jetz 2010). This phenomenon of so-called mountaintop extinctions has been widely reported and suggests that highland species face an elevated risk from rapid global warming (Pacifici et al. 2017, Freeman et al. 2018b). Range contractions towards the mountaintop have also been reported for highelevation bird species under recent climate change (Freeman et al. 2018a). To track future shifts in their suitable climate, high-elevation species would need to disperse to 
other mountain ranges or higher latitudes. Yet, due to their rather round wings, they might not be well equipped to disperse over such long distances (Dawideit et al. 2009).

\section{Relationships of species' sensitivity and adaptive capacity across species}

Contrary to our expectation, response traits and attributes related to the sensitivity of species to climate change and their adaptive capacity were unrelated across the avian frugivore species at the Manú gradient. Accordingly, we could not identify trait syndromes of species with coherent patterns in terms of their sensitivity and adaptive capacity. This was confirmed by the variation in trait values within bird orders, e.g., the perching birds. Generally, some species with pointed wings and broad bills were characterized by broad climatic niches, e.g., the Golden-Headed Quetzal (Pharomachrus auriceps) and the Military Macaw (Ara militaris). Other species had broad climatic niches, but round wings or narrow bills, e.g., the Bronze-Green Euphonia (Euphonia mesochrysa) and the Spotted Tanager (Tangara punctata). Some species with narrow climatic niches had pointed wings or broad bills, e.g., the BlackTailed Trogon (Trogon melanurus) and the Green-Backed Trogon (Trogon viridis). These species-specific differences of sensitivity and adaptive capacity within the group of avian frugivores coincide with the observation that individual species can differ greatly in their responses to climate change (Moritz et al. 2008, Chen et al. 2011). Our findings reveal that even within ecologically homogeneous groups of species, responses to climate change are likely to be individualistic.

Interestingly, our findings differ from a global study reporting increasing average wing pointedness with increasing climate variability (Sheard et al. 2020). Furthermore, we could not confirm a positive or negative relationship between climatic niche breadth and habitat niche breadth across avian species (Barnagaud et al. 2012, Reif et al. 2016). Hence, relationships of species' sensitivity and adaptive capacity might be context-dependent. Specifically, this relationship appears to differ between global and local scales, and when including migratory species in the comparison (Sheard et al. 2020). Furthermore, the relationship seems to differ between tropical avian frugivore assemblages with a high trait diversity compared to the less diverse European bird assemblages (Kissling et al. 2009, Barnagaud et al. 2012, Reif et al. 2016). Although we did not detect a systematic relationship between sensitivity and adaptive capacity across species, we encourage future studies exploring whether such relationships 
exist with aspects of species' adaptive capacity not included here, e.g., the evolutionary potential of species (Boutin and Lane 2014).

\section{Conclusions}

We show that response traits and attributes related to the sensitivity of avian frugivores to climate change and their adaptive capacity vary independently along elevation and across species. Our results emphasize that focussing on the sensitivity of species to climate change (i.e., their climatic niche) can be insufficient to predict potential effects of future climate change on species assemblages in the tropics. While trait-based approaches can provide a ready way to assess the potential effects of future climate change on species assemblages, they should consider different dimensions of species' susceptibility to climate change.

\section{References}

Algar, A. C. and Tarr, S. 2018. Fossils, phylogenies and the evolving climate niche. Nat. Ecol. Evol. 2: 414-415.

Barnagaud, J.-Y. et al. 2012. Relating habitat and climatic niches in birds. - PLoS One 7: e32819.

Barnagaud, J. Y. et al. 2017. Biogeographical, environmental and anthropogenic determinants of global patterns in bird taxonomic and trait turnover. - Glob. Ecol. Biogeogr. 26: 1190-1200.

Bellard, C. et al. 2012. Impacts of climate change on the future of biodiversity. - Ecol. Lett. 15: 365-377.

Bender, I. M. A. et al. 2017. Functionally specialised birds respond flexibly to seasonal changes in fruit availability. - J. Anim. Ecol. 86: 800-811.

Bender, I. M. A. et al. 2018. Morphological trait matching shapes plant-frugivore networks across the Andes. - Ecography 41: 1-10.

Bender, I. M. A. et al. 2019. Projected impacts of climate change on functional diversity of frugivorous birds along a tropical elevational gradient. - Sci. Rep. 9: 17708.

BirdLife International and Handbook of the Birds of the World 2017. Bird species distribution maps of the world. Version 2017.2.

Blonder, B. et al. 2014. The n-dimensional hypervolume. - Glob. Ecol. Biogeogr. 23: 595-609. 
Botts, E. A. et al. 2013. Small range size and narrow niche breadth predict range contractions in South African frogs. - Glob. Ecol. Biogeogr. 22: 567-576.

Boutin, S. and Lane, J. E. 2014. Climate change and mammals: evolutionary versus plastic responses. - Evol. Appl. 7: 29-41.

Broennimann, O. et al. 2012. Measuring ecological niche overlap from occurrence and spatial environmental data. - Glob. Ecol. Biogeogr. 21: 481-497.

Burns, K. C. 2013. What causes size coupling in fruit-frugivore interaction webs? Ecology 94: 295-300.

Chen, I. C. et al. 2011. Rapid range shifts of species associated with high levels of climate warming. - Science 333: 1024-1026.

Culp, L. A. et al. 2017. Full annual cycle: climate change vulnerability assessment for migratory birds. - Ecosphere 8: e01565.

Dawideit, B. A. et al. 2009. Ecomorphological predictors of natal dispersal distances in birds. - J. Anim. Ecol. 78: 388-395.

Dehling, D. M. et al. 2013. Upper and lower elevational extremes of Andean birds from south-east Peru. - Boletín Inf. 8: 32-38.

Dehling, D. M. et al. 2014a. Functional relationships beyond species richness patterns: trait matching in plant-bird mutualisms across scales. - Glob. Ecol. Biogeogr. 23: 1085-1093.

Dehling, D. M. et al. 2014b. Functional and phylogenetic diversity and assemblage structure of frugivorous birds along an elevational gradient in the tropical Andes. - Ecography 37: 1047-1055.

Di Musciano, M. et al. 2020. Dispersal ability of threatened species affects future distributions. - Plant Ecol. 221: 265-281.

Dray, S. and Legendre, P. 2008. Testing the species traits environment relationships: the fourth-corner problem revisited. - Ecology 89: 3400-3412.

Eck, S. et al. 2011. Measuring birds. - Christ Media Natur.

Fang, M. et al. 2019. Macroecological patterns of climatic niche breadth variation in lacertid lizards. - Asian Herpetol. Res. 10: 41-47.

Feeley, K. J. and Silman, M. R. 2010. Biotic attrition from tropical forests correcting for truncated temperature niches. - Glob. Chang. Biol. 16: 1830-1836.

Feeley, K. J. et al. 2011. Upslope migration of Andean trees. - J. Biogeogr. 38: 783791. 
Fleming, T. H. 1979. Do tropical frugivores compete for food? - Am. Zool. 19: 11571172.

Foden, W. B. et al. 2013. Identifying the world's most climate change vulnerable species: a systematic trait-based assessment of all birds, amphibians and corals. - PLoS One 8: e65427.

Foden, W. B. et al. 2018. Climate change vulnerability assessment of species. - Wiley Interdiscip. Rev. Clim. Chang. 10: e551.

Forero-Medina, G. et al. 2011. Elevational ranges of birds on a tropical montane gradient lag behind warming temperatures. - PLoS One 6: e28535.

Freckleton, R. P. et al. 2002. Phylogenetic analysis and comparative data: a test and review of evidence. - Am. Nat. 160: 712-726.

Freeman, B. G. and Class Freeman, A. M. 2014. Rapid upslope shifts in New Guinean birds illustrate strong distributional responses of tropical montane species to global warming. - Proc. Natl. Acad. Sci. 111: 4490-4494.

Freeman, B. G. et al. 2018a. Climate change causes upslope shifts and mountaintop extirpations in a tropical bird community. - Proc. Natl. Acad. Sci. 115: 1198211987.

Freeman, B. G. et al. 2018b. Expanding, shifting and shrinking: the impact of global warming on species' elevational distributions. - Glob. Ecol. Biogeogr. 27: 12681276.

GBIF.org 2017. GBIF occurence download.

Ghalambor, C. K. et al. 2006. Are mountain passes higher in the tropics? janzen's hypothesis revisited. - Integr. Comp. Biol. 46: 5-17.

Girardin, C. A. J. et al. 2010. Net primary productivity allocation and cycling of carbon along a tropical forest elevational transect in the Peruvian Andes. - Glob. Chang. Biol. 16: 3176-3192.

Girardin, C. A. J. et al. 2013. Fine root dynamics along an elevational gradient in tropical Amazonian and Andean forests. - Global Biogeochem. Cycles 27: 252264.

Hanz, D. M. et al. 2019. Functional and phylogenetic diversity of bird assemblages are filtered by different biotic factors on tropical mountains. - J. Biogeogr. 46: 291303. 
Herrera, J. M. et al. 2018. Climatic niche breadth determines the response of bumblebees (Bombus spp.) to climate warming in mountain areas of the Northern Iberian Peninsula. - J. Insect Conserv. 22: 771-779.

IPCC 2007. Climate Change 2007: synthesis report. Contribution of working groups I, II and III to the fourth assessment report of the Intergovernmental Panel on Climate Change [Core Writing Team, Pachauri, R.K and Reisinger, A. (eds.)]. IPCC 2014. Climate change 2014: synthesis report. Contribution of working groups I, II and III to the fifth assessment report of the Intergovernmental Panel on Climate Change [Core Writing Team, R.K. Pachauri and L.A. Meyer (eds.)].

Jackson, M. M. et al. 2015. Effects of climate change on habitat availability and configuration for an endemic coastal alpine bird. - PLoS One 11: e0142110.

Janzen, D. H. 1967. Why mountain passes are higher in the tropics. - Am. Nat. 101: 233-249.

Jetz, W. et al. 2012. The global diversity of birds in space and time. - Nature 491: 444448.

Karger, D. N. et al. 2017. Climatologies at high resolution for the earth's land surface areas. - Sci. Data 4: 1-20.

Khaliq, I. et al. 2014. Global variation in thermal tolerances and vulnerability of endotherms to climate change. - Proc. R. Soc. B Biol. Sci. 281: 20141097.

Kissling, D. W. et al. 2009. The global distribution of frugivory in birds. - Glob. Ecol. Biogeogr. 18: 150-162.

La Sorte, F. A. and Jetz, W. 2010. Projected range contractions of montane biodiversity under global warming. - Proc. R. Soc. B Biol. Sci. 277: 3401-3410.

Lenoir, J. et al. 2020. Species better track climate warming in the oceans than on land. - Nat. Ecol. Evol. 4: 1044-1059.

Londoño, G. A. et al. 2015. Basal metabolism in tropical birds: latitude, altitude, and the "pace of life." - Funct. Ecol. 29: 338-346.

Luck, G. W. et al. 2012. Improving the application of vertebrate trait-based frameworks to the study of ecosystem services. - J. Anim. Ecol. 81: 1065-1076.

Mamantov, M. A. et al. 2021. Climate-driven range shifts of montane species vary with elevation. - Glob. Ecol. Biogeogr. 00: 1-11.

Maron, M. et al. 2015. Climate-induced resource bottlenecks exacerbate species vulnerability: a review. - Divers. Distrib. 21: 731-743. 
Merkord, C. L. 2010. Seasonality and elevational migration in an Andean bird community.

Moermond, T. C. and Denslow, J. S. 1985. Neotropical avian frugivores : patterns of behavior, morphology, and nutrition, with consequences for fruit selection. Ornithol. Monogr. 36: 865-897.

Moritz, C. et al. 2008. Impact of a century of climate change on small-mammal communities in Yosemite National Park, USA. - Science 322: 261-264.

Morueta-Holme, N. et al. 2015. Strong upslope shifts in Chimborazo's vegetation over two centuries since Humboldt. - Proc. Natl. Acad. Sci. 112: 12741-12745.

Nowak, L. et al. 2019. Projecting consequences of global warming for the functional diversity of fleshy-fruited plants and frugivorous birds along a tropical elevational gradient. - Divers. Distrib. 25: 1362-1374.

Nunez, S. et al. 2019. Assessing the impacts of climate change on biodiversity: is below $2{ }^{\circ} \mathrm{C}$ enough? - Clim. Change 154: 351-365.

Pacifici, M. et al. 2015. Assessing species vulnerability to climate change. - Nat. Clim. Chang. 5: 215-224.

Pacifici, M. et al. 2017. Species' traits influenced their response to recent climate change. - Nat. Clim. Chang. 7: 205-208.

Patterson, B. D. et al. 1998. Contrasting patterns of elevational zonation for birds and mammals in the Andes of southeastern Peru. - J. Biogeogr. 25: 593-607.

Pigot, A. L. et al. 2016. Functional traits reveal the expansion and packing of ecological niche space underlying an elevational diversity gradient in passerine birds. - Proc. R. Soc. B Biol. Sci. 283: 20152013.

R Core Team 2018. R: a language and environment for statistical computing. in press.

Rapp, J. M. and Silman, M. R. 2012. Diurnal, seasonal, and altitudinal trends in microclimate across a tropical montane cloud forest. - Clim. Res. 55: 17-32.

Razgour, O. et al. 2019. Considering adaptive genetic variation in climate change vulnerability assessment reduces species range loss projections. - Proc. Natl. Acad. Sci. U. S. A. 116: 10418-10423.

Reif, J. et al. 2016. Linking habitat specialization with species' traits in European birds. - Oikos 125: 405-413.

Santini, L. et al. 2019. Applying habitat and population-density models to land-cover time series to inform IUCN Red List assessments. - Conserv. Biol. 33: 10841093. 
Schleuning, M. et al. 2012. Specialization of mutualistic interaction networks decreases toward Tropical latitudes. - Curr. Biol. 22: 1925-1931.

Schloss, C. A. et al. 2012. Dispersal will limit ability of mammals to track climate change in the Western Hemisphere. - Proc. Natl. Acad. Sci. 109: 8606-8611.

Shah, A. A. et al. 2017. Climate variability predicts thermal limits of aquatic insects across elevation and latitude. - Funct. Ecol. 31: 2118-2127.

Sheard, C. et al. 2020. Ecological drivers of global gradients in avian dispersal inferred from wing morphology. - Nat. Commun. 11: 1-9.

Slatyer, R. A. et al. 2013. Niche breadth predicts geographical range size: a general ecological pattern. - Ecol. Lett. 16: 1104-1114.

Stevens, G. C. 1989. The latitudinal gradient in geographical range: how so many species coexist in the tropics. - Am. Nat. 133: 240-256.

Stevens, G. C. 1992. The elevational gradient in altitudinal range: an extension of Rapoport's latitudinal rule to altitude. - Am. Nat. 140: 893-911.

Sunday, J. M. et al. 2015. Species traits and climate velocity explain geographic range shifts in an ocean-warming hotspot. - Ecol. Lett. 18: 944-953.

Thuiller, W. et al. 2006. Vulnerability of African mammals to anthropogenic climate change under conservative land transformation assumptions. - Glob. Chang. Biol. 12: 424-440.

Vázquez, D. P. and Stevens, R. D. 2004. The latitudinal gradient in niche breadth: concepts and evidence. - Am. Nat. 164: E1-E19.

Vollstädt, M. G. R. et al. 2017. Direct and indirect effects of climate, human disturbance and plant traits on avian functional diversity. - Glob. Ecol. Biogeogr. 26: 963-972.

Walker, B. et al. 2006. Birds of the Manu Biosphere Reserve. - Fieldiana Zool.: 23-49.

Wheelwright, N. T. 1985. Fruit-size, gape width, and the diets of fruit-eating birds. Ecology 66: 808-818.

Winkler, H. and Leisler, B. 1992. On the ecomorphology of migrants. - Ibis (Lond. 1859). 134: 21-28. 


\section{Supporting information}

\section{Methods}

\section{S1: Phylogenetic analyses}

We based the phylogenetic analyses on the global phylogeny for birds (Jetz et al. 2012). We downloaded a subset of 1000 posterior trees for our 215 bird species from the global avian phylogeny at BirdTree.org (2019) selecting the version V2.iii (i.e. Stage 2: MayrAll Hacket). For further analyses, we created a consensus tree with the software TreeAnnotator (Bouckaert et al. 2019).

\section{S2: Climatic niche breadth following Broennimann et al. (2012)}

The method recommended by Broennimann et al. (2012) defines species' climatic niche breadth in a two-dimensional climate space. Hence we utilized the first two axes from a principal component analysis carried out across all 215 bird species considering unique occurrence points and 17 bioclimatic variables (see main manuscript). The twodimensional climate space was gridded (100 x 100 cells) and for each species a value of occupancy in each of the cells in the climate space was computed. To this end, kernel density smoothing was used to generate density surfaces of the available climate and species occurrences and species' occupancy was estimated as occurrence density divided by climate density (Broennimann et al. 2012). Species climatic niche breadth was then estimated as Shannon diversity index accounting for the number of cells a species occupies in the climate space and the evenness of species' occupancy (Laube et al. 2015, Eyres et al. 2020). These analyses were performed with the R packages 'ecospat' and 'vegan'. 


\section{Tables}

Table S1: Bioclimatic variables and their correlations with principal component (PC) axes. Given are the 17 bioclimatic variables considered in our study and their correlation with the first, second, third and fourth PC axis. Further given is the amount of variance in the bird species' occurrences explained by the respective PC axis. The first and second PC axis (highlighted in grey) were utilized to estimate species' climatic niche as two-dimensional hypervolume in the main text (see table S2 for consideration of more dimensions).

\begin{tabular}{lrrrr}
\hline Bioclimatic variable & PC1 & PC2 & PC3 & PC4 \\
\hline Minimum Temperature of Coldest Month & 0.90 & -0.38 & -0.23 & 0.01 \\
\hline Mean Temperature of Driest Quarter & 0.88 & -0.44 & -0.13 & 0.00 \\
\hline Mean Temperature of Coldest Quarter & 0.88 & -0.44 & -0.16 & 0.02 \\
\hline Annual Mean Temperature & 0.85 & -0.52 & 0.04 & -0.02 \\
\hline Mean Temperature of Warmest Quarter & 0.80 & -0.57 & 0.18 & -0.05 \\
\hline Mean Temperature of Wettest Quarter & 0.79 & -0.57 & 0.15 & -0.03 \\
\hline Annual Precipitation & 0.79 & 0.58 & 0.14 & 0.11 \\
\hline Maximum Temperature of Warmest Month & 0.76 & -0.59 & 0.24 & -0.07 \\
\hline Precipitation of Wettest Quarter & 0.75 & 0.48 & 0.17 & 0.38 \\
\hline Precipitation of Wettest Month & 0.74 & 0.49 & 0.18 & 0.38 \\
\hline Precipitation of Coldest Quarter & 0.68 & 0.43 & -0.03 & 0.03 \\
\hline Precipitation of Driest Quarter & 0.67 & 0.61 & 0.09 & -0.32 \\
\hline Precipitation of Driest Month & 0.67 & 0.60 & 0.10 & -0.34 \\
\hline Precipitation of Warmest Quarter & 0.45 & 0.63 & 0.28 & 0.16 \\
\hline Temperature Seasonality & -0.24 & -0.30 & 0.83 & -0.18 \\
\hline Precipitation Seasonality & -0.45 & -0.46 & 0.15 & 0.64 \\
\hline Mean Diurnal Range & -0.58 & -0.12 & 0.62 & -0.06 \\
\hline & & & & \\
\hline Amount of variance explained (\%) & 51.7 & 24.89 & 8.78 & 5.85 \\
\hline
\end{tabular}

Table S2: Pearson correlation coefficients between different estimates of species' climatic niche breadth. We computed and contrasted climatic niche breadth estimated with two-, three- and four-dimensional hypervolumes (2d-, 3d- and 4d-hypervolume) based on the first two, three, or four climatic PC axes (Table S1), respectively, following Blonder et al (2014), and climatic niche breadth estimated with kernel density smoothing in two-dimensional climate space based on the first two climatic PC axes (see supplementary methods) following Broennimann et al. (2012). p-value $<0.001$ for all correlation tests.

\begin{tabular}{llll}
\hline & 2d-hypervoulme & 3d-hypervolume & 4d-hypervolume \\
\hline Broennimann & 0.67 & 0.68 & 0.54 \\
\hline 2d-hypervolume & & 0.79 & 0.71 \\
\hline 3d-hypervolume & & & 0.92 \\
\hline
\end{tabular}


Table S3: Response traits and number of occurrence points for 215 avian frugivores considered in our study. Table provided as separate excel file.

Table S4: Relationships between elevation (not transformed) and response traits or attributes related to species' sensitivity to climate change and to their adaptive capacity. We performed a separate fourth corner analysis for each of the traits and attributes; this tests the relationships based on species' occurrences at 12 elevational levels every $300 \mathrm{~m}$ along the Manú gradient (300-3,600m a.s.l.). Given are the Pearson correlation coefficient $r$, the $p$-value, and the significance level for the respective relationship.

\begin{tabular}{lll}
\hline Test & Pearson's $\boldsymbol{r}$ & $\boldsymbol{p}$-value \\
\hline Climatic niche breadth & 0.29 & 0.024 \\
\hline Wing pointedness & -0.11 & 0.066 \\
\hline Bill width & -0.11 & 0.070 \\
\hline Habitat niche breadth & 0.05 & 0.453 \\
\hline
\end{tabular}

\section{References}

BirdTree.org. (2019). BirdTree.org. Available at: http://birdtree.org/. Last accessed .

Blonder, B., Lamanna, C., Violle, C. \& Enquist, B.J. (2014). The n-dimensional hypervolume. Glob. Ecol. Biogeogr., 23, 595-609.

Bouckaert, R., Vaughan, T.G., Barido-Sottani, J., Duchêne, S., Fourment, M., Gavryushkina, A., et al. (2019). BEAST 2.5: An advanced software platform for Bayesian evolutionary analysis. PLoS Comput. Biol., 15, 1-28.

Broennimann, O., Fitzpatrick, M.C., Pearman, P.B., Petitpierre, B., Pellissier, L., Yoccoz, N.G., et al. (2012). Measuring ecological niche overlap from occurrence and spatial environmental data. Glob. Ecol. Biogeogr., 21, 481-497.

Jetz, W., Thomas, G.H., Joy, J.B., Hartmann, K. \& Mooers, A.O. (2012). The global diversity of birds in space and time. Nature, 491, 444-448. 


\section{A2: Projecting consequences of global warming for the functional diversity of fleshy-fruited plants and frugivorous birds along a tropical elevational gradient}

\section{Authors: Larissa Nowak, W. Daniel Kissling, Irene M.A. Bender, D. Matthias}

Dehling, Till Töpfer, Katrin Böhning-Gaese, Matthias Schleuning

Title: Projecting consequences of global warming for the functional diversity of fleshyfruited plants and frugivorous birds along a tropical elevational gradient

Status: published in Diversity and Distributions. 2019; 25:1362-1374.

https://doi.org/10.1111/ddi.12946

(open access article under the terms of the Creative Commons Attribution License)

\section{Author contributions:}

\section{(1) Concept and design}

LN, 80\%;

WDK, IMAB, DMD, MS, in total $20 \%$

\section{(2) Conducting tests and experiments}

n. a.

\section{(3) Compilation of data sets and figures}

DMD identified plant species in the field with the help from local experts, $100 \%$;

IMAB compiled plant elevational distributions from literature, $100 \%$;

DMD and IMAB measured plant morphological traits, in total $100 \%$;

DMD compiled bird elevational distributions from literature \& field observations, $100 \%$;

TT measured bird morphological data, 100\%;

IMAB derived local climate data from literature, 100\%;

LN compiled bird body mass data from literature, $100 \%$;

LN downloaded and processed bioclimatic data, 100\%;

LN derived lapse-rate data from literature and processed the data, 100\%;

LN prepared all data for analyses, $100 \%$;

LN prepared all figures and tables, $100 \%$

\section{(4) Analysis and interpretation of data}

LN computed the future projections of species' elevational ranges, $100 \%$;

LN analysed the functional diversity and functional correspondence of current and projected species' assemblages, 100\%;

LN interpreted the results, $90 \%$

with input from WDK, IMAB, DMD, TT, KBG, MS in total $10 \%$

\section{(5) Drafting of manuscript}

LN, 85\%;

WDK, IMAB, MS, in total $10 \%$;

DMD, TT, KBG, in total $5 \%$ 


\title{
Projecting consequences of global warming for the functional diversity of fleshy-fruited plants and frugivorous birds along a tropical elevational gradient
}

\author{
Larissa Nowak $^{1,2}$ (iD | W. Daniel Kissling ${ }^{3}$ | Irene M. A. Bender ${ }^{1,4,5}$ | \\ D. Matthias Dehling ${ }^{6}$ | Till Töpfer ${ }^{7}$ | Katrin Böhning-Gaese ${ }^{1,2}$ | Matthias Schleuning ${ }^{1}$ (D)
}

${ }^{1}$ Senckenberg Biodiversity and Climate Research Centre (SBiK-F), Frankfurt (Main). Germany

${ }^{2}$ Institute for Ecology. Evolution \& Diversity, Goethe University Frankfurt, Frankfurt (Main), Germany

${ }^{3}$ Institute for Biodiversity and Ecosystem Dynamics (IBED), University of Amsterdam, Amsterdam, The Netherlands

${ }^{4}$ German Centre for Integrative Biodiversit/ Research (iDiv) Halle-Jena-Leipzig, Leipzig, Germany

${ }^{5}$ Institute of Biology, Geobotany and Botanical Garden, Martin-Luther-University Halle-Wittenberg, Halle, Germany

${ }^{6}$ Centre for Integrative Ecology, School of Biological Sciences, University of Canterbury, Christchurch, New Zealand

${ }^{7}$ Zoological Research Museum Alexander Koenig (ZFMK), Bonn, Germany

\section{Correspondence}

Larissa Nowak, Senckenberg Biociversity and Climate Research Centre (SBiK-F)

60325 Frankfurt (Main), Germany. Email: larissa_nowak@web.de

Funding information

German Research Foundation, Grant/Award Number: DE 2754/1-1

Editor: Kenneth Feeley

\begin{abstract}
Aim: Species in ecological communities are linked by biotic interactions. It is therefore important to simultaneously study the impacts of global warming on interdependent taxa from different trophic levels. Here, we quantify current and potential future associations of functional diversity (based on multiple traits) and functional identity (based on individual traits) between interacting taxa using projection models under climate change.

Location: A tropical elevational gradient (500-3,500 $\mathrm{m}$ a.s.l.) in the Manú biosphere reserve, south-east Peru.

Methods: We investigated different scenarios of how species' elevational ranges might change under climate change based on projected future increases in mean annual temperature and current associations of species' elevational ranges with temperature. We computed the functional diversity and identity of current and potential future plant and bird communities based on morphological traits that have been shown to be important for plant-frugivore interactions. Finally, we tested for changes in the associations between projected functional diversity and identity of future plant and bird communities.

Results: Projected functional diversity of plants and birds decreased under range contraction and range shift scenarios at low elevations. At mid- and high elevations, functional diversity of both species groups increased most strongly under range expansion. Correspondence between plant and bird functional diversity was weakest under range contraction, while it remained strong under range expansion and shift. Similarly, the correspondence of projected plant and bird functional identity was weakest under a range contraction scenario.

Main conclusions: Our findings suggest that a scenario in which species are dispersal-limited and sensitive to increasing temperatures is likely to cause a functional mismatch between plant and bird communities along this tropical elevational gradient. This implies that certain functional types of plants could miss avian seed dispersers in
\end{abstract}

This is an open access article under the terms of the Creative Commons Attribution License, which permits use, distribution and reproduction in any medium, provided the original work is properly cited

(c) 2019 The Authors. Diversity and Distributions Published by John Wiley \& Sons Ltc

1362 wileyonlinelibrary.com/journal/ddi

Diversity and Distributions. 2019;25:1362-1374. 
the future. Our approach of studying functional diversity of interacting taxa could be more widely applied to identify potential future mismatches between trophic levels.

KEYWORDS

biotic interactions, climate change, ecological communities, functional correspondence, global change, seed dispersal, trait matching, trophic interactions

\section{1 | INTRODUCTION}

Current climate change happens rapidly and alters patterns of biodiversity (Bellard, Bertelsmeier, Leadley, Thuiller, \& Courchamp, 2012). In response to increasing temperatures, various plant and animal taxa have shifted their ranges poleward or upslope (Chen, Hill, Ohlemüller, Roy, \& Thomas, 2011; Feeley et al., 2011; Forero-Medina, Terborgh, Socolar, \& Pimm, 2011; Parmesan et al., 1999). Such range shifts might explain observed changes in the composition of ecological communities towards more warm-adapted species (Bowler \& Böhning-Gaese, 2017). Projecting changes in ecological communities is complex, as species differ in their vulnerability to climate change (Bowler, Heldbjerg, Fox, O'Hara, \& Böhning-Gaese, 2018; Voigt et al. 2003). For instance, species from different trophic levels have been reported to show distinct responses to global warming (Kharouba et al., 2018; Post \& Forchhammer, 2008). It is therefore important to investigate the effects of climate change on taxa from different trophic levels simultaneously (Schleuning et al., 2016).

Species in ecological communities are linked by biotic inter actions, such as trophic interactions between plants and animals (Bascompte \& Jordano, 2007). Independent species' range shifts could cause spatial mismatches between interacting species if the interdependent species shift their ranges differently due to differences in their thermal tolerances or dispersal abilities (Schweiger Settele, Kudrna, Klotz, \& Kühn, 2008). Future spatial mismatches between currently interacting species might trigger secondary range contractions and extinctions if lost interaction partners cannot be replaced (Schleuning et al., 2016; Schweiger et al., 2008). Such indirect, interaction-mediated consequences of climate change have often been overlooked and should be considered when studying the potential impacts of climate change on biodiversity (Blois, Zarnetske, Fitzpatrick, \& Finnegan, 2013).

According to the concept of ecological fitting (Janzen, 1985), trait matching between species determines who interacts with whom in ecological communities (Bender et al., 2018; Garibaldi et al., 2015). A widely documented example is the matching of bill length and corolla length in plant-hummingbird interactions (Maglianesi, Blüthgen, Böhning-Gaese, \& Schleuning, 2014; Weinstein \& Graham, 2017). In plant-frugivore interactions, bill width and fruit width are closely related (Dehling, Töpfer, et al., 2014; Moermond \& Denslow, 1985; Wheelwright, 1985). At the community level, the variation and dominant value of such functional traits can be estimated by metrics of the functional diversity of multiple traits and the functional identity of single traits (Díaz et al., 2007; Gagic et al., 2015). Both, functional diversity and identity, have been shown to correspond between communities of interacting species. For instance, community means of the mouth length of pollinators and the nectar accessibility of thei feeding plants improve predictions of plant reproduction (Garibaldi et al., 2015). Similarly, communities of fleshy-fruited plants and frugivorous birds show a high correspondence in their functional diversity and functional identity along elevational gradients (Dehling, Töpfer, et al., 2014; Vollstädt et al., 2017). However, potential future range shifts of species in response to climate change might alter the composition of ecological communities (Graham, Weinstein, Supp, \& Graham, 2017). Associated changes in the functional diversity and functional identity of ecological communities may trigger a disruption in the functional correspondence between interacting taxa. Therefore, integrative functional analyses are needed to identify ecological systems and regions that are prone to disruptions of functional correspondence between interacting taxa (Figure 1).

Mountains are global biodiversity hot spots (Antonelli et al., 2018; Jetz, Rahbek, \& Colwell, 2004) and are well suited to study functional associations between interacting taxa (Albrecht et al. 2018). Upslope range shifts of species in response to increasing temperatures might alter mountain biodiversity in distinct ways. The number of lowland species emigrating or going extinct from low elevations might exceed the number of persisting, warm-adapted species, resulting in a decline of diversity at low elevations (i.e., lowland biotic attrition; Colwell, Brehm, Cardelús, Gilman, \& Longino, 2008) Mid-elevations might experience elevated species turnover, due to immigrating lowland species and upslope shifts of species from midelevations (Corlett, 2011). At high elevations, diversity might accumulate as species from lower elevations immigrate to high elevations (Steinbauer et al., 2018), but high-elevation species might also face an increased extinction risk if their suitable environment contracts towards the mountaintop (Peters \& Darling, 1985). So far, there is empirical evidence for upslope shifts of the lower and/or the upper elevational range limits for a variety of plant and animal taxa across the globe (Freeman, Lee-Yaw, Sunday, \& Hargreaves, 2018; Freeman, Scholer, Ruiz-Gutierrez, \& Fitzpatrick, 2018). Consequences of elevational range shifts for biodiversity may be particularly severe in the tropics, where latitudinal temperature gradients are shallow and elevational range shifts more likely (Colwell et al., 2008). Tropical mountains are, therefore, particularly suitable for studying the effect of climate change on interacting species.

Here, we propose a new integrative analysis of functional diversity and projection models (Figure 1) to study potential future changes in the functional diversity of fleshy-fruited plant and frugivorous bird 


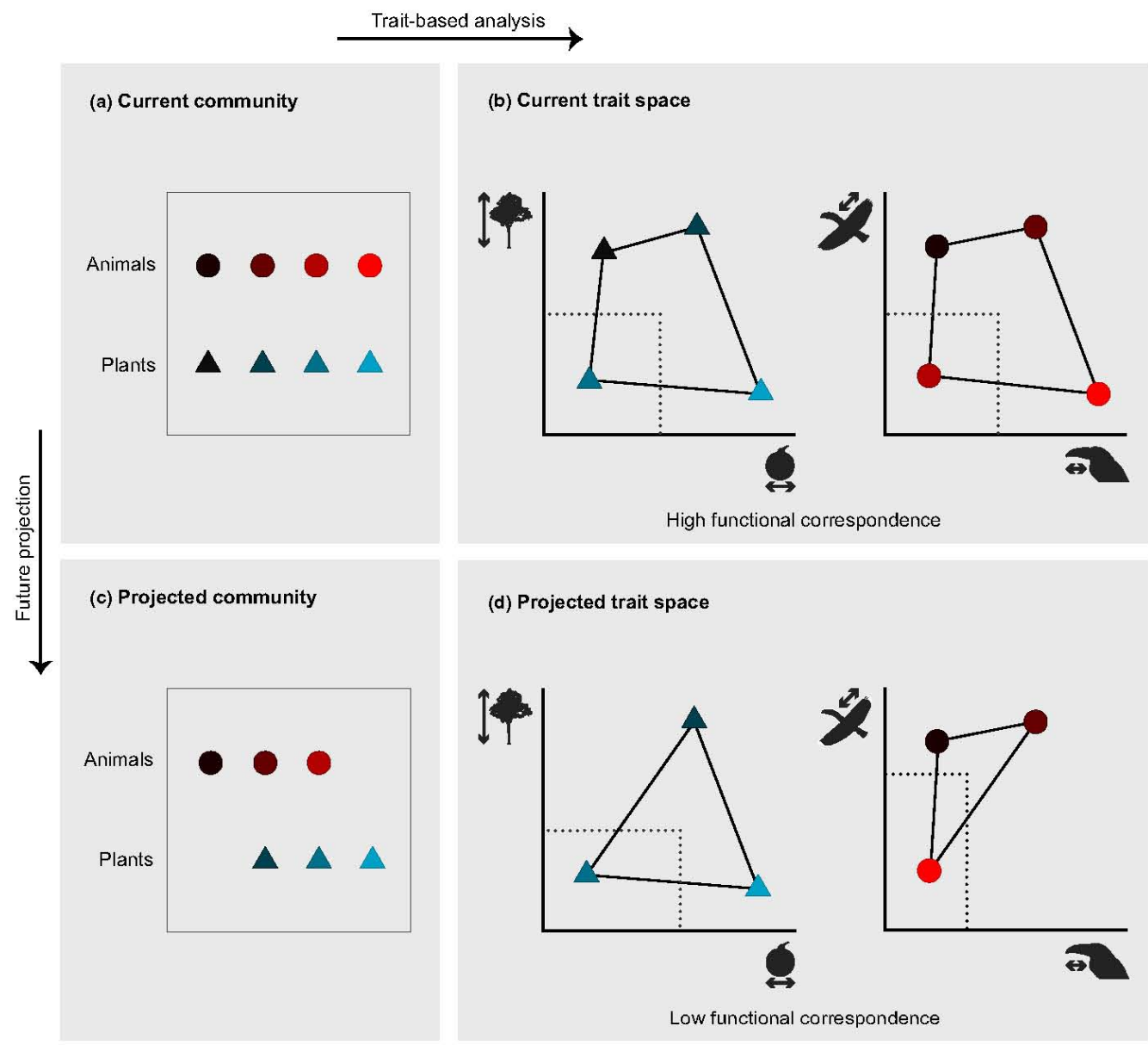

FIGURE 1 Projections of functional correspondence between interacting taxa from different trophic levels applying trait-based analyses. Shown are (a) hypothetical animal species (circles) and plant species (triangles) that co-occur in an ecological community. (b) Each species is defined by a set of functional traits that influence plant-animal interactions (e.g., fruit size and plant height and bill size and wing shape) Functional diversity is defined by the volume of the occupied multidimensional trait space (polygons in trait space) and the functional identity by community mean values of the respective traits (dashed lines to the individual axes). A high functional correspondence between plants and animals is indicated by similar values of functional diversity and identity. (c) A projection model, based on a future scenario, predicts different compositions of plant and animal communities. (d) Associated changes in functional diversity and identity of projected plant and animal communities could lead to a decrease in functional correspondence between plants and animals

communities along an elevational gradient in the tropical Andes of southeast Peru. We investigate multi-trait functional diversity and single-trait functional identity of plant communities based on fruit dimensions, plant height and crop mass and those of bird communities based on bill dimensions, wing pointedness and body mass. These species characteristics are relevant for trait matching in plant-frugivore networks (Bende et al., 2018; Dehling, Töpfer, et al., 2014; see further details in methods). We consider three previously reported scenarios of how species might alter their elevational ranges under increasing temperatures (Freeman,
Lee-Yaw, et al., 2018; Freeman, Scholer, et al., 2018; Figure 2a-c) and investigate changes in the projected functional correspondence of the two interacting species groups. We expect a decrease in functional diversity at low elevations under scenarios in which species shift their lower elevational range limit upslope (Colwell et al., 2008). Under scenarios in which species shift their upper elevational range limits upslope, we expect an increase in functional diversity at mid- and high elevations, because functionally more diverse species from low elevations might immigrate to mid- and high-elevation communities (Colwell 
Range contraction

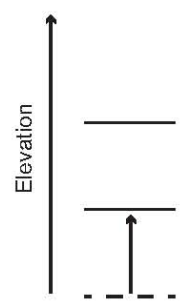

(a)

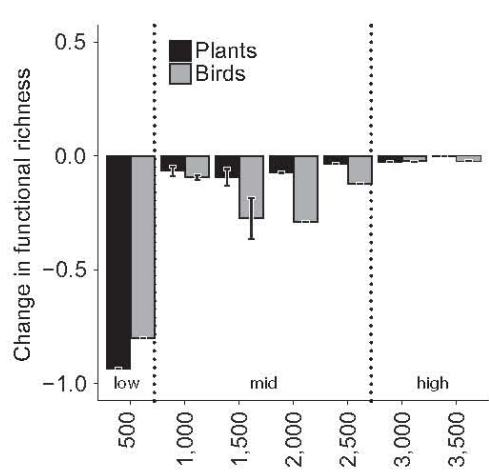

(d)

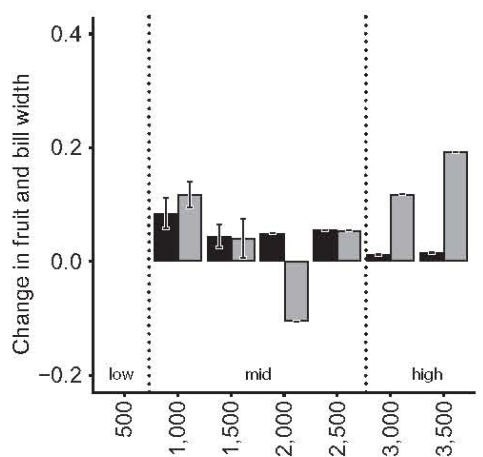

Range expansion

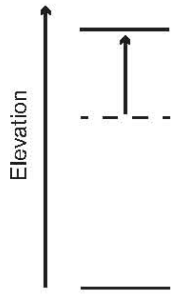

(b)
Range shift

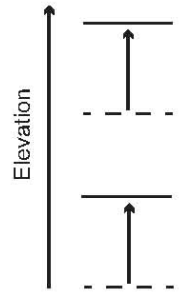

(c)

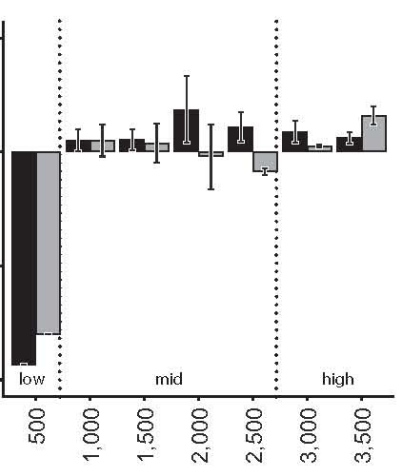

(f)

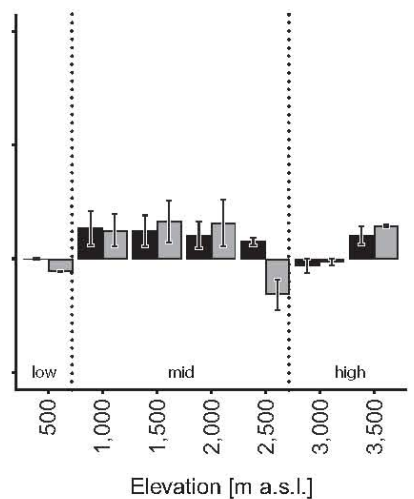

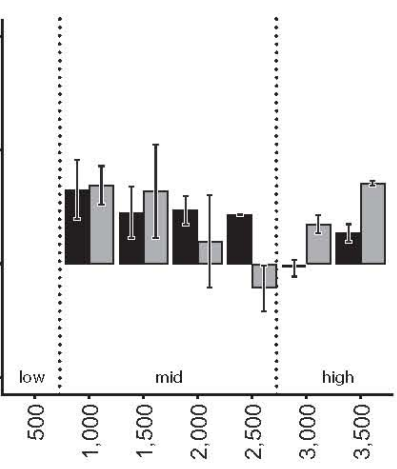

FIGURE 2 Projected changes in $(a-c)$ functional richness and $(d-f)$ mean fruit and bill width for fleshy-fruited plant (dark grey) and frugivorous bird (medium grey) communities under RCP 8.5 for the year 2080 . We applied three vertical dispersal scenarios for future projections of plant and bird communities along the Manú gradient, that is, range contraction, range expansion and range shift. Changes were computed as future functional richness minus current functional richness and future fruit and bill width minus current fruit and bill width, respectively. Given are mean values and standard deviation (error bars) over five general circulation models (CCSM4, HadGEM2-ES, MIROC 5, MRI-CGCM and NorESM). Dashed lines indicate low, mid- and high elevations. Functional identity of lowland communities could not be quantified under range contraction and shift scenarios because projected species richness was zero

et al., 2008; Dehling, Töpfer, et al., 2014). We expect particularly pronounced changes in functional diversity of bird communities at mid-elevations because species turnover between neighbouring frugivorous bird communities is high at these elevations along the Manú gradient (Dehling, Fritz, et al., 2014). This might lead to a decoupling of plant and bird functional diversity and identity under future scenarios. 


\section{METHODS}

\section{1 | Study system}

We studied communities of fleshy-fruited plants and frugivorous birds at seven elevations located every $500 \mathrm{~m}$ from 500 to $3,500 \mathrm{~m}$ a.s.I. in the Kosñipata valley in the Manú biosphere reserve in south-east Peru (hereafter "Manú gradient"). The entire study region ranges from approximately 250 to $3,750 \mathrm{~m}$ a.s.l. The forest along the Manú gradient comprises four main vegetation types (Patterson, Stotz, Solarit, Fitzpatrick, \& Pacheco, 1998). Lowland rainforest ( $\leq 500 \mathrm{~m}$ a.s.l.) is characterized by canopy trees up to $50 \mathrm{~m}$ high. Montane rainforest ( $500-1,500 \mathrm{~m}$ a.s. I.) lacks such tal trees and is characterized by denser understory vegetation. Cloud forest $(\sim 1,500-2,500 \mathrm{~m}$ a.s.l.) is intermittently covered in slowly rising clouds and typically hosts a high number of epiphytes and bryophyte layers on tree branches. Elfin forest ( $>2,500 \mathrm{~m}$ a.s.l.) consists of compact, densely branched trees and shrubs. Around the treeline $(-3,500 \mathrm{~m}$ a.s. I.), elfin forest turns into an irregula mosaic of forest and Puna grassland (Patterson et al., 1998). Al forest types are characterized by high precipitation, while temperature declines with increasing elevation from $24.3^{\circ} \mathrm{C}$ at $500 \mathrm{~m}$ a.s.l. to $7.3^{\circ} \mathrm{C}$ at $3,500 \mathrm{~m}$ a.s.l. (Girardin et al., 2010, 2013; Rapp \& Silman, 2012).

\subsection{Plant and bird communities}

To identify fleshy-fruited plant species co-occurring along the Manú gradient, we sampled an area of 1 ha (divided into 10 plots of $20 \mathrm{~m} \times 50 \mathrm{~m}$ ) every $500 \mathrm{~m}$ from 500 to $3,500 \mathrm{~m}$ a.s.. and recorded al plants with ripe fleshy fruits. Plants were identified as morphospecies, and samples of each species were taken. These samples were identified to species level in comparison with specimens from local herbaria. Each site was sampled once in the rainy season (Dece mbe to March) and once in the dry season (June to September) between December 2009 and September 2011. This resulted in representative samples of the trait diversity of the fleshy-fruited plant communities along the Manú gradient (Dehling, Töpfer, et al., 2014) although rarely fruiting species may not have been covered by ou sampling. Information on plant species' elevational distributions in Peru was taken from Brako and Zarucchi (1993), supplemented by data from Tropicos (2017; for about $3 \%$ of the analysed species). Because plant elevational ranges for entire Peru could overestimate local elevational ranges along the Manú gradient, we compared elevational range extents and functional diversity of current plant communities based on literature data and our own plot data (Figures \$1 and S2). Functional diversity of plants, based on the two dat sources, was positively correlated (Pearson $r=0.81, p=0.03, n=7$ elevations: Figure $\$ 2$ ). However, the plot data seemed to underestimate species' actual elevational range extents because about $80 \%$ of the analysed species were only recorded at a single elevation (Figure 51). Therefore, we decided to use the literature data that apparently provide a more comprehensive measure of the current elevational ranges of plants although this data source could slightly overestimate the current local ranges. We considered 392 plant species for which we had information on traits and elevational distribution in further analyses.

Information on co-occurring frugivorous bird species along the Manú gradient was compiled from local checklists (Merkord, 2010; Walker, Stotz, Pequeño, \& Fitzpatrick, 2006) supplemented by field observations (Dehling, Fritz, et al., 2014; Dehling, Sevillano, \& Morales, 2013). These occurrence data were compiled during repeated surveys along the Manú gradient over several years based on point counts, mist-netting or chance observations. If elevational ranges were derived from a country-wide guide book (Schulenberg, Stotz, Lane, O'Neill, \& Parker, 2010), estimates of functional diversity of current bird communities were very similar to those obtained through the Manú checklist (Pearson $r=0.98, p<0.001, n=7$ elevations; Figure 53). Because of its higher accuracy for the Manú gradient, we used range data from the Manú checklist. We considered all avian frugivore species that occur along the gradient, except ground-dwelling species (Tinamidae, Odontophoridae, Psophidae, Mitu). Ground-dwelling species have different fruit handling and foraging strategies than bird species that take fruit directly from the plant and their matching traits therefore differ compared to other guilds (Dehling, Töpfer, et al., 2014). Our final bird species pool included 217 frugivorous bird species.

We computed the current elevational range extent of each plant and bird species as the distance between its minimum and maximum elevational range limit (Tables A1 and A2 at DRYAD digital repository). Based on these data, we determined which plant and bird species occurred at each of the seven studied elevations $(500-3,500 \mathrm{~m}$ a.s.I.) and on the lowest ( $250 \mathrm{~m}$ a.s.l.) and the highest elevations (3,750 $\mathrm{m}$ a.s.l.) of the Manú gradient. An elevational resolution of $500 \mathrm{~m}$ was appropriate for describing elevational turnover across this wide elevational gradient, as it corresponds to the resolution of the available distribution data and has been applied in previous work comparing patterns in plant and bird diversity along this gradient (Dehling, Töpfer, et al., 2014).

\subsection{Plant and bird traits}

For all plant and bird species, we collected morphological traits that have been shown to determine interactions between plants and frugivores (Bender et al., 2018; Dehling, Töpfer, et al., 2014). Fruit width $(\mathrm{mm})$ and fruit length $(\mathrm{mm})$ of plants correspond to bill width $(\mathrm{mm})$ and bill length $(\mathrm{mm})$ of birds and relate to the size matching of bill and fruit (Dehling, Töpfer, et al., 2014; Moermond \& Denslow, 1985; Wheelwright, 1985). Fruit crop mass (i.e., the fresh mass (g) of a single fruit multiplied by the number of ripe fruits per plant) corresponds to avian body mass (g) and represents the matching between food resource availability and avian energy requirements, that is, larger frugivores tend to feed on plants that of fer a higher resource amount as this minimizes their foraging cost (Albrecht et al., 2018; Dehling. Töpfer, et al., 2014). Plant height ( $m$ ) corresponds to avian wing pointedness (estimated as Kipp's index: the distance from the tip of the first 
secondary to the tip of the longest primary feather [mm] divided by wing length $[\mathrm{mm}]$ ) and relates to trait matching of plant height at which fruits are offered and avian flight ability and manoeuvrability, that is, avian frugivores that forage in the canopy and fly long distances between fruiting plants have more pointed wings, while avian frugivores that forage in denser understory vegetation have more rounded wings which increases their manoeuvrability (Bender et al., 2018; Dehling, Töpfer, et al., 2014). Plant height and number of ripe fruits per tree were recorded in the field, while fruit dimensions and fresh fruit mass were measured on fruits collected during field surveys (on about 20 fruits per plant species). For 23 plant species ( $6 \%$ of the species pool), we used mean trait values at the genus level, as species-specific trait values were unavailable. Bill dimensions and wing pointedness were measured on museum specimens, following measurement protocols from Eck et al. (2011). Information on avian body mass was taken from the literature (Dunning, 2007). In our analyses, we used species means of all traits (see Tables A3 and A4 at DRYAD digital repository for trait values per plant and bird species and Appendix $\$ 1$ for details on the museum collection and identity of bird specimens).

\subsection{Functional diversity and identity}

We computed species richness, functional diversity and functional identity for each current and potential future plant and bird community. Species richness equals the number of species that occur in a community at a given elevation. Functional diversity was estimated as functional richness (Villéger, Mason, \& Mouillot, 2008). To reduce the weight of species with extreme traits, we log-transformed all traits, except Kipp's index, prior to the functional richness computation. Kipp's index was normally distributed and therefore needed no transformation. To compute functional richness, we calculated Euclidean distances between the species of a community based on differences in the respective traits (bill dimensions, pointedness of the wing and body mass for birds and fruit dimensions, plant height and crop size for plants) and used principle coordinate analysis to project species into a multidimensional trait space. We calculated functional richness as the inner volume of a minimum convex hull spanning all species of a community in trait space (Villéger et al., 2008). Species richness and functional richness were standardized to range between zero and one by dividing the value of the community at a specific elevation by the value of the entire species pool ( 392 plants species and 217 bird species, respectively, see Tables A5 and A6 at DRYAD digital repository for current and future functional diversity of plant and bird communities at each elevation). Standardized values are comparable among communities at different elevations and between plants and birds.

To estimate the functional identity of each current and future plant and bird community, we standardized plant and bird traits to zero mean and unit variance across the entire species pool of plants and birds, respectively. We then computed functional identity as the mean value of each trait across all species in a community, yielding directly comparable measures of functional identity for plant and bird communities (see Tables A7 and A8 at DRYAD digital repository for current and future functional identity of plant and bird communities at each elevation)

\subsection{Temperature projections, tropospheric lapse rate and vertical distances}

We calculated the projected temperature increase along the Manu gradient based on current and projected annual mean temperature downloaded from WorldClim (Hijmans, Cameron, Parra, Jones, \& Jarvis, 2005; details given in Appendix S2). We used projected data for the year 2080 (averaged over 2061-2080), according to five general circulation models (CCSM4, HadGEM2-ES, MIROC 5, MRICGCM and NorESM) and the representative concentration pathway (RCP) 8.5. This baseline scenario assumes continuing human population growth, little economic and technological developments, high energy demands and high greenhouse gas emissions (radiative forcing of $8.5 \mathrm{~W} / \mathrm{m}^{2}$ at the end of the century; Riahi et al., 2011). We chose this scenario because $\mathrm{CO}_{2}$ emissions have tracked the high end of emission scenarios until 2012 (Peters et al., 2012) and atmospheric $\mathrm{CO}_{2}$ concentration further continues to rise (Le Quéré et al., 2018).

We then used the tropospheric lapse rate (i.e., the rate at which temperature decreases with increasing elevation, Mokhov \& Akperov, 2006; details given in Appendix S2) to compute projected vertical distances (i.e., the distance a certain surface temperature will shift upslope under future conditions) for the Manú gradient ( $L a$ Sorte \& Jetz, 2010). We calculated the projected vertical distance for each elevation by dividing the projected anomaly in annual mean temperature of an elevation by the tropospheric lapse rate of that elevation (temperature anomaly $\left[{ }^{\circ} \mathrm{C}\right] /$ tropospheric lapse rate $\left[{ }^{\circ} \mathrm{C}\right.$ ) $\mathrm{km}]=$ vertical distance $[\mathrm{km}]$ )

\subsection{Projections of species' elevational ranges}

We approximated the current realized temperature niche of a species by its current elevational range and considered three scenarios of how the current elevational ranges of species could change under global warming (i.e., future temperature increases). We assumed that projected changes in species' elevational ranges are primarily driven by projected changes in temperature. Observed elevational range shifts of Andean species in response to recent (Feeley et al., 2011; Forero-Medina et al., 2011) and historic climate change (Hansen, Seltzer, \& Wright, 1994) support this assumption. At the Manú gradient, precipitation might be a less limiting factor for species' occurrences, as precipitation is currently high (Girardin et al., 2010, 2013 Rapp \& Silman, 2012) and projected to increase (Hijmans et al., 2005; Stocker et al., 2014). We further assumed that plant and bird species shift their elevational ranges only upslope because the Manú gradient is entirely covered by rainforest (Patterson et al., 1998).

We computed species-specific vertical distances as the mean vertical distance across all of the studied elevations at which a species currently occurs (La Sorte \& Jetz, 2010). The species-specific vertical distance approximates the distance a species would have to move upslope in order to track its current realized temperature niche under future global warming. In this computation, we considered vertical distances from nine grid cells at a resolution of $2.5 \mathrm{~min}$ that capture the entire elevational range of the Manú gradient 
(250-3,750 m a.s.l.; see Tables A1 and A2 at DRYAD digital repository for current elevational ranges and species-specific vertical distances of plant and bird species).

We implemented three vertical dispersal scenarios corresponding to observed changes in species' ranges on mountains (Freeman, Lee-Yaw, et al., 2018; Freeman, Scholer, et al., 2018). First, under the range contraction scenario (Figure 2a), we assumed that species cannot persist under temperatures that exceed their current realized temperature niche (i.e., species cannot tolerate or adapt to highe temperatures) and that species are unable to shift their ranges upslope. Therefore, species' lower elevational range limits shift upslope, while species' upper elevational range limits remain unchanged. Fo this scenario, we added the species-specific vertical distance to the lower limit of each species' current elevational range. Second, unde the range expansion scenario (Figure 2b), we assumed that species can tolerate or adapt to temperatures that exceed their current realized temperature niche, but also that species are able to shift thei elevational ranges upslope to track their current realized temperature niche. Therefore, species' lower elevational range limits remain unchanged, while species' upper elevational range limits shift upslope. For this scenario, we added the species-specific vertical distance to the upper limit of each species' current elevational range. Third, under the range shift scenario (Figure 2c), we assumed that species cannot persist under temperatures beyond their current realized temperature niche, but are able to shift their ranges upslope. Therefore, species' lower and upper elevational range limits shift upslope and species fully track their current realized temperature niche. For this scenario, we added the species-specific vertical distance to both the lower and the upper limits of each species ${ }^{t}$ current elevational range.

We applied the vertical dispersal scenarios to all plant and bird species, which resulted in one potential plant and one potential bird community at each elevation for each vertical dispersal scenario and for each general circulation model. We compared projected future functional diversity and functional identity values of plant and bird communities only between corresponding dispersal scenarios because we have no prior knowledge on how dispersal scenarios may differ between plants and birds. We also did not vary dispersal scenarios among individual species within the two groups as this would require a quantification of the dispersal ability of each individual species. Our projections do not aim to mimic reality, but to provide insights into how plant and bird communities and their functiona relationships could differ under different hypothetical scenarios.

\subsection{Statistical analyses}

We quantified projected changes in plant and bird functional diversity by subtracting the current functional diversity of a community from its projected functional diversity. We compared these projections to analogous projections of plant and bird species richness. Furthermore, we computed projected changes in the functional identity of plant and bird communities by subtracting the current mean trait value of a community from its projected mean trait value. Projected changes were computed separately for each global circulation model.
To test for a correspondence between the functional diversity and functional identity of plant and bird communities along elevation (Dehling, Topfer, et al., 2014), we fitted linear regression models with the functional diversity or functional identity of the plant communities as predictor and the functional diversity or functional identity of the corresponding bird communities as response. We did this for the current situation and for the three dispersal scenarios. These models were based on functional diversity and identity values that were averaged across the five general circulation models.

All computations were performed using $\mathrm{R}$ version 3.3.2 (R Core Team, 2016) and the packages "FD" (Laliberté \& Legendre, 2010; Laliberté, Legendre, \& Shipley, 2014) and "raster" (Hijmans, 2016).

\section{3 | RESULTS}

\subsection{Current patterns of functional diversity and identity}

Current plant species richness ranged from 276 (see Table A5 at DRYAD digital repository for species richness and functional diversity of plant communities at each elevation) at low elevations ( $500 \mathrm{~m}$ a.s.l.) to 76 species around the treeline $(3,500 \mathrm{~m}$ a.s.l.). The current bird species richness decreased from 126 species (see Table A6 at DRYAD digital repository) at low elevations to 19 species around the treeline. The current functional richness of plants (standardized relative to the full species pool) declined from 0.93 at low elevations to 0.09 around the treeline. The current standardized functional richness of bird species varied between 0.8 at low elevations and 0.12 around the treeline. Functional richness (Figure $3 a$ ) and species richness (Figure S6a) of current plant and bird communities were significantly positively related. Similarly, fruit width and bill width of current plant and bird communities were positively associated (Figure 4a). The other matching traits of plants and birds (mean fruit and bill length, plant height and Kipp's index, crop and body mass) showed largely similar patterns (Figures 57-59a).

\subsection{Projected elevational ranges and resulting changes in functional diversity}

Average projected increase in annual mean temperature across the Manú gradient varied between $3.0^{\circ} \mathrm{C}(S D= \pm 0.1, n=9$ elevations; MRI-CGCM) and $4.3^{\circ} \mathrm{C}(S D= \pm 0.1, n=9$ elevations; HadGEM2-ES) among the five global circulation models, with a mean temperature increase of $3.5 \pm 0.44^{\circ} \mathrm{C}$ across elevations and general circulation models. The average species-specific vertical distance that a species had to shift to stay within its current realized temperature niche until 2080 ranged between $486.6 \mathrm{~m}(S D= \pm 12.6 \mathrm{~m}, n=392$ plant species; MRI-CGCM) and $681.9 \mathrm{~m}(\mathrm{SD}= \pm 9.6 \mathrm{~m}$; HadGEM2ES) for plants. For birds, it varied from $483.8 \mathrm{~m}$ (SD $= \pm 13.1 \mathrm{~m}$, $n=217$ bird species; MRI-CGCM) to $679.8 \mathrm{~m}(S D= \pm 9.6 \mathrm{~m}$; HadGEM2-ES).

Plant and bird functional diversity changed under all vertical dispersal scenarios. At low elevations, functional richness 
FIGURE 3 Associations between plant and bird functional richness under (a) current conditions and under scenarios of (b) range contraction, (c) range expansion and (d) range shift. Dots indicate the mean value and error bars the standard deviation across five GCMs (CCSM4 HadGEM2-ES, MIROC 5, MRI-CGCM and NorESM). Horizontal error bars refer to plants, vertical error bars refer to birds. Given are slope estimates $(\beta)=$ standard errors and the $p$-values of linear models of bird functional richness against plant functional richness. Regression lines are represented as solid lines (for $p<0.05$ ) and dashed lines (for $0.05<p<0.1$ ). Low, mid- and high elevations are coloured in dark, medium and light grey, respectively
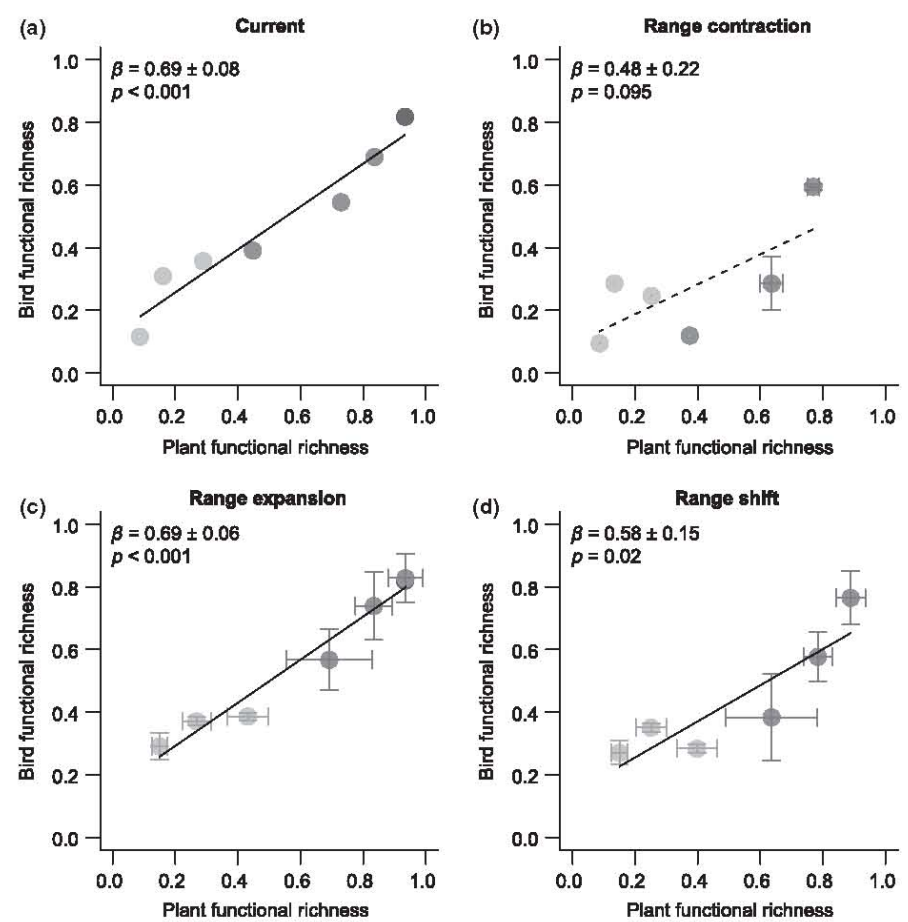

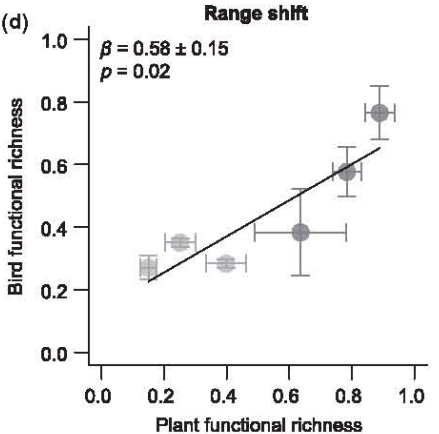

decreased strongly under range contraction and range shift scenarios (Figure 2a,c). At mid- and high elevations, functional richness increased under the range expansion scenario and, to a much lesser extent, under the range shift scenario. Increases were mostly weaker at high than at mid-elevations (Figure $2 b, c$ ). Projected changes in plant and bird species richness were overall similar to changes in functional richness, but were generally weaker (Figure 54). Mean fruit and bill width mostly increased at mid- and high elevations independent of the vertical dispersal scenarios (Figure $2 d-f$ ). Similarly, the mean values of fruit and bill length, plant height, wing pointedness, plant crop mass and avian body mass mostly increased under the different dispersal scenarios (Figure S5).

Projected future plant and bird functional richness corresponded least under the range contraction scenario (Figure $3 \mathrm{~b}$ ). This discrepancy between plant and bird functional richness resulted mostly from a stronger decrease in projected bird than plant functional richness at mid-elevations (Figure 2a). In contrast, under the range expansion and the range shift scenario, projected plant and bird functional richness was significantly positively associated (Figure $3 c$,d). Projected plant and bird species richness was significantly positively associated under each dispersal scenario (Figure S6).

In line with the findings for functional richness, the correspondence of projected plant and bird functional identity was weakest under a range contraction (Figure $4 \mathrm{~b}$ ) and highest under a range expansion scenario (Figure 4c). In addition, the correspondence of projected plant and bird functional identity was also weak under a range shift scenario (Figure 4d, Figures 57-59).

\section{DISCUSSION}

Our study simulated how different scenarios of temperature-driven range changes may influence the functional diversity of fleshyfruited plants and frugivorous birds along a tropical elevational gradient. Under a scenario in which species' elevational ranges contract in response to increasing temperatures, the projected correspondence of functional diversity and functional identity between plant and bird communities was lowest, while under range expansion functional associations persisted. Our results show how integrative analyses of functional diversity and projection models can be a powerful tool to explore scenarios under which disruptions of functional relationships between interacting species are most likely.

\section{1 | Projected changes in plant and bird} functional richness

At low elevations, plant and bird functional diversity were projected to decrease under range contraction and range shift scenarios. If temperature increases by about $3-4^{\circ} \mathrm{C}$, all fleshy-fruited plant and frugivorous bird species were projected to be lost from low elevations. 
(a)

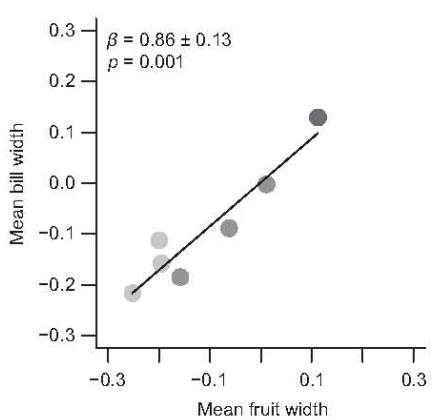

(c)

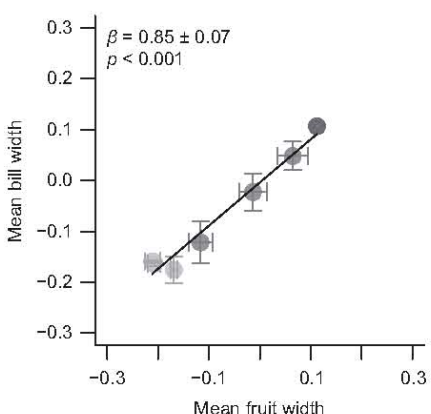

(b)

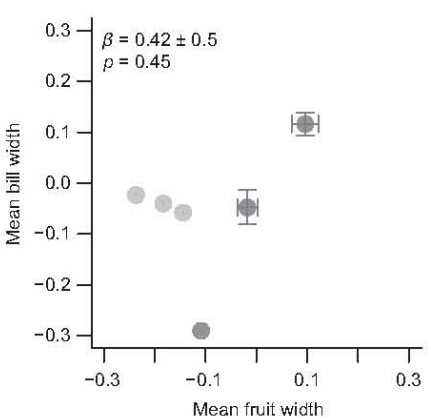

(d)

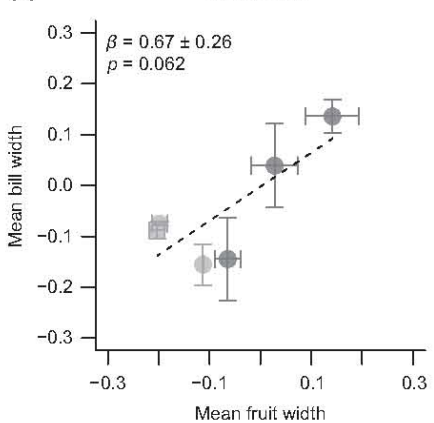

FIGURE 4 Associations between mean fruit width and mean bill width under (a) current conditions and under scenarios of (b) range contraction, (c) range expansion and (d) range shift. Dots indicate the mean value and error bars the standard deviation across five GCMs (CCSM4 HadGEM2-ES, MIROC 5, MRI-CGCM and NorESM). Horizontal error bars refer to plants, and vertical error bars refer to birds. Regression lines are represented as solid lines (for $p<0.05$ ) and dashed lines (for $0.05<p<0.1$ ). Low, mid- and high elevations are coloured in dark, medium and light grey, respectively
Such a projected decline is in line with the hypothesis of lowland biotic attrition (Colwell et al., 2008). However, lowland biotic attrition might be mitigated by species from warmer regions that immigrate to lowland communities (Anderson et al., 2012). This requires the existence of such warm-adapted species (Colwell et al., 2008) and their ability to disperse to the respective regions (Anderson et al., 2012) The strength of lowland biotic attrition will further depend on species' thermal tolerances (Feeley \& Silman, 2010). Occurrence-derived thermal tolerances of tropical lowland species might underestimate thermal tolerances as species possibly tolerate higher temperatures than currently realized (Feeley \& Silman, 2010). A global meta-analysis on past distributional changes in vascular plants and endo- and ectothermic animals indeed shows that most lowland species rather extend their ranges upslope than emigrating away from low elevations (Freeman, Lee-Yaw, et al., 2018). Furthermore, changes in the composition of lowland plant communities in France lag behind observed temperature increases between 1965 and 2008 (Bertrand et al., 2011). Nevertheless, a decrease in plant species richness was recently reported at low elevations in the Alps (Scherrer, Massy, Meier Vittoz, \& Guisan, 2017), suggesting that lowland biotic attrition is happening in some mountain areas.

At mid-elevations, functional richness increased under range expansion and, to a lesser extent, under range shift, indicating that species with functionally more extreme trait combinations were projected to immigrate to higher elevations. The current decline in functional richness with elevation suggests a filtering of species with extreme trait values, for example, large-billed and large-fruited species, towards high elevations (Dehling, Fritz, et al., 2014; Dehling, Töpfer, et al., 2014). Our projections of upslope dispersal suggest that communities at high elevations might become functionally more diverse in the future. Empirical studies indeed report increasing plant species richness at mid- and high elevations under climate change in North America (Savage \& Vellend, 2015; Sproull, Quigley, Sher, \& González, 2015) and Europe (Steinbauer et al., 2018). If global warming continues, a likely scenario would be a gradual increase in functional diversity of plant and bird communities at mid- and high elevations of the Manú gradient and on other tropical mountains.

\subsection{Potential future differences between plant and bird functional diversity and identity}

Projected plant and bird functional richness and identity corresponded least under the range contraction scenario. Low functional correspondence resulted from a stronger projected decline in bird than plant functional richness at mid-elevations and from a mismatch in mean trait values between plants and birds at mid- and high elevations. The high taxonomic and functional turnover between neighbouring bird communities at mid-elevations (Dehling, Fritz, et al., 
2014; Jankowski et al., 2013) might explain the strong effect of range contraction on these bird communities. Accordingly, many montane bird species are projected to be threatened if they are vulnerable to increasing temperatures and limited in their vertical dispersal ( $L a$ Sorte \& Jetz, 2010). In current plant-frugivore communities across the Andes, certain functional types of frugivorous birds interact with certain functional types of fleshy-fruited plants (Bender et al., 2018) For instance, large-beaked bird species mostly feed on large fruits, species with pointed wings tend to forage in the canopy and species with a large body mass prefer plants with a large crop mass (Dehling, Töpfer, et al., 2014; Wheelwright, 1985). A higher plant than bird functional richness, as found at mid-elevations under the range contraction scenario, suggests that certain functional types of plants might lack seed dispersers with matching functional traits in future communities (Bender et al., 2018; Dehling, Töpfer, et al., 2014). This is in line with the projected mismatch between fruit and bill width at $2,000 \mathrm{~m}$, where mean bill width was projected to decrease in the future. At this elevation, the dispersal of particular plant species might decrease because the loss of large-bodied frugivores reduces, particularly, the dispersal of large-fruited plant species (Markl et al., 2012). The projected increase in avian bill width at high elevations might have less severe consequences as broad-billed bird species tend to be flexible in their fruit choice (Bender et al., 2017; Moermond \& Denslow, 1985; Wheelwright, 1985). Generally, avian flexibility to switch to other fruit resources could mitigate the risk to lose seed-dispersal functions under climate change. In the long run, however, a mismatch between plant and bird traits would likely trigger evolutionary changes in plant communities, for example, at the expense of large-fruited plant species (Onstein et al., 2018).

Under range expansion, projected differences between plant and bird functional richness and identity were smallest. This sug gests that losing seed-dispersal functions under climate change is less likely if species are able to shift their upper elevational range limits upslope. Accordingly, the projected range loss of montane bird species under climate change was least severe if species were able to disperse vertically (La Sorte \& Jetz, 2010). Indeed, many species across the globe already shifted their upper elevational range limits upslope (Freeman, Lee-Yaw, et al., 2018; Freeman, Scholer, et al., 2018). Projected changes were less conclusive under a range shift scenario. Associations between functional richness and, in particular, individual functional traits were projected to decrease under range shift. However, variation in projections was also highest under this scenario, indicating that future correspondence between plant and bird communities was most difficult to project if both lower and upper elevational ranges of species were assumed to change.

\subsection{Integrative analysis of functional diversity under climate change}

Our approach provides a new way to integrate species ${ }^{t}$ occurrences, functional traits and projection models to assess consequences of global warming for the functional correspondence between interacting taxa (Figure 1). The proposed approach can yield new information on how functional associations between taxa are likely to change under future climate change and is useful to identify under which scenarios functional mismatches between taxa are most likely to happen. Such analyses could be applied to a wide range of other taxa that are linked by trophic interactions and only require occurrence data for both taxa as well as information on the functional traits that mediate their interactions, such as traits that determine the probability of plant-pollinator or predator-prey interactions (Webb \& Shine, 1993; Weinstein \& Graham, 2017). Such trait-based approaches are timely as more and better trait data are increasingly becoming available (Kissling et al., 2018). Ultimately, a formal integration of such trait-based approaches into projection models is desirable and could improve how biotic interactions are accounted for in species distribution models (Dormann et al., 2018).

Our study is a first step towards comparative analyses of interacting taxa under climate change. We particularly encourage future studies that use more fine-scale occurrence data than those that are available for our study system. Moreover, the future projection models we apply rely on many assumptions and a better integration of species-specific responses in terms of physiological thermal tolerances (Khaliq, Hof, Prinzinger, Böhning-Gaese, \& Pfenninger, 2014 Londoño, Chappell, Jankowski, \& Robinson, 2017) and different dispersal capacities of species (Grewe, Hof, Dehling, Brandl, \& Brändle, 2013) could improve model projections. A promising way forward could also be the use of time-series data that document how occurrences of interacting taxa have changed over time (Burkle, Marlin \& Knight, 2013), in order to quantify temporal variation in the functional associations between taxa.

\section{CONCLUSIONS}

To our knowledge, this is the first study that simultaneously investigates how projected range change might influence the functional diversity of interacting taxa under climate change. Our results suggest that under a scenario in which species are sensitive to increasing temperatures and dispersal-limited, the potential for future functional mismatches between fleshy-fruited plants and frugivorous birds is highest. For conserving tropical mountain biodiversity, it might therefore be important to enable species to shift their ranges upslope and to plan for movement corridors along elevational gradients (Moore, Robinson, Lovette, \& Robinson, 2008). Our approach of integrating functional diversity analyses and projection models can be widely applied to a range of interacting taxa linked by trophic interactions and could help to identify future scenarios under which biotic interactions between taxa are most vulnerable.

\section{ACKNOWLEDGEMENTS}

R. van den Elzen (ZFMK Bonn), R. Prŷs-Jones and M. P. Adams (NHM Tring), G. Mayr (SMF Frankfurt) and R. Winkler (NMB Basel) provided access to bird collections. M. Hennen, J. Bates and D. Willard (FMNH 
Chicago) sent specimens, and J. V. Remsen and S. W. Cardiff (LSUZM Baton Rouge) and D. Willard (FMNH Chicago) provided additional measurements. We thank R. Diesener, S. Frahnert, C. Bracker, P.-R. Becker, J. Fjeldså, N. Krabbe and J. Mlíkovsky for information about collection holdings. We are grateful to Jörg Albrecht, Susanne Fritz, Christian Hof, Thomas Müller, Eike-Lena Neuschulz, Marjorie Sorensen and Diana Bowler for valuable comments on the research ideas and the development of the modelling approach. W.D.K. acknowledges a University of Amsterdam starting grant. D.M.D. was supported by a grant from the German Research Foundation (DE 2754/1-1). Field work at Manu was conducted under the permits 041-2010-AG-DGFFSDGEFFS, 008-2011-AG-DGFFS-DGEFFS, 01-C/C-2010SERNANP-JPNM, and 01-2011-SERNANP-PNM-JEF. Finally, we want to thank Kenneth Feeley and four anonymous reviewers for giving us valuable advice to improve the manuscript.

\section{DATA ACCESSIBILITY}

Species' current elevational ranges and species-specific vertical distances (Tables A1 and A2), morphological trait data of plants and birds (Tables $\mathrm{A} 3$ and $\mathrm{A} 4$ ) as well as current and projected species richness, functional richness (Tables $A 5$ and $A 6$ ) and functional identity (Tables A7 and A8) of plant and bird communities are provided at the DRYAD data repository (https://doi.org/10.5061/dryad.c0n737b).

\section{ORCID}

Larissa Nowak (D) https://orcid.org/0000-0002-1910-8041 Matthias Schleuning https://orcid.org/0000-0001-9426-045X

\section{REFERENCES}

Albrecht, J., Classen, A., Vollstädt, M. G. R., Mayr, A., Mollel, N. P. Schellenberger Costa, D., ... Schleuning, M. (2018). Plant and animal functional diversity drive mutualistic network assembly across an elevational gradient. Nature Communications, 9, 3177. https://doi. org/10.1038/s41467-018-05610-w

Anderson, A. S., Reside, A. E., Vanderwal, J. J., Shoo, L. P., Pearson, R. G., \& Williams, S. E. (2012). Immigrantsandrefugees:The importance of dispersal in mediating biotic attrition under climate change. Global Change Biology, 18. 2126-2134. https://doi.org/10.1111/j.1365-2486.2012.02683.x

Antonelli, A., Kissling, W. D., Flantua, S. G. A., Bermúdez, M. A., Mulch, A., Muellner-Riehl, A. N., ... Hoorn, C. (2018). Geological and climatic influences on mountain biodiversity. Nature Geoscience, 11, 718-725. https://doi.org/10.1038/s41561-018-0236-z

Bascompte, J, \& Jordano, P. (2007) Plant-animal mutualistic networks: The architecture of biodiversity. Annual Review of Ecology, Evolution, and Systematics, 38, 567-593. https://doi.org/10.1146/annurev.ecols ys.38.091206.095818

Bellard, C., Bertelsmeier, C., Leadley, P., Thuiller,W., \& Courchamp,F. (2012) Impacts of climate change on the future of biodiversity. Ecology Letters, 15, 365-377. https://doi.org/10.1111/j.1461-0248.2011.01736.x

Bender, I. M. A., Kissling, W. D., Blendinger, P. G., Böhning-Gaese, K. Hensen, I., Kühn, I., ... Schleuning, M. (2018). Morphological trait matching shapes plant-frugivore networks across the Andes. Ecography, 41, 1-10. https://doi.org/10.1111/ecog.03396
Bender, I. M. A., Kissling, W. D., Böhning-Gaese, K., Hensen, I., Kühn, I., Wiegand, T., ... Schleuning, M. (2017). Functionally specialised birds respond flexibly to seasonal changes in fruitavailability. Journal of Animal Ecology, 86, 800-811. https://doi.org/10.1111/1365-2656.12683

Bertrand, R., Lenoir, J., Piedallu, C., Riofrío-Dillon, G., de Ruffray, P., Vidal, C., ... Gégout, J.-C. (2011). Changes in plant community composition lag behind climate warming in lowland forests. Nature, 479, 517-520. https://doi.org/10.1038/nature10548

Blois, J. L., Zarnetske, P. L., Fitzpatrick, M. C. \& Finnegan, S. (2013). Climate change and the past, present, and future of biotic interactions. Science, 341, 499-504. https://doiorg/10.1126/science.1237184

Bowler, D, \& Böhning-Gaese, K. (2017). Improving the communitytemperature index as a climate change indicator. PLOS ONE, 12, e0184275. https://doi.org/10.1371/journal.pone.0184275

Bowler, D., Heldbjerg, H., Fox, A. D., O'Hara, R. B., \& Böhning-Gaese, K. (2018). Disentangling the effects of multiple environmental drivers on population changes within communities. Journal of Animal Ecology, 87, 1034-1045. https://doi.org/10.1111/1365-2656.12829

Brako, L., \&. Zarucchi, J. L. (1993). Catalogue of the flowering plants and Gymnosperms of Peru. Catálogo de las Angiospermas y Gimnospermas del Perú. Monographs in systematic botany from the Missouri Botanical Garden 45.

Burkle, L. A., Marlin, J. C., \& Knight, T. M. (2013). Plant-Pollinator interactions over 120 years: Loss of species, co-occurrence, and function. Science, 339(6127), 1611-1615. https://doi.org/10.1126/scien ce. 1232728

Chen, I. C., Hill, J. K., Ohlemüller, R., Roy, D. B., \& Thomas, C. D. (2011) Rapid range shifts of species associated with high levels of climate warming. Science, 333, 1024-1026. https://doi.org/10.1126/scien ce. 1206432

Colwell, R. K., Brehm, G., Cardelús, C. L., Gilman, A. C., \& Longino, J. T. (2008). Global warming, elevational range shifts, and lowland biotic attrition in the wet tropics. Science, 322, 258-261. https://doi. org/10.1126/science.1162547

Corlett, R. T. (2011). Impacts of warming on tropical lowland rain forests. Trends in Ecology and Evolution, 26, 606-613. https://doi. org/10.1016/i.tree.2011.06.015

Dehling, D. M., Fritz, S. A., Töpfer, T., Päckert, M., Estler, P., BöhningGaese, K., \& Schleuning, M. (2014). Functional and phylogenetic diversity and assemblage structure of frugivorous birds along an elevational gradient in the tropical Andes. Ecography, 37, 1047-1055. https://doi.org/10.1111/ecog.00623

Dehling, D. M., Sevillano, C. S., \& Morales, L. V. (2013). Upper and lower elevational extremes of Andean birds from south-east Peru. Boletín informativo, 8, 32-38.

Dehling, D. M., Töpfer, T., Schaefer, H. M., Jordano, P., Böhning-Gaese, K., \& Schleuning, M. (2014). Functional relationships beyond species richness patterns: Trait matching in plant-bird mutualisms across scales. Global Ecology and Biogeography, 23, 1085-1093. https://doi. org/10.1111/geb.12193

Díaz, S., Lavorel, S., de Bello, F., Quétier, F., Grigulis, K., \& Robson, T. M. (2007). Incorporating plant functional diversity effects in ecosystem service assessments. Proceedings of the National Academy of Sciences of the United States of America, 104, 20684-20689. https:// doi.org/10.1073/pnas.0704716104

Dormann, C. F., Bobrowski, M., Dehling, D. M., Harris, D. J., Hartig, F., Lischke, H., ... Kraan, C. (2018). Biotic interactions in species distribution modelling: 10 questions to guide interpretation and avoid false conclusions. Global Ecology and Biogeography, 27, 1004-1016. https://doi.org/10.1111/geb.12759

Dunning, J. B. (2007). CRC handbook of avian body masses. Boca Raton, FL: CRC Press.

Eck, S., Töpfer, T., Fiebig, J., Heynen, I., Fiedler, W., Nicolai, B.,... Woog, F. (2011). Measuring birds. Minden, Germany: Christ Media Natur. 
Feeley, K. J., \& Silman, M. R. (2010). Biotic attrition from tropical forest correcting for truncated temperature niches. Global Change Biology, 16, 1830-1836. https://doi.org/10.1111/j.1365-2486.2009.02085.x

Feeley, K J. Silman, M. R. Bush, M. B. Farfan, W Cabrera, K. G. Malhi, Y., ... Saatchi, S. (2011). Upslope migration of Andean trees. Journal of Biogeography, 38, 783-791. https://doi.org/10.1111/ j.1365-2699.2010.02444.x

Forero-Medina, G., Terborgh, J., Socolar, S. J., \& Pimm, S. L. (2011) Elevational ranges of birds on a tropical montane gradient lag behind warming temperatures. PLOS ONE, 6, e28535. https://doi. org/10.1371/journal.pone.0028535

Freeman, B. G., Lee-Yaw, J. A., Sunday, J. M., \& Hargreaves, A. L. (2018) Expanding, shifting and shrinking: The impact of global warming on species' elevational distributions. Global Ecology and Biogeography, 27, 1268-1276. https://doi.org/10.1111/geb.12774

Freeman, B. G., Scholer, M. N., Ruiz-Gutierrez, V., \& Fitzpatrick, J. W. (2010). Climate change causes upslope shifts and mountaintop ex tirpations in a tropical bird community. Proceedings of the Nationa Academy of Sciences of the United States of America, 115, 1198211987. https://doi.org/10.1073/pnas.1804224115

Gagic, V., Bartomeus, I., Jonsson, T., Taylor, A., Winqvist, C., Fischer, C. ... Bommarco, R. (2015). Functional identity and diversity of animals predict ecosystem functioning better than species-based indices. Proceedings of the Royal Society B: Biological Sciences, 282, 20142620 https://doi.org/10.1098/rspb.2014.2620

Garibaldi, L. A., Bartomeus, I., Bommarco, R., Klein, A. M., Cunningham, S. A., Aizen, M. A., ... Woyciechowski, M. (2015). Trait matching of flower visitors and crops predicts fruit set better than trait diversity. Journal of Applied Ecology, 52, 1436-1444. https://doi org/10.1111/1365-2664.12530

Girardin, C. A. J., Aragão, L. E. O. C.. Malhi, Y., Huaraca Huasco, W. Metcalfe, D. B., Durand, L., ... Whittaker, R. J. (2013). Fine root dynamics along an elevational gradient in tropical Amazonian and Andean forests. Global Biogeochemical Cycles, 27, 252-264. https:/ doi.org/10.1029/2011gb004082

Girardin, C. A. J, Malhi, Y. Aragao, L, Mamani, M., Huaraca Huasco, W. \& Durand, L. (2010). Net primary productivity allocation and cyclins of carbon along a tropical forest elevational transect in the Peruvian Andes. Global Change Biology, 16,3176-3192. https://doi.org/10.104 3/0003-9985(2002)126<0464:GEPH>2.0.CO;2

Graham, L. J., Weinstein, B. G., Supp, S. R., \& Graham, C. H. (2017). Future geographic patterns of novel and disappearing assemblages across three dimensions of diversity: A case study with Ecuadorian hummingbirds. Diversity and Distributions, 23, 944-954. https://doi. org/10.1111/ddi.12587

Grewe, Y., Hof, C., Dehling, D. M., Brandl, R., \& Brändle, M. (2013). Recent range shifts of European dragonflies provide support for an inverse relationship between habitat predictability and dispersal. Globa Ecology and Biogeography, 22, 403-409. https://doi.org/10.1111/ geb.12004

Hansen, B. C. S., Seltzer, G. O., \& Wright, H. E. Jr (1994). Late Quaternary vegetational change in the central Peruvian Andes. Palaeogeography, Palaeoclimatology, Palaeoecology, 109, 263-285. https://doi. org/10.1016/0031-0182(94)90179-1

Hiimans, R. J. (2016). Raster: geographic data analysis and modeling. R package version $2.5-8$

Hijmans, R. J., Cameron, S. E., Parra, J. L., Jones, P. G., \& Jarvis, A. (2005) Very high resolution interpolated climate surfaces for $g$ lobal land areas. International Journal of Climatology, 25, 1965-1978. https:// doi.org/10.1002/joc.1276

Jankowski, J. E., Merkord, C. L., Rios, W. F., Cabrera, K. G., Revilla, N. S., \& Silman, M. R. (2013). The relationship of tropical bird communities to tree species composition and vegetation structure along an Andean elevational gradient. Journal of Biogeography, 40, 950-962. https:// doi.org/10.1111/ibi.12041
Janzen, D. H. (1985). On ecological fitting. Oikos, 45, 308-310. https:// doi.org/10.2307/3565565

Jetz. W., Rahbek, C., \& Colwell, R. K. (2004). The coincidence of rarity and richness and the potential signature of history in centres of endemism. Ecology Letters, 7 1180-1191. https://doi. org/10.1111/j.1461-0248.2004.00678.x

Khaliq, I., Hof, C., Prinzinger, R., Böhning-Gaese, K., \& Pfenninger, M. (2014). Global variation in thermal tolerances and vulnerability of endotherms to climate change. Proceedings of the Royal Society B: Biological Sciences, 281, 20141097 https://doi.org/10.1098/ rspb.2014.1097

Kharouba, H. M., Johan, E., Gelman, A., Bolmgren, K., Allen, J. M., Travers, S. E., \& Wolkovich, E. M. (2018). Global shifts in the phenological synchrony of species interactions over recent decades. Proceedings of the National Academy of Sciences of the United States of America, 115, 5211-5216. https://doi.org/10.1073/pnas.1714511115

Kissling, W. D., Walls, R., Bowser, A., Jones, M. O., Kattge, J., Agosti, D., ... Guralnick, R. P. (2018). Towards global data products of Essentia Biodiversity Variables on species traits. Nature Ecology and Evolution, 2, 1531-1540. https://doi.org/10.1038/s41559-018-0667-3

La Sorte, F. A., \& Jetz, W. (2010). Projected range contractions of montane biodiversity under global warming. Proceedings of the Royal Society B: Biological Sciences, 277, 3401-3410. https://doi.org/10.1098/ rspb.2010.0612

Laliberté, E., \& Legendre, P. (2010). A distance-based framework for measuring functional diversity from multiple traits.pdf. Ecology, 91 299-305. https://doi.org/10.1890/08-2244.1

Laliberté, E., Legendre, P., \& Shipley, B. (2014). FD: measuring functional diversity from multiple traits, and other tools for functional ecology. $\mathrm{R}$ package version 1.0-12.

Le Quéré, C., Andrew, R. M., Friedlingstein, P., Sitch, S., Pongratz, J., Manning, A. C., ... Zhu, D.(2018). Global carbon budget 2017. Earth System Science Data Discussions, 10, 10, 405-448. https://doi. org/10.5194/essd-2017-123

Londoño, G. A., Chappell, M. A., Jankowski, J. E., \& Robinson, S. K. (2017). Do thermoregulatory costs limit altitude distributions of Andean forest birds? Functional Ecology, 31, 204-215. https://dol. org/10.1111/1365-2435.12697

Maglianesi, M. A., Blüthgen, N., Böhning-Gaese, K., \& Schleuning, M. 2014). Morphological traits determine specialization and resource use in plant-hummingbird networks in the neotropics. Ecology, 95, 3325-3334. https://doi.org/10.1890/13-2261.1

Markl, J. S., Schleuning, M., Forget, P. M., Jordano, P., Lambert, J. E., Traveset, A., ... Böhning-Gaese, K. (2012). Meta-analysis of the effects of human disturbance on seed dispersal by animals. Conservation Biology, 26, 1072-1081. https://doi,org/10,1111/j.

Merkord, C. L. (2010). Seasonality and elevational migration in an Andean bird community. University of Missouri-Columbia.

Moermond, T. C., \& Denslow, J. S. (1985). Neotropical avian frugivores: patterns of behavior, morphology, and nutrition, with consequences for fruit selection. Ornithological Monographs, 36, 865-897. https:// doi.org/10.2307/40168322

Mokhov, I. I., \& Akperov, M. G. (2006). Tropospheric lapse rate and its relation to surface temperature from reanalysis data. Izvestiya, Atmospheric and Oreanic Physics, 42, 430-438. https://doi.org/10.1134/50001 433806040037

Moore, R. P., Robinson, W. D., Lovette, I. J., \& Robinson, T. R. (2008) Experimental evidence for extreme dispersal limitation in tropical forest birds. Ecology Letters, 11, 960-968. https://doi. org/10.1111/j.1461-0248.2008.01196.x

Onstein, R. E., Baker, W. J., Couvreur, T. L. P., Faurby, S., Herrera-Alsina, L., Svenning, J.-C., \& Kissling, W. D. (2018). To adapt or go extinct? The fate of megafaunal palm fruits under past global change. Proceedings of the Royal Society B: Biological Sciences, 285, 20180882. https://doi. org/10.1098/rspb.2018.0882 
Parmesan, C., Ryrholm, N., Stefanescu, C., Hill, J. K., Thomas, C. D., Descimon, H., ... Warren, M. (1999). Poleward shifts in geographical ranges of butterfly species associated with regional warming. Nature, 399, 579-583. https://doi.org/10.1038/21181

Patterson, B. D., Stotz, D. F., Solarit, S., Fitzpatrick, J. W., \& Pacheco, V. (1998). Contrasting patterns of elevational zonation for birds and mammals in the Andes of southeastern Peru. Journal of Biogeography, 25, 593-607. https://doi.org/10.1046/j.1365-2699.1998.2530593.x

Peters, G. P., Andrew, R. M., Boden, T., Canadell, J. G., Ciais, P., Le Quéré, C, ... Wilson, C. (2012). The challenge to keep global warming below $2^{\circ} \mathrm{C}$. Nature Climate Change, 3, 4. https://doi.org/10.1038/nclimate1783

Peters, R. L., \& Darling, J. D. S. (1985). The greenhouse effect and nature reserves. BioScience, 35, 707-717. https://doi.org/10.2307/1310052

Post, E., \& Forchhammer, M. C. (2008). Climate change reduces reproductive success of an Arctic herbivore through trophic mismatch. Philosophical Transactions of the Royal Society B: Biological Sciences, 363, 2369-2375. https://doi.org/10.1098/rstb.2007.2207

$\mathrm{R}$ Core Team (2016). R: A language and environment for statistical computing. Vienna, Austria: R Foundation for Statistical Computing. Retrieved from https://www.r-project.org/

Rapp, J. M., \& Silman, M. R. (2012). Diurnal, seasonal, and altitudinal trends in microclimate across a tropical montane cloud forest. Climate Research, 55, 17-32. https://doiorg/10.3354/cr01127

Riahi, K., Rao, S., Krey, V., Cho, C., Chirkov, V., Fischer, G., ... Rafaj, P. (2011). RCP 8.5-A scenario of comparatively high greenhouse gas emissions. Climatic Change, 109, 33-57. https://doi.org/10.1007/ s10584-011-0149-y

Savage, J., \& Vellend, M. (2015). Elevational shifts, biotic homogenization and time lags in vegetation change during 40 years of climate warming. Ecography, 38, 546-555. https://doi.org/10.1111/ecog.01131

Scherrer, D., Massy, S., Meier, S., Vittoz, P., \& Guisan, A. (2017). Assessing and predicting shifts in mountain forest composition across 25 years of climate change. Diversity and Distributions, 23, 517-528. https:// doi.org/10.1111/ddi.12548

Schleuning, M., Fründ, J., Schweiger, O., Welk, E., Albrecht, J., Albrecht, M., ... Hof, C. (2016). Ecological networks are more sensitive to plant than to animal extinction under climate change. Nature Communications, 7, 13965. https://doi.org/10.1038/ncomms13965

Schulenberg, T. S., Stotz, D. F., Lane, D. F., O'Neill, J. P., \& Parker, T. A. III (2010). Birds of Peru: Revised and updated edition (Vol. 63). Princeton, $\mathrm{NJ}$ : Princeton University Press.

Schweiger, O., Settele, J., Kudrna, O., Klotz, S., \& Kühn, I. (2008). Climate change can cause spatial mismatch of trophically interacting species. Ecology, 89, 3472-3479. https://doi.org/10.1890/07-1748.1

Sproull, G. J., Quigley, M. F., Sher, A., \& González, E. (2015). Long-term changes in composition, diversity and distribution patterns in four herbaceous plant communities along an elevational gradient. Journo of Vegetation Science, 26, 552-563. https://doi.org/10.1111/jvs.12264

Steinbauer, M. J., Grytnes, J.-A., Jurasinski, G., Kulonen, A., Lenoir, J., Pauli, H., ... Wipf, S. (2018). Accelerated increase in plant species richness on mountain summits is linked to warming. Nature, 556 , 231-236. https://doi.org/10.1038/s41586-018-0005-6

Stocker, T. F., Qin, D., Plattner, G.-K., Tignor, M. M. B., Allen, S. K., Boschung, J., ... Midgley, P. M. (2014). In Intergovernmental Panel on Climate Change (Ed). Climate Change 2013 - The Physical Science Basis. Cambridge, UK: Cambridge University Press.

Tropicos (2017). Tropicos. Retrieved from http://www.tropicos.org

Villéger, S., Mason, N. W. H., \& Mouillot, D. (2008). New multidimensional functional diversity indices for a multifaceted framework in functional ecology. Ecology, 89, 2290-2301. https://doi. org/10.1890/07-1206.1
Voigt, W., Perner, J., Davis, A. J., Eggers, T., Schumacher, J., Bährmann, R., ... Sander, F. W. (2003). Trophic levels are differentially sensitive to climate. Ecology, 84, 2444-2453. https://doi.org/10.1890/02-0266

Vollstädt, M. G. R., Ferger, S. W., Hemp, A., Howell, K. M., Töpfer, T, Böhning-Gaese, K., \& Schleuning, M. (2017). Direct and indirect effects of climate, human disturbance and plant traits on avian functional diversity. Global Ecology and Biogeography, 26, 963-972. https://doi.org/10.1111/geb.12606

Walker, B., Stotz, D. F., Pequeño, T., \& Fitzpatrick, J. W. (2006). Birds of the Manu Biosphere Reserve. Fieldiana Zoology, 110, 23-49. https:// doi.org/10.3158/0015-0754(2006)110[23:botmbr]2.0.co;2

Webb, J. K., \& Shine, R. (1993). Prey-size selection, gape limitation and predator vulnerability in Australian blindsnakes (Typhlopidae). Animal Behaviour, 45, 1117-1126. https://doi.org/10.1006/anbe.1993.1136

Weinstein, B. G., \& Graham, C. H. (2017). Persistent bill and corolla matching despite shifting temporal resources in tropical hummingbird-plant interactions. Ecology Letters, 20, 326-335. https://doi. org/10.1111/ele.12730

Wheelwright, N. T. (1985). Fruit-size, gape width, and the diets of fruiteating birds. Ecology, 66, 808-818. https://doi.org/10.2307/1940542

\section{BIOSKETCH}

Larissa Nowak is interested in how functional traits shape biotic interactions and in how functional diversity of ecological communities might change under climate change in tropical ecosystems. This study is part of her dissertation at SBiK-F and Goethe University and has resulted from an ongoing collaboration between SBiK-F, IBED and ZFMK.

Author contributions: L.N., W.D.K., I.M.A.B. and M.S. developed the research ideas; L.N., W.D.K., I.M.A.B. and M.S. conceived the modelling approach with input from D.M.D. and K.B.G.; L.N., I.M.A.B., D.M.D. and T.T. compiled distribution and trait data; L.N. analysed the data and wrote the first draft of the manuscript, and all authors commented on the final manuscript.

\section{SUPPORTING INFORMATION}

Additional supporting information may be found online in the Supporting Information section at the end of the article.

How to cite this article: Nowak L. Kissling WD. Bender IMA, et al. Projecting consequences of global warming for the functional diversity of fleshy-fruited plants and frugivorous birds along a tropical elevational gradient. Divers Distrib. 2019;25:1362-1374. https://doi.org/10.1111/ddi.12946 


\section{Supplementary material}

\section{SI: Methods}

\section{S1: Museum specimen (frugivorous birds)}

If available, specimens from Peruvian localities were measured, but specimens from neighbouring countries (mainly Bolivia) were used, if specimens from Peru were not available. For 222 species, traits were measured from specimens from Peru and Bolivia. The list below gives specimen number and collection acronyms for measured bird specimen: FMNH - Field Museum of Natural History Chicago, LSUZM - Zoological Museum of the Louisiana State University Baton Rouge, BMNH - The Natural History Museum (Bird Group) Tring, NMB - Museum of Natural History Basel, SMF Senckenberg Museum Frankfurt, SNSD - Senckenberg Natural History Collections Dresden, ZFMK - Zoological Research Museum A. Koenig Bonn (Dehling, Fritz, et al., 2014). List of specimens provided separately in digital supporting information.

\section{S2: Temperature projections, tropospheric lapse rate and vertical distances}

We extracted raster data on current and future annual mean temperature for our study region from WorldClim (Hijmans et al. 2005) at a $2.5 \mathrm{~min}$ spatial resolution. WorldClim provides interpolations of observed current climate data representative of the time span 1960-1990. Data on future annual mean temperature are based on the IPCC's fifth assessment report (Stocker et al. 2014). We used projected mean annual temperature for the year 2080 (averaged over 2061-2080), according to five general circulation models (CCSM4, HadGEM2-ES, MIROC 5, MRI-CGCM and NorESM) and the representative concentration pathway (RCP) 8.5. We calculated anomalies in annual mean temperature as the difference between the current and the projected future annual mean temperature for each grid cell.

We took mean annual tropospheric lapse rate values for the period 1948-2001 from Mokhov \& Akperov (2006) at a $2.5^{\circ}$ spatial resolution. We interpolated the lapserate data bilinearly to match the $2.5 \mathrm{~min}$ spatial resolution of the temperature data. Each of our seven elevations was located in a separate grid cell. Therefore, we were able to derive elevation-specific temperature anomalies and lapse rates. 


\section{SII: Figures}

a)

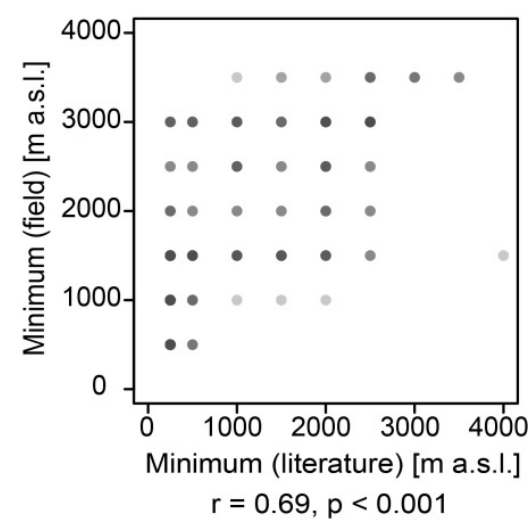

b)

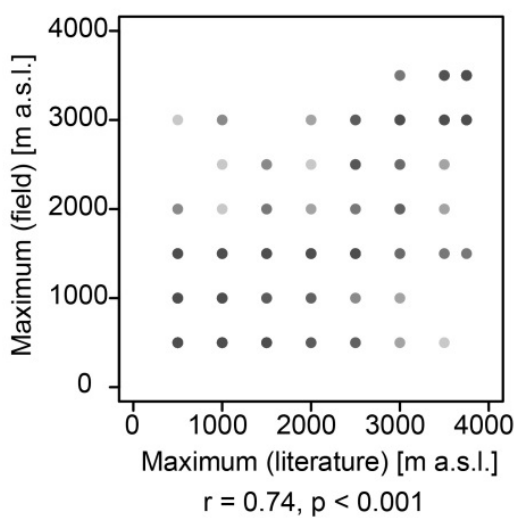

c)

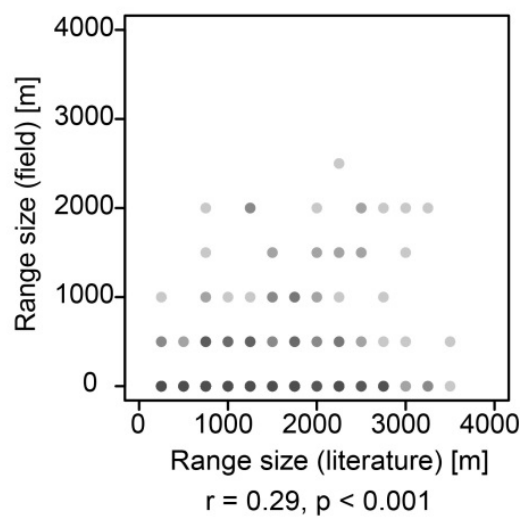

Figure S1: (a) Minimum elevation, (b) maximum elevation and (c) elevational range of plant species according to the literature (Brako \& Zarucchi 1993; Tropicos 2017) and based on local data from 1-ha sampling area per elevation (Dehling et al. 2014b). Given are the coefficient and $p$-value of a Pearson correlation that was performed to test for a relationship between the literature and the field data. Shading of points indicates the number of plant species.

a)

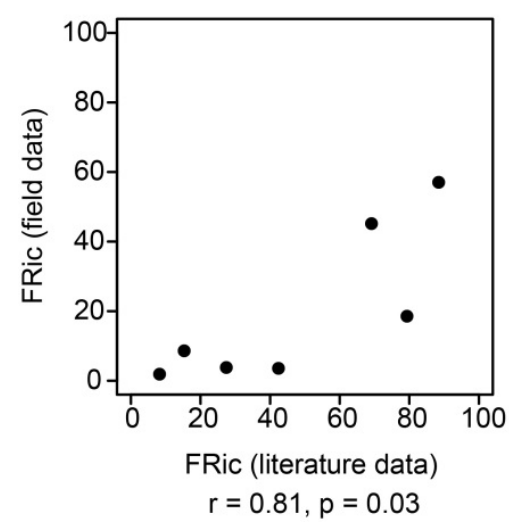

b)

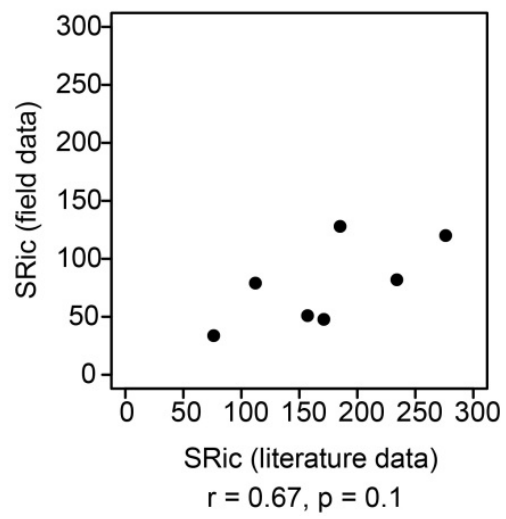

Figure S2: Relationship of (a) functional richness and (b) species richness of current communities of fleshy-fruited plants computed based on literature data (Brako \& Zarucchi 1993; Tropicos 2017) and local data from 1-ha sampling area per elevation (Dehling et al. 2014b). Given are the coefficient and p-value of a Pearson correlation. 


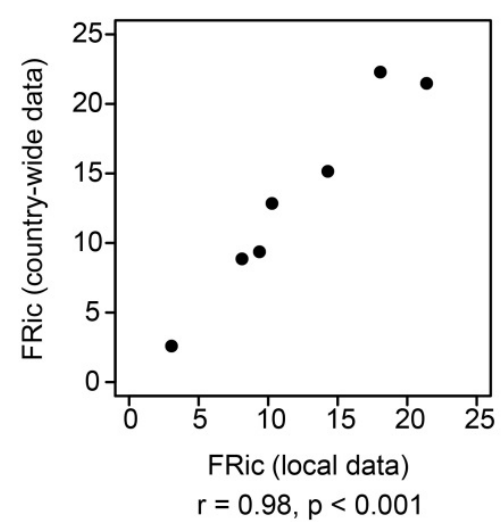

b)

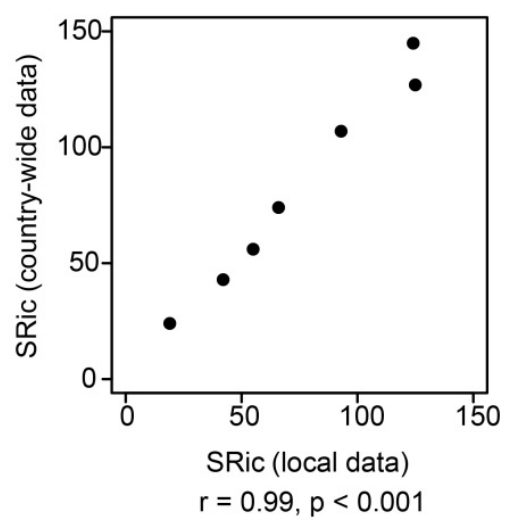

Figure S3: Relationship of (a) functional richness and (b) species richness of current communities of frugivorous birds computed based on local checklists (Walker et al. 2006; Merkord 2010; Dehling et al. 2013) and a country-wide guide book (Schulenberg et al. 2010). Given are the coefficient and $p$-value of a Pearson correlation.

a)
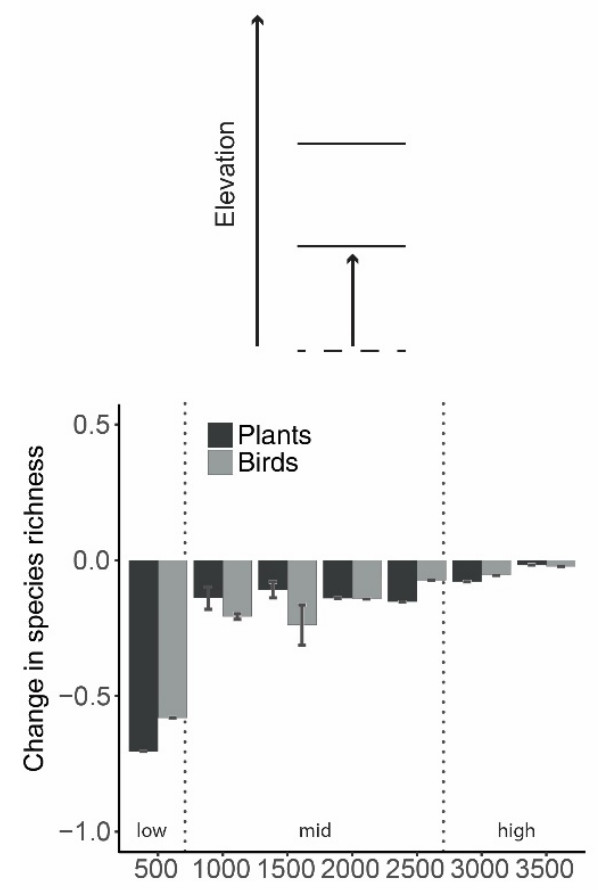

b)

Range expansion
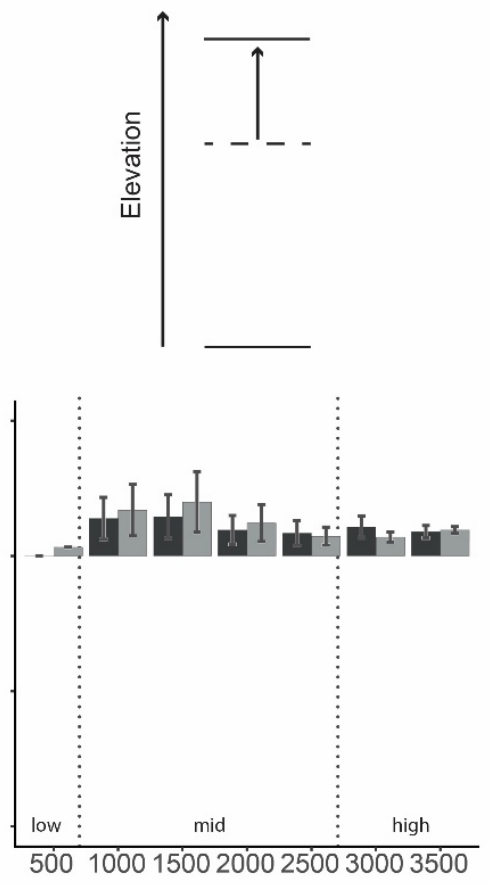

c)

Range shift
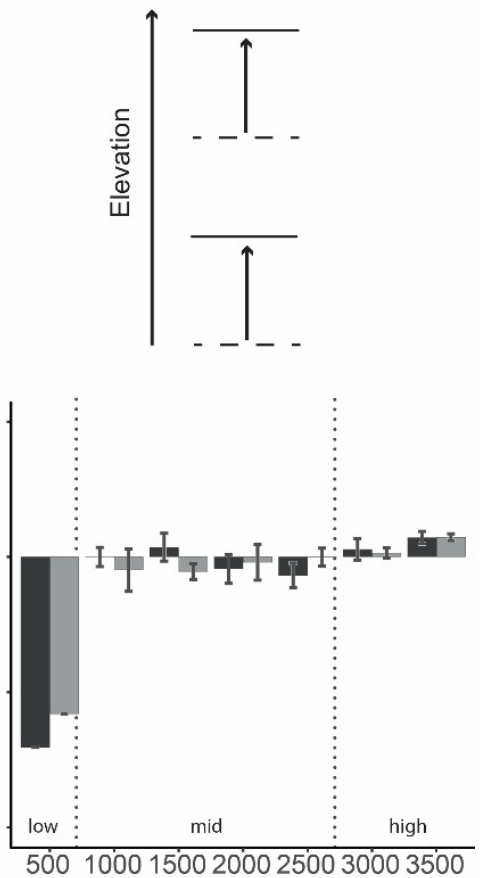

Elevation [m a.s.I.]

Figure S4: Projected changes in fleshy-fruited plant (dark grey) and frugivorous bird (medium grey) species richness under RCP 8.5 for the year 2080. We applied three vertical dispersal scenarios for future projections of plant and bird communities along the Manú gradient, i.e. (a) range contraction, (b) range expansion and (c) range shift. Changes were computed as future species richness minus current species richness. Given are mean values and standard deviation (error bars) over five general circulation models (CCSM4, HadGEM2-ES, MIROC 5, MRI-CGCM and NorESM). Dashed lines indicate low, mid and high elevations. 
a)

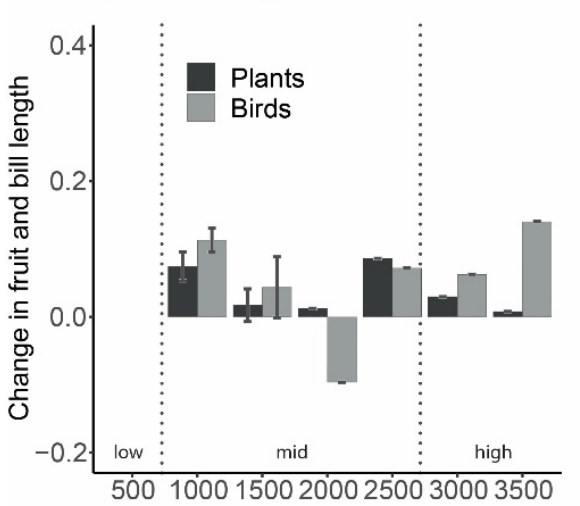

d)

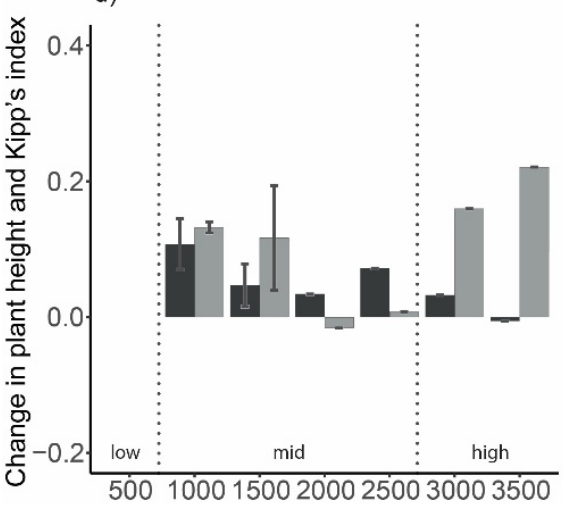

g)

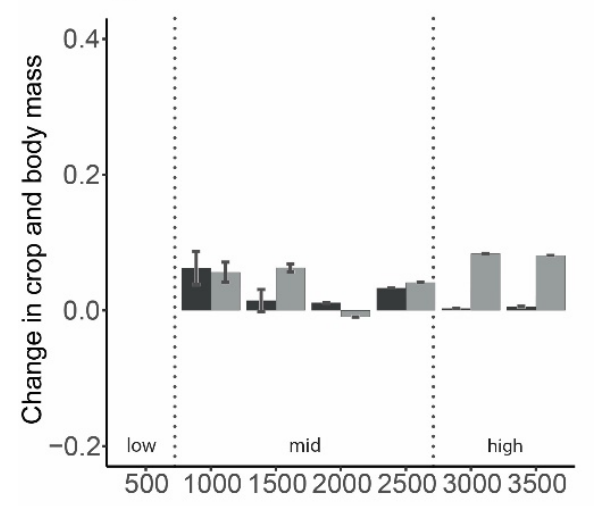

b)

Range expansion

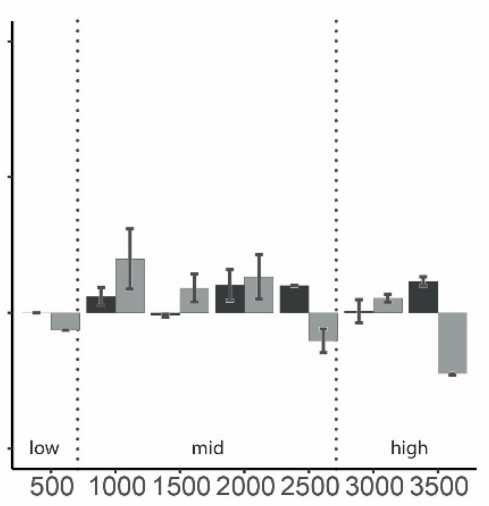

e)

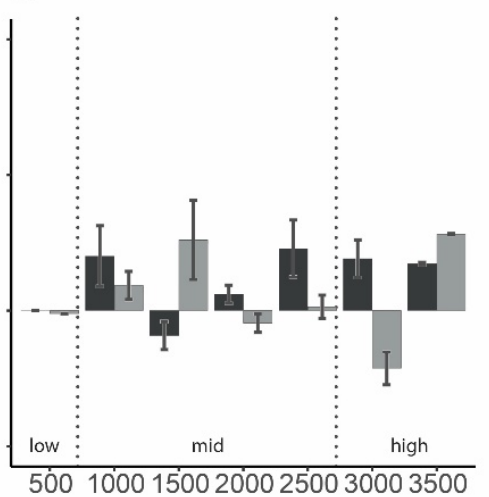

h)

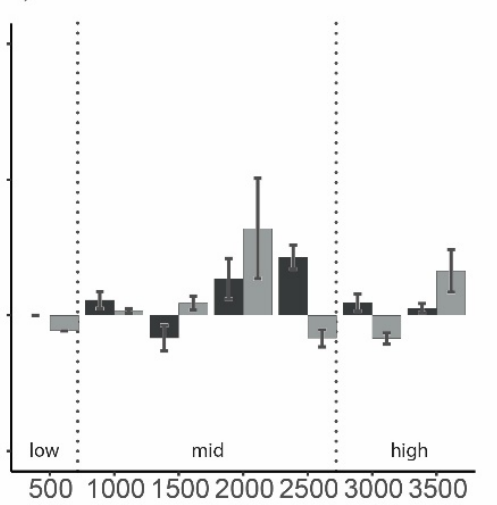

c) Range shift

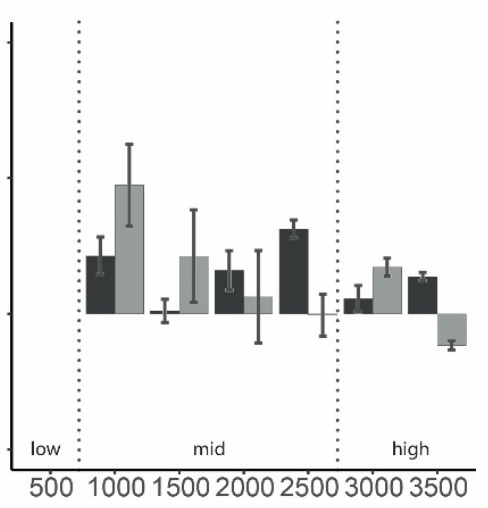

f)

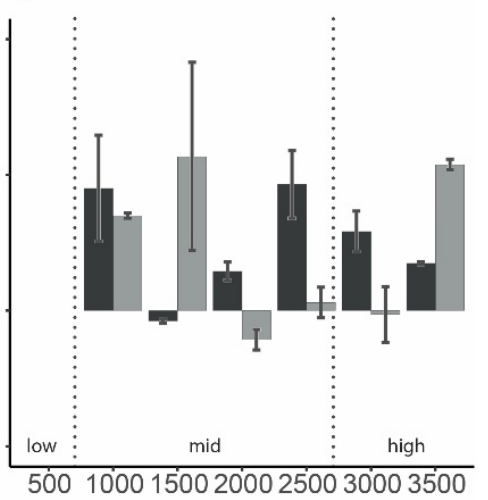

i)

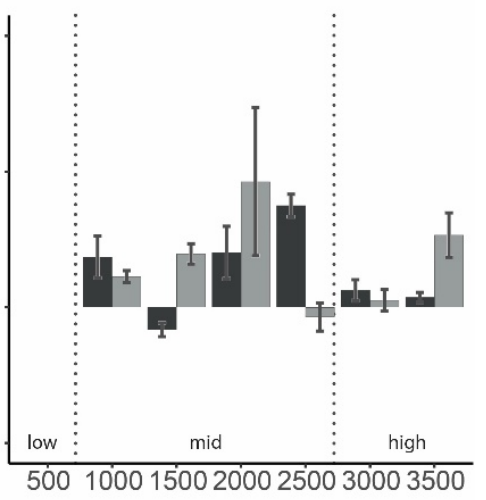

Elevation [m a.s.I.]

Figure S5: Projected changes in fleshy-fruited plant (dark grey) and frugivorous bird (medium grey) functional identity under RCP 8.5 for the year 2080 and according to three vertical dispersal scenarios (range contration, range expansion and range shift). Shown are changes in $(\mathrm{a}-\mathrm{c})$ bill width and fruit width, $(\mathrm{d}-\mathrm{f})$ plant height and Kipp's index and ( $\mathrm{g}-\mathrm{i})$ crop mass and avian body mass. Changes were computed as future functional identity minus current functional identity. Given are mean values and standard deviation (error bars) over five general circulation models (CCSM4, HadGEM2-ES, MIROC 5, MRI-CGCM and NorESM). Dashed lines indicate low, mid and high elevations. Functional identity of lowland communities could not be quantified under range contraction and shift scenarios because projected species richness is zero. 
a)

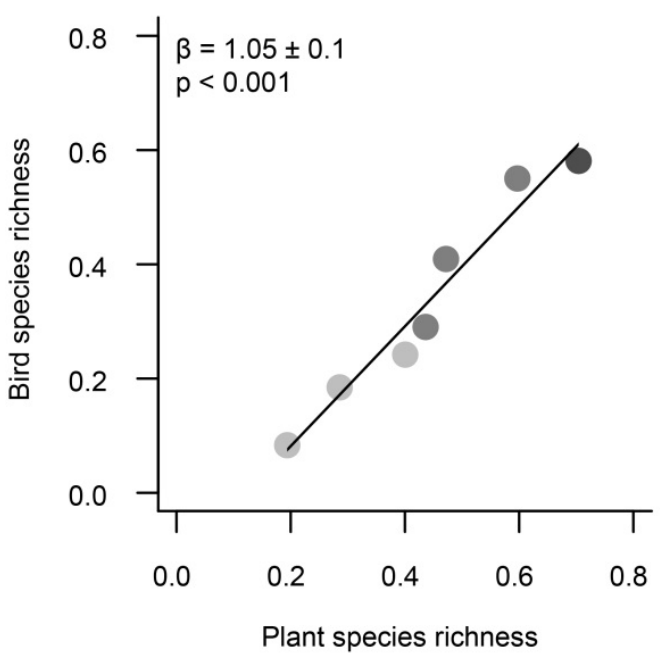

c)

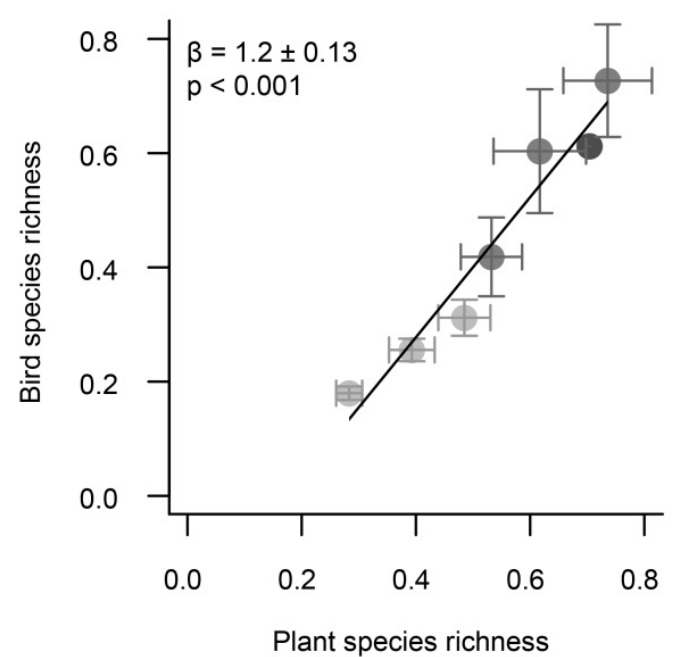

b)

Range contraction

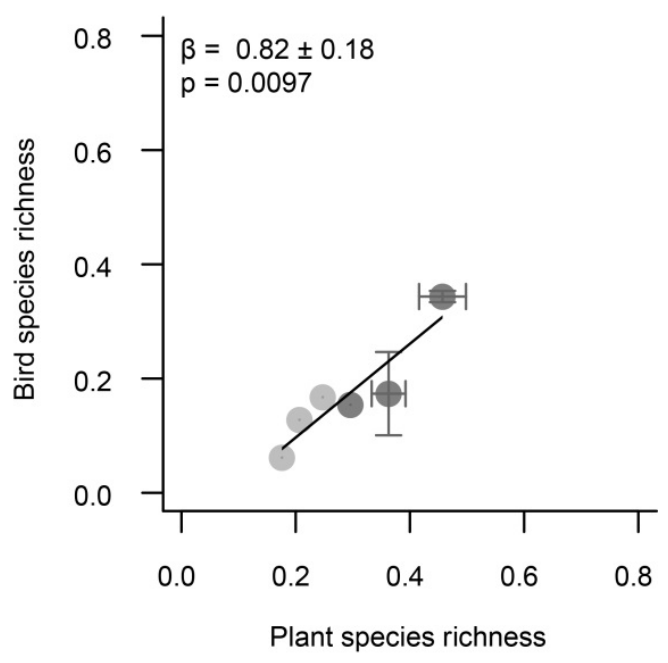

d) Range shift

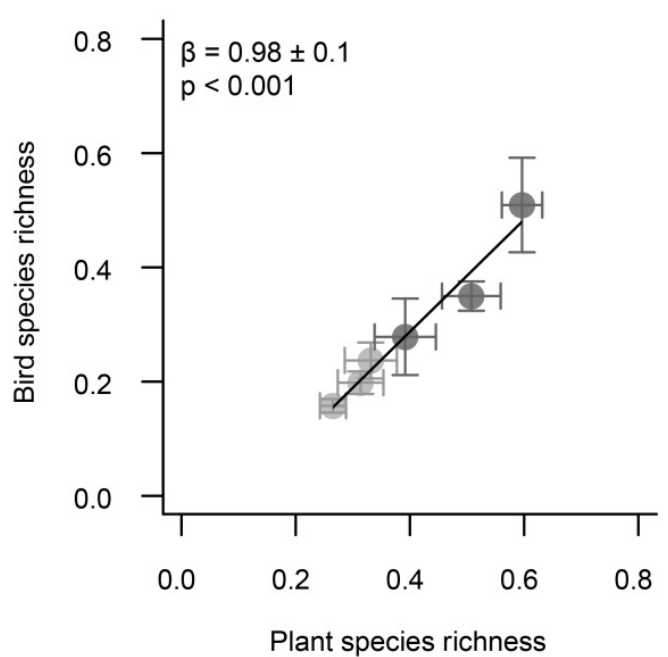

Figure S6: Associations between plant and bird species richness under (a) current conditions and under scenarios of (b) range contraction, (c) range expansion and (d) range shift. Dots indicate the mean value and error bars the standard deviation across five GCMs (CCSM4, HadGEM2-ES, MIROC 5, MRI-CGCM and NorESM). Horizontal error bars refer to plants, vertical error bars refer to birds. Given are slope estimates $(\beta) \pm$ standard errors and the p-values of linear models of bird functional richness against plant functional richness. Regression lines are represented as solid lines (for $p<0.05$ ) and dashed lines (for $0.05<p<0.1$ ). Low, mid and high elevations are coloured in dark, medium and light grey, respectively. 

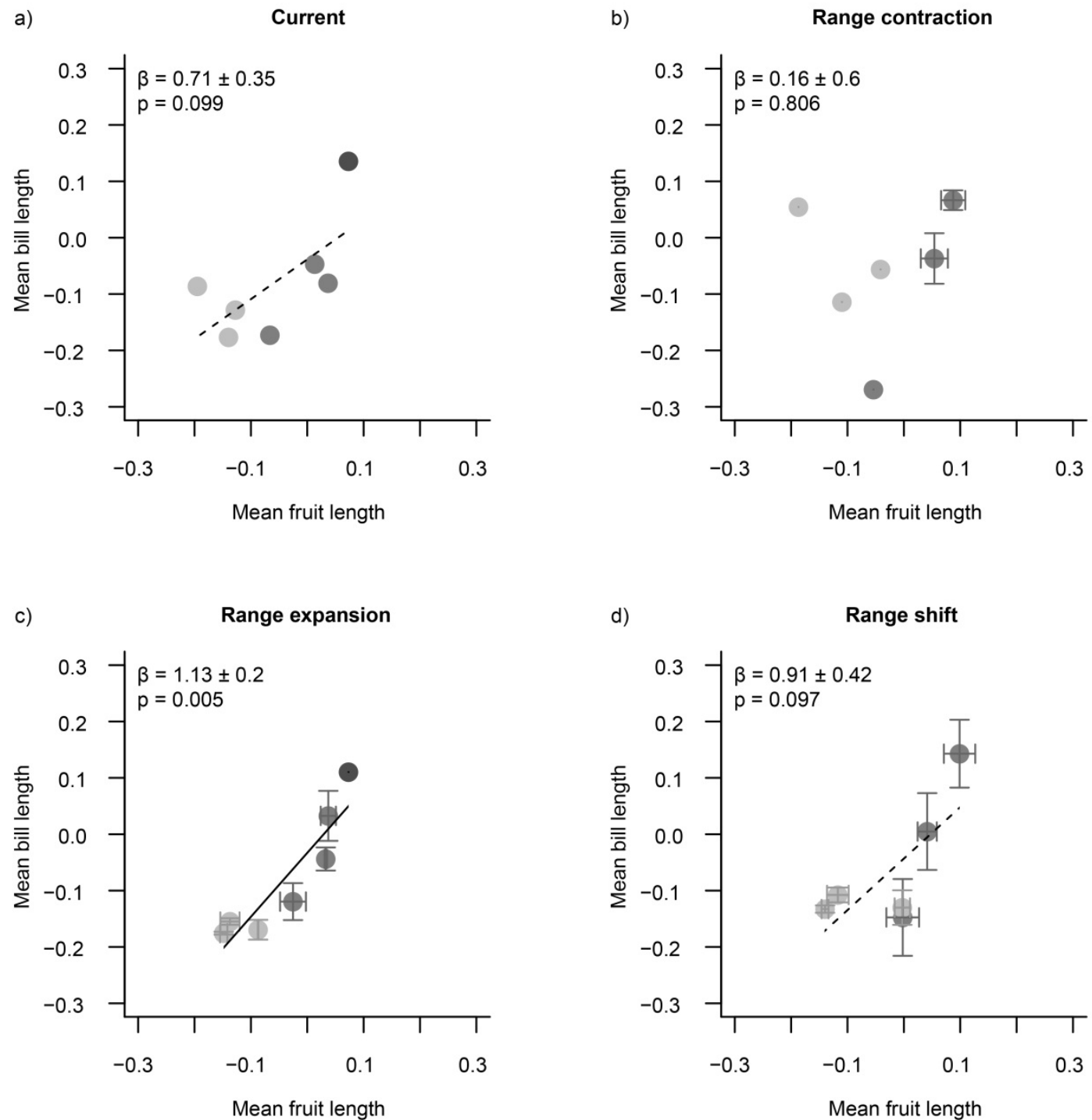

Figure S7: Associations between mean fruit length and mean bill length under (a) current conditions and under scenarios of (b) range contraction, (c) range expansion and (d) range shift. Dots indicate the mean value and error bars the standard deviation across five GCMs (CCSM4, HadGEM2-ES, MIROC 5, MRI-CGCM and NorESM). Horizontal error bars refer to plants, vertical error bars refer to birds. Given are slope estimates $(\beta) \pm$ standard errors and the $p$-values of linear models of bird functional richness against plant functional richness. Regression lines are represented as solid lines (for $p<0.05$ ) and dashed lines (for $0.05<p<0.1$ ). Low, mid and high elevations are coloured in dark, medium and light grey, respectively. 
a)

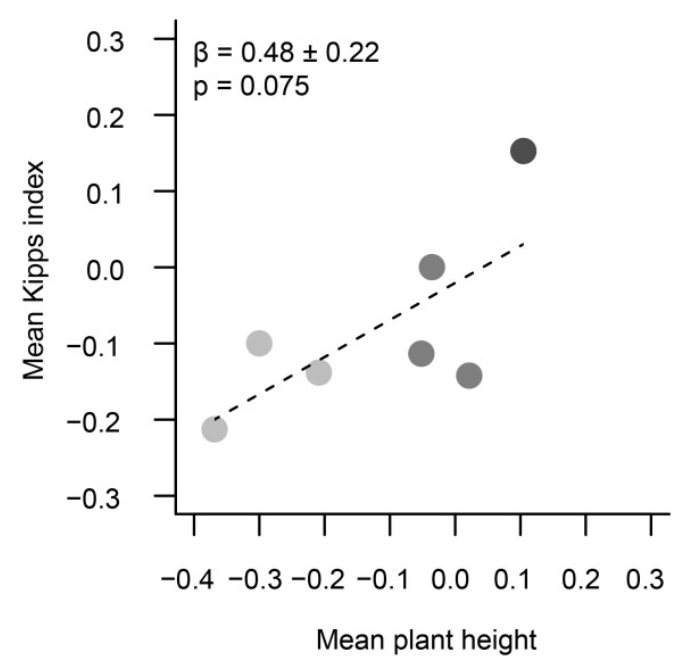

c)

Range expansion

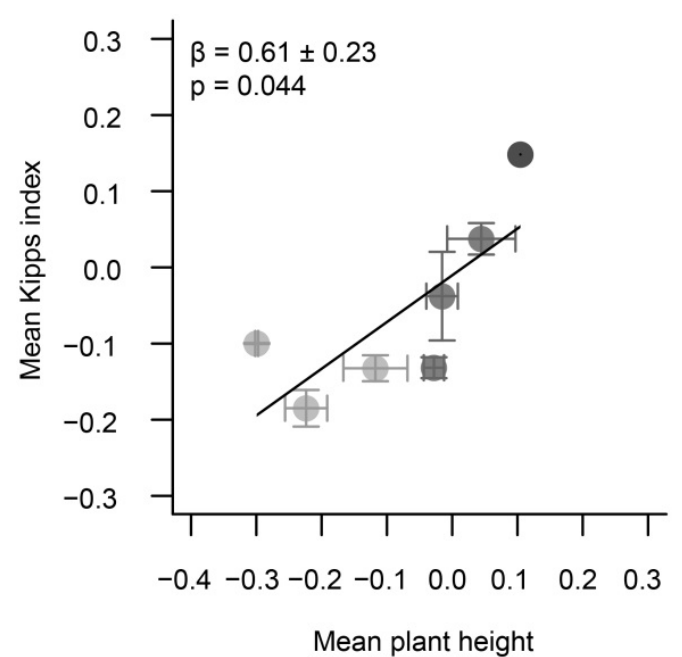

b)

Range contraction

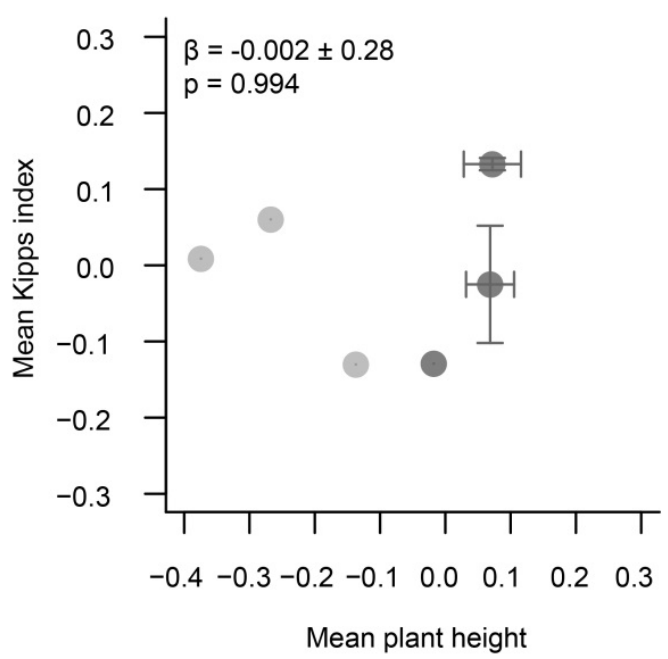

d)

Range shift

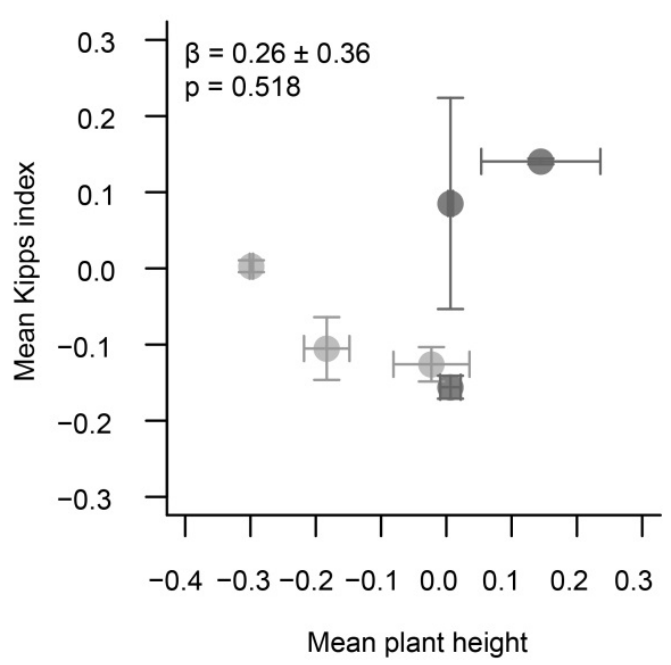

Figure S8: Associations between mean plant height and mean Kipp's index under (a) current conditions and under scenarios of (b) range contraction, (c) range expansion and (d) range shift. Dots indicate the mean value and error bars the standard deviation across five GCMs (CCSM4, HadGEM2-ES, MIROC 5, MRI-CGCM and NorESM). Horizontal error bars refer to plants, vertical error bars refer to birds. Given are slope estimates $(\beta) \pm$ standard errors and the $p$-values of linear models of bird functional richness against plant functional richness. Regression lines are represented as solid lines (for $p<0.05$ ) and dashed lines (for $0.05<p<0.1$ ). Low, mid and high elevations are coloured in dark, medium and light grey, respectively. 
a)

Current

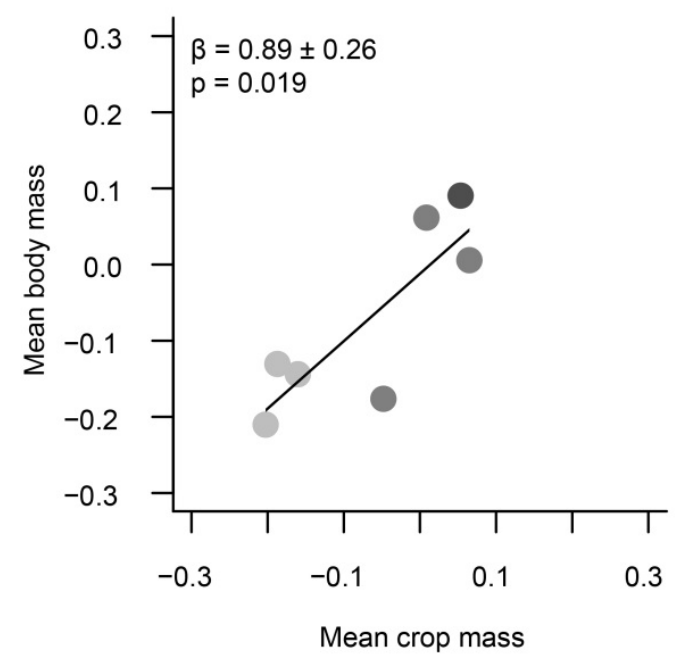

c)

Range expansion

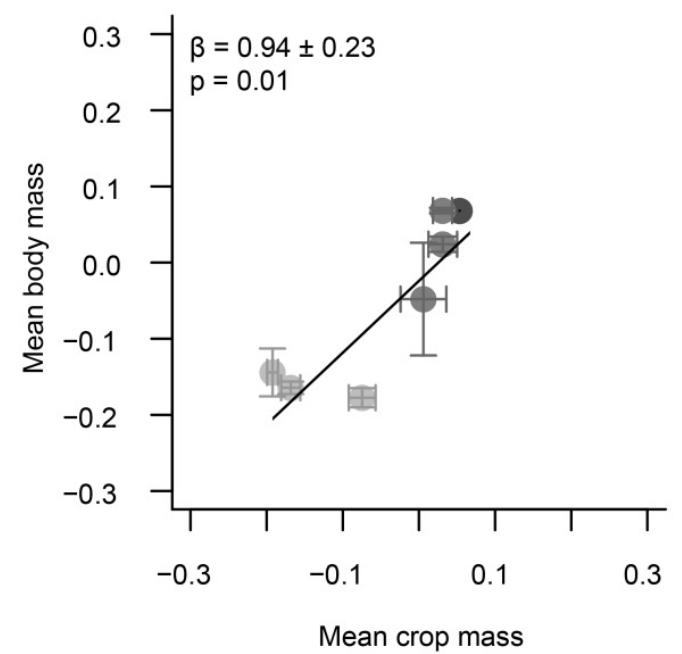

b)

Range contraction

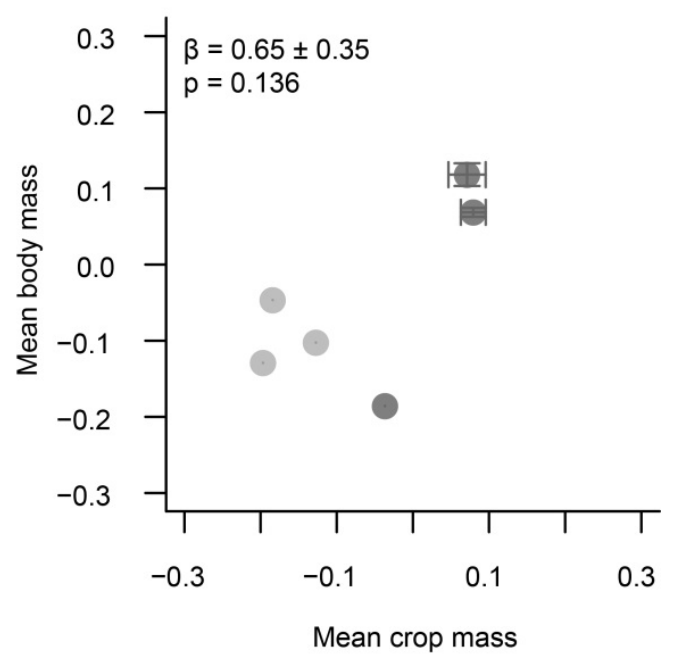

d)

Range shift

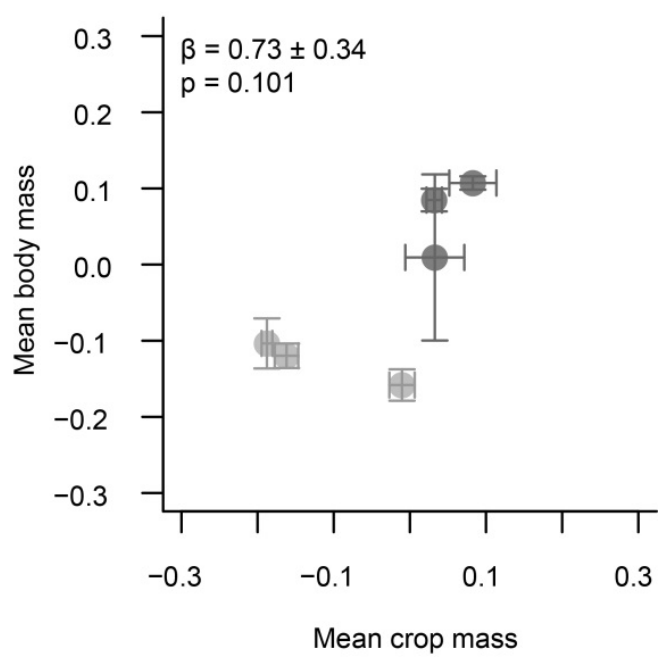

Figure S9: Associations between mean plant crop mass and mean avian body mass under (a) current conditions and under scenarios of (b) range contraction, (c) range expansion and (d) range shift. Dots indicate the mean value and error bars the standard deviation across five GCMs (CCSM4, HadGEM2-ES, MIROC 5, MRI-CGCM and NorESM). Horizontal error bars refer to plants, vertical error bars refer to birds. Given are slope estimates $(\beta) \pm$ standard errors and the $p$-values of linear models of bird functional richness against plant functional richness. Regression lines are represented as solid lines (for $p<0.05$ ) and dashed lines (for $0.05<p<0.1$ ). Low, mid and high elevations are coloured in dark, medium and light grey, respectively. 


\section{References}

Brako, L., \& Zarucchi, J. L. (1993). Catalogue of the flowering plants and Gymnosperms of Peru. Catálogo de las Angiospermas y Gimnospermas del Perú. Monographs in systematic botany from the Missouri Botanical Garden 45.

Dehling, D. M., Fritz, S. A., Töpfer, T., Päckert, M., Estler, P., Böhning-Gaese, K., \& Schleuning, M. (2014). Functional and phylogenetic diversity and assemblage structure of frugivorous birds along an elevational gradient in the tropical Andes. Ecography, 37, 1047-1055. http://doi.org/10.1111/ecog.00623

Dehling, D. M., Sevillano, C. S., \& Morales, L. V. (2013). Upper and lower elevational extremes of Andean birds from south-east Peru. Boletín Informativo, 8, 32-38.

Dehling, D. M., Töpfer, T., Schaefer, H. M., Jordano, P., Böhning-Gaese, K., \& Schleuning, M. (2014). Functional relationships beyond species richness patterns: trait matching in plant-bird mutualisms across scales. Global Ecology and Biogeography, 23, 1085-1093. http://doi.org/10.1111/geb.12193

Hijmans, R. J., Cameron, S. E., Parra, J. L., Jones, P. G., \& Jarvis, A. (2005). Very high resolution interpolated climate surfaces for global land areas. International Journal of Climatology, 25, 1965-1978. http://doi.org/10.1002/joc.1276

Merkord, C. L. (2010). Seasonality and elevational migration in an Andean bird community. University of Missouri-Columbia.

Mokhov, I. I., \& Akperov, M. G. (2006). Tropospheric lapse rate and its relation to surface temperature from reanalysis data. Izvestiya, Atmospheric and Oceanic Physics, 42, 430-438. http://doi.org/10.1134/s0001433806040037

Schulenberg, T. S., Stotz, D. F., Lane, D. F., O’Neill, J. P., \& Parker III, T. A. (2010). Birds of Peru: revised and updated edition (Vol. 63). Princeton University Press. Stocker, T. F., Qin, D., Plattner, G.-K., Tignor, M. M. B., Allen, S. K., Boschung, J., ... Midgley, P. M. (2014). Climate Change 2013 - The Physical Science Basis. (Intergovernmental Panel on Climate Change, Ed.). Cambridge: Cambridge University Press. http://doi.org/10.1017/CBO9781107415324

Tropicos. (2017). Tropicos. Retrieved from http://www.tropicos.org

Walker, B., Stotz, D. F., Pequeño, T., \& Fitzpatrick, J. W. (2006). Birds of the Manu Biosphere Reserve. Fieldiana Zoology, 23-49. http://doi.org/10.3158/00150754(2006)110[23:botmbr]2.0.co;2 


\section{A3: Avian seed dispersal may be insufficient for plants to track future temperature change on tropical mountains.}

Authors: Larissa Nowak, Matthias Schleuning, Irene M. A. Bender, Katrin BöhningGaese, D. Matthias Dehling, Susanne A. Fritz, W. Daniel Kissling, Thomas Mueller, Eike Lena Neuschulz, Alex L. Pigot, Marjorie C. Sorensen, Isabel Donoso

Title: Avian seed dispersal may be insufficient for plants to track future temperature change on tropical mountains

Status: submitted

Author contributions:

(1) Concept and design

LN, 85\%;

MS, ID, in total $10 \%$;

ALP, WDK, in total $5 \%$

\section{(2) Conducting tests and experiments}

n. a.

\section{(3) Compilation of data sets and figures}

DMD identified plant species in the field, 100\%;

IMAB compiled plant elevational distributions from literature, $100 \%$;

DMD and IMAB measured plant morphological traits, in total $100 \%$;

DMD compiled bird elevational distributions from literature \& field observations, $100 \%$;

LN compiled bird body mass data from literature, 100\%;

DMD measured bird morphological data, $100 \%$;

IMAB derived local climate data from literature, 100\%;

LN downloaded and processed bioclimatic data, 100\%;

LN derived lapse-rate data from literature and processed the data, $100 \%$;

LN downloaded and processed slope data, 100\%;

LN prepared all data for analyses, $100 \%$;

LN prepared all figures and tables, $100 \%$

\section{(4) Analysis and interpretation of data}

ID, MS developed the trait-based interaction model, $100 \%$,

MCS, ID, TM, MS, ELN developed the trait-based seed-dispersal model, $100 \%$,

LN developed the workflow and adapted the models for her study, $100 \%$,

LN simulated interaction events and computed seed dispersal distances, $100 \%$;

LN computed projected vertical temperature shifts, required horizontal dispersal distances and number of required long-distance dispersal events, 100\%;

LN interpreted the results, $90 \%$ with input from MS, IMAB, KBG, DMD, SAF, WDK, TM, ELN, ALP, MCS, ID, in total $10 \%$

\section{(5) Drafting of manuscript}

LN, $90 \%$;

MS, ID, in total $5 \%$;

IMAB, KBG, DMD, SAF, WDK, TM, ELN, ALP, MCS, in total 5\% 


\title{
Avian seed dispersal may be insufficient for plants to track future temperature change on tropical mountains
}

Running title: Avian seed dispersal and climate change

\begin{abstract}
Aim: Climate change causes species' range shifts globally. However, terrestrial plant species often lag behind temperature shifts and it is unclear to what extent animaldispersed plants can track climate change. Here, we quantify the ability of birddispersed plant species to track future temperature change on a tropical mountain.

Location: Tropical elevational gradient (500-3500 m a.s.I.) in the Manú biosphere reserve, southeast Peru
\end{abstract}

Time period: 1960-1990 to 2061-2080

Taxa: Fleshy-fruited plants, avian frugivores

Methods: With a combination of simulation models building on functional traits of avian frugivores and fruiting plants, we quantified the number of long-distance dispersal (LDD) events that woody plant species would require to track projected temperature shifts on a tropical mountain by the year 2070. We applied this approach to 343 woody, bird-dispersed plant species.

Results: Our simulations suggest that the LDD ability of most plant species may be insufficient, as they were projected to require several LDD events to track projected vertical temperature shifts until 2070. For the majority of plant species studied here, this is an unlikely scenario given the longevity and slow response times of woody plants. Our simulations also reveal that bird-dispersed plants strongly differ in their ability to track climate change. Large-fruited and canopy plants have a higher ability to track future temperature changes. Furthermore, we found that the ability of plant species to track future temperature changes increased in simulations with a low degree of trait matching between plants and birds.

Main conclusion: Our study illustrates how functional traits can be used to quantify plant species' dispersal ability and how trait-based models can inform on whether plant species may be able to track rapid climate change. The increasing availability of functional trait data of plants and birds globally will allow parameterization of similar models for many other seed-dispersal systems. 


\section{Keywords}

global warming, range shifts, biotic interactions, functional traits, biodiversity projections, long-distance dispersal, trait-based simulation model

\section{Introduction}

Climate change is causing a rapid redistribution of biodiversity globally ( $\mathrm{Pecl}$ et al. 2017), with species shifting towards higher latitudes and elevations as they track suitable climatic conditions (Feeley et al. 2011, Brommer et al. 2012). Species are expected to differ widely in the pace at which they can shift their geographic range, with marine species tracking changing climates more closely than species on land (Lenoir et al. 2020). Among terrestrial plants, recent range shifts appear to be lagging behind changes in temperature (Feeley et al. 2011, Lenoir et al. 2020), raising the risk that plant species will be unable to shift their ranges sufficiently rapidly to reach suitable climates. However, quantitative estimates of plant species' ability to keep pace with climate change are largely lacking (but see Naoe et al. 2016, González-Varo et al. 2017).

Quantifying species' dispersal is key for predicting species' ability to track rapid climate change (Schleuning et al. 2020, Sales et al. 2021). The dispersal ability of plant species relates to present-day range filling (Estrada et al. 2015) and range expansion during past climate change (Hampe 2011). Long-distance dispersal is particularly crucial for plant species under climate change because it determines the maximum distance a propagule can be dispersed (Cain et al. 2000). The dispersal ability of plant species is determined by their traits (Chen et al. 2019). Seed dispersal distances are often positively related to plant height and vary with seed size (Thomson et al. 2011, Chen et al. 2019). In the tropics, plant species show a greater range of seed sizes and can grow taller than in temperate regions (Moles et al. 2009). However, empirical dispersal estimates only exist for a small number of tropical plant species (Chen et al. 2019). Consequently, it remains little understood how the dispersal ability of tropical plants varies with their traits, especially because seed dispersal of the majority of woody plant species in the tropics depends on animals, in particular birds (Jordano 2014, Chen et al. 2017).

Fleshy-fruited plants and avian frugivores are linked in complex seed-dispersal networks and the interactions in these networks are influenced by species' traits. Trait matching (i.e., the compatibility of species' traits) is a general phenomenon 
determining species' interactions in seed-dispersal networks (Burns 2013, Bender et al. 2018). The degree of trait matching (i.e., how closely the trait values of two interacting species match) varies in empirical seed-dispersal networks, likely reflecting the flexibility of frugivores in switching between fruit resources (Bender et al. 2017, 2018). For instance, large-billed frugivores, despite preferring large fruits, can feed on a wide range of fruit sizes, whereas small-billed species are usually constrained to feed on small fruits (Wheelwright 1985). A recent simulation study suggests that a low degree of trait matching can lead to larger dispersal distances of bird-dispersed plant communities because large birds contribute to the dispersal of more plant species in such networks (Sorensen et al. 2020). However, the impact of the degree of trait matching on the ability of bird-dispersed plants to track rapid climate change remains untested.

Functional traits can be used to quantify and compare the dispersal ability among bird-dispersed plants because traits determine both the identity of avian dispersers and the seed-dispersal distances provided to plants (Schleuning et al. 2020). Here, we integrate two recently developed trait-based models to compare the long-distance dispersal (LDD) ability of plant species (Fig. $1 \mathrm{a}, \mathrm{b}$ ) to projected vertical temperature shifts under different future greenhouse gas emission scenarios (Fig. $1 \mathrm{c}$ ). First, we apply a trait-based interaction model to simulate seed-dispersal interactions between plant and bird species considering different degrees of trait matching between bill size and fruit size (Fründ et al. 2016, Donoso et al. 2017; Fig. 1 a). Second, we apply a traitbased seed-dispersal model based on allometric scaling relationships between avian body mass, gut passage time and flight speed to estimate dispersal distances for each interaction event (Sorensen et al. 2020; Fig. 1 b). Finally, to compare the estimated dispersal distance of a plant species to the distance that temperatures are projected to shift vertically (Fig. $1 \mathrm{c}$ ), we quantify the number of LDD events a plant species would require to track temperature change by 2070 (Fig. 1 d).

With this new integrative simulation approach, we assess the LDD ability of 343 bird-dispersed, woody plant species along a tropical elevational gradient in the Andes of south-east Peru. We evaluate i) how the projected number of LDD events required by 2070 would vary with the degree of trait matching, and (ii) to what extent the projected number of LDD events required by 2070 would differ among plant species with different traits. We hypothesize (i) that plant species require fewer LDD events if the degree of trait matching is low because dispersal distances of bird-dispersed plants 
are related to the degree of trait matching in seed-dispersal networks (Sorensen et al. 2020). We further hypothesize (ii) that large-fruited species require fewer LDD events than small-fruited species because large-fruited species interact preferentially with large-billed and large-sized frugivores which disperse seeds farther (Jordano et al. 2007, Burns 2013). A similar mechanism may apply to canopy plants if plant height is positively related to fruit size (Shanahan and Compton 2001).
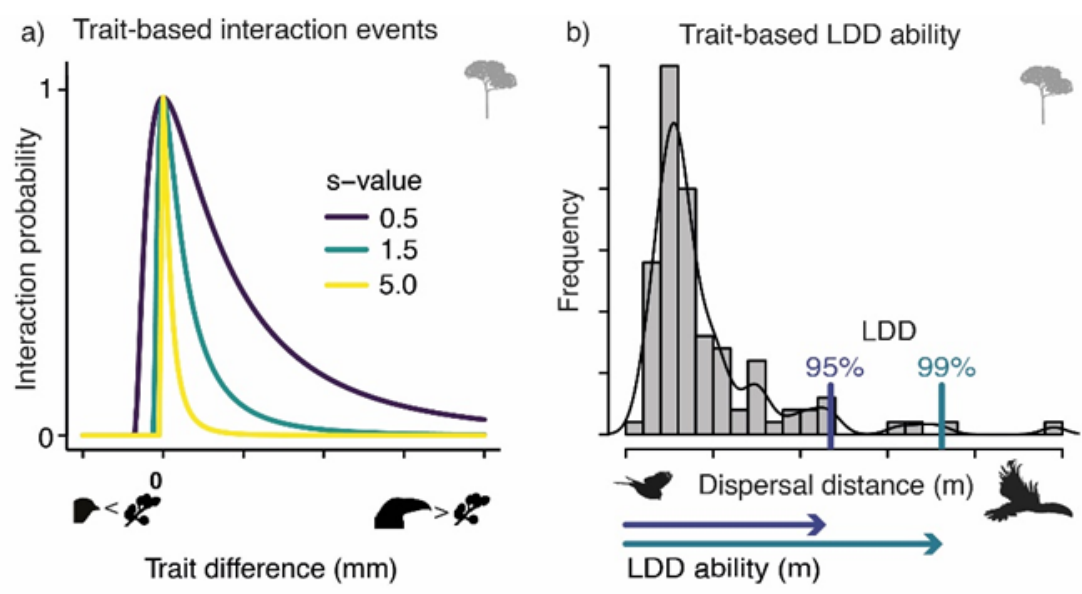

c) Projected vertical temperature shift

d)

Projected number of LDD events
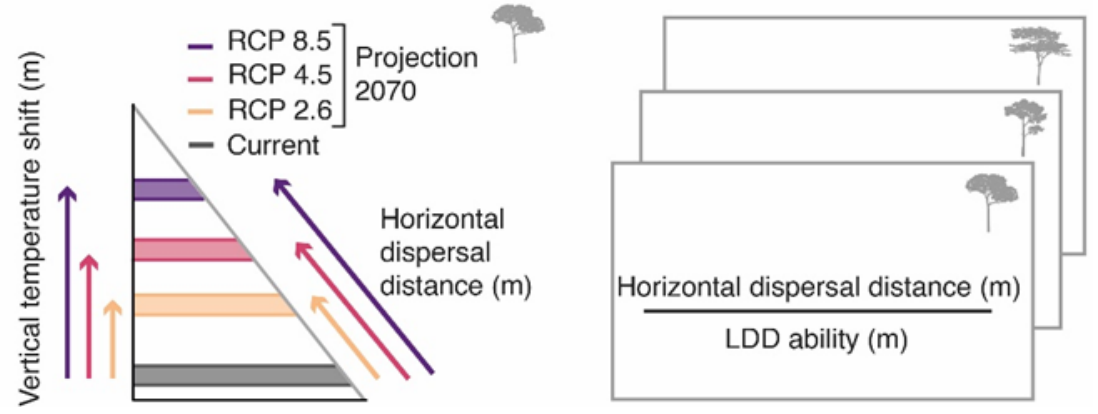

Figure 1: Trait-based projections of the number of long-distance dispersal (LDD) events. (a) Trait-matching between the fruit width of a plant and the bill width of potential avian dispersers informs simulations of seed-dispersal interaction probabilities (Donoso et al. 2017). The degree of trait matching was varied according to a trait-matching parameter (s-value, three settings as shown in legend). (b) Simulations of dispersal distance are based on allometric relationships of the gut passage time and the flight speed, whereby dispersal distance is positively related to avian body mass (Sorensen et al. 2020). We simulated a dispersal distance for each interaction event between a bird and a plant species and considered the 95th and the 99th percentile of the frequency distribution of all dispersal distances as two alternative proxies for the LDD ability of that plant species (95\% and 99\% LDD, respectively). (c) The projected vertical temperature shift estimates the distance by which the current realized temperature range of a plant species along an elevational gradient is projected to shift vertically (Nowak et al. 2019). Here, we estimated projected vertical temperature shifts until 2070 for three representative concentration pathways (RCP) representing a strong mitigation (RCP2.6), a moderate mitigation (RCP4.5), and a high emissions scenario (RCP8.5). We computed the required horizontal dispersal distance 
as vertical temperature shift $(\mathrm{m})$ divided by the sine of the mean slope $\left(^{\circ}\right)$. (d) The number of LDD events that a plant species requires to track projected vertical temperature shifts by 2070 can be estimated as the ratio of the required horizontal dispersal distance and the simulated LDD ability of that plant species.

\section{Methods}

\section{Study system}

Our study system is an elevational gradient (250 m a.s.l. to $3750 \mathrm{~m}$ a.s.I) in the Manú biosphere reserve in south-east Peru. The gradient is covered by lowland and montane rain forest, cloud forest and elfin forest (Patterson et al. 1998). All forest types are characterized by high precipitation (minimum annual precipitation along the gradient about $1560 \mathrm{~mm}$ ), while mean annual temperature declines with increasing elevation from about $24.3^{\circ} \mathrm{C}$ at $500 \mathrm{~m}$ a.s.I. to $7.3^{\circ} \mathrm{C}$ at $3500 \mathrm{~m}$ a.s.l. (Girardin et al. 2010, 2013).

\section{Plant and bird species and their current elevational ranges}

To identify bird-dispersed woody plant species occurring along the Manú gradient, all plants with ripe fleshy fruits were collected within an area of 1 ha (divided into 10 plots of $20 \mathrm{~m} \times 50 \mathrm{~m}$ ) located at seven elevations every $500 \mathrm{~m}$ a.s.l. (distances between the plots were at least $200 \mathrm{~m}$; Dehling et al. 2014). In the field, the plants were identified as morphospecies, samples of each were taken and identified to species level with specimens from local herbaria. Each site was sampled once in the rainy season (December to March) and once in the dry season (June to September) between December 2009 and September 2011. The resulting plant species pool comprised 343 bird-dispersed, woody plant species. This included all woody species that were identified to species-level and for which trait and elevation data were available (Table S1). We derived current minimum and maximum elevation of the plant species across Peru at an elevational resolution of $500 \mathrm{~m}$ from literature (Brako and Zarucchi 1993), supplemented by data from the Tropicos website (2017; for about $3 \%$ of the analysed species).

We considered 219 frugivorous bird species that occur along the Manú gradient and consume fruits as a main part of their diet (Table S2; Dehling et al. 2014). This species pool excluded ground dwellers (the families Tinamidae, Odontophoridae, Psophidae, and the genus Mitu) because they have a distinct movement behaviour. It further excluded the few seasonal migrants that are not present year-round (Catharus ustulatus, Elaenia parvirostris, Elaenia spectabilis, Elaenia strepera, Pirange rubra, 
Turdus amaurochalinus, Tyrannus savanna, Tyrannus tyrannus). Current minimum and maximum elevation of each bird species along the Manú gradient at an elevational resolution of $50 \mathrm{~m}$ were derived from local checklists supplemented by field observations (Walker et al. 2006, Dehling et al. 2013). Elevational ranges were derived from year-round observations and thus account for rare elevational movements of bird species over the year.

\section{Morphological traits of birds and plants}

We collected data on avian bill width and body mass as well as on fruit width and plant height for all species considered in our study (Tables S1, S2). Bill width ( $\mathrm{mm}$ ) was measured on museum specimens following measurement protocols from Eck et al. (2011) and aiming at measuring at least two females and two males per species. Because body mass influences avian movement (Westcott and Graham 2000, Jordano et al. 2007), we additionally compiled data on avian body mass from literature (Dunning 2007, Table S2). Fruit width ( $\mathrm{mm}$ ) was measured on fruits collected during the field surveys on 20 fruits per plant species. Additionally, plant height $(\mathrm{m})$ was measured as distance from the ground to the top of the plant during the field surveys on all individuals in the plots (Table S1). Plant height and fruit width were weakly positively correlated ( $n$ $=343$ species, $r=0.36, p<0.001)$.

To test whether the projected number of LDD events until 2070 differs among plant species with different traits, we grouped the plant species into small- vs. largefruited species. We considered plant species within the smallest quartile of fruit width as small-fruited $(\leq 4.99 \mathrm{~mm}$ ) and the species within the largest quartile of fruit width as large-fruited ( $\geq 11.41 \mathrm{~mm}$ ). Similarly, we grouped plants into understory and canopy species, considering plant species within the smallest quartile of plant height as understory species $(\leq 3.29 \mathrm{~m})$ and the species within the largest quartile of plant height as canopy species $(\geq 9.62 \mathrm{~m})$.

In all analyses, we used species means of plant and bird traits. For one plant species, we had to use trait values from Ecuador (Quitián et al. 2019) and for 20 plant species (ca. $6 \%$ of the species pool) we used mean trait values at the genus level since species-level trait values were unavailable (Table S1). Interspecific trait variation was large (e.g., bill width varied from $5.12 \mathrm{~mm}$ to $38.76 \mathrm{~mm}$ and fruit width varied from 0.5 $\mathrm{mm}$ to $38.4 \mathrm{~mm}$ ), therefore we expect intraspecific trait variation to be of little relevance for our simulations. 


\section{Trait-based interaction probabilities and interaction events}

With a trait-based interaction model, we computed interaction probabilities between all 219 bird and 343 plant species based on species' trait matching, specifically the size difference between fruit width and bill width (Donoso et al. 2017; Fig. 1 a, S1). In this model, a size difference of zero (bill width = fruit width) yields a high interaction probability. A positive mismatch in trait values (bill width $>$ fruit width) decreases the interaction probability. A negative mismatch (bill width < fruit width) yields an interaction probability close to zero (Donoso et al. 2017) because small-billed birds are unable to feed on large fruits (Bender et al. 2017). The model allows adjusting the degree of trait matching with a trait-matching parameter (Fig. 1 a) which influences the specialization of the resulting seed-dispersal network (Fründ et al. 2016, Donoso et al. 2017). We considered three trait-matching parameter values representing a low, an intermediate and a high degree of trait matching ( $s=0.5,1.5,5.0$, respectively), resulting in a low, an intermediate and a high degree of network specialization $\left(H 2^{\prime}=0.09,0.29,0.49\right.$, respectively). This covers the range of specialization of empirical seed-dispersal networks, most of which show an intermediate degree of network specialization (Dalsgaard et al. 2017, Dugger et al. 2019).

In the simulations of interaction events, we did not consider how variation in the abundance of bird or plant species may influence interactions because independent data on abundance were not available. To account for species co-occurrences along the elevational gradient, we multiplied the matrix of interaction probabilities by a matrix of co-occurrences of the bird and the plant species. We considered an overlap of the current elevational ranges of plant and bird species along the Manú gradient as cooccurrence and removed interaction links corresponding to pairs of species that do not co-occur along the gradient. To simulate interaction events, we drew 100 interaction events per plant species based on the resulting interaction probabilities (with replacement). We ran ten independent iterations and simulated long-distance dispersal for each run.

\section{Trait-based simulations of long-distance dispersal}

With a trait-based seed-dispersal model, we simulated a seed-dispersal distance for each of the 100 interaction events per plant species (Sorensen et al. 2020, Donoso et al. 2020; Fig. 1 b). This seed-dispersal model is based on allometric relationships between avian body mass and avian gut passage time and flight speed (Jordano et al. 
2007). Specifically, for each simulated interaction event, the gut passage time and a flight speed were drawn from probability distributions based on allometric equations. Gut passage time and flight speed were multiplied yielding a dispersal distance which was corrected for time spent perching or non-directional flying (for detailed information on allometric equations see Sorensen et al. 2020, Donoso et al. 2020). Simulated seed-dispersal distances from this model are consistent with those reported from the few empirical studies available (Jordano et al. 2007, Kays et al. 2011).

We defined the 95th and the 99th percentile of the resulting 100 dispersal distances as two alternative proxies of the long-distance dispersal (LDD) ability of a plant species: 95\% LDD and 99\% LDD (Fig. 1 b). We focus on the 95\% LDD ability in accordance with recent modelling studies (e.g., Pires et al. 2018) and present results for $99 \%$ LDD in the supplement. We computed the LDD ability separately for each of the ten independent iterations. For further analyses, we considered the mean LDD ability across the ten iterations for each plant species. The variation among the ten runs was generally low (average coefficient of variation 0.21 ).

\section{Projected vertical temperature shifts}

We compared the simulated LDD ability of the plant species to projected vertical temperature shifts until 2070. For this purpose, we computed the average distances by which the plant species' current realized temperature range along the Manú gradient is projected to shift vertically. To do so, we computed the vertical temperature shift for each plant species based on projected anomalies in mean annual temperature (MAT) and the tropospheric lapse rate along the Manú gradient (Nowak et al. 2019; Fig. 1 c). We downloaded current and projected future MAT from WorldClim (Hijmans et al. 2005). WorldClim provides spatial interpolations of observed average climate conditions over the period 1960-1990. Data on future MAT are based on the IPCC's fifth assessment report (IPCC 2014). We used projected MAT for the year 2070 (averaged over 2061-2080) according to five general circulation models (CCSM4, HadGEM2-ES, MIROC 5, MRI-CGCM and NorESM1-M) and three representative concentration pathways (RCP) 2.6, 4.5, and 8.5. These RCPs represent a strong mitigation (RCP2.6), a moderate mitigation (RCP4.5), and a high emissions scenario (RCP8.5) (IPCC 2014). We took mean annual tropospheric lapse-rate values from Mokhov \& Akperov (2006). MAT and tropospheric lapse rate were analysed at a resolution of $2.5 \mathrm{~min}$. To estimate the projected vertical temperature shift for a plant 
species under a specific future scenario, we first calculated anomalies in MAT as the difference between the current and the projected future MAT for each grid cell intersected by the Manú gradient (i.e. nine grid cells, 2.5 min resolution, Tables S3, $\mathrm{S} 4)$. Second, we calculated the vertical temperature shift $(\mathrm{m})$ for each grid cell as temperature anomaly $\left({ }^{\circ} \mathrm{C}\right) /$ tropospheric lapse rate $\left({ }^{\circ} \mathrm{C} / \mathrm{m}\right)$. Third, we calculated the vertical temperature shift of a plant species as the mean vertical temperature shift across the grid cells intersected by the current elevational range (minimum elevation to maximum elevation) of the plant species along the Manú gradient (Nowak et al. 2019). The projected vertical temperature shift of a plant species under each of the three RCP scenarios was computed for each of the five general circulation models (GCM), resulting in 15 values of projected vertical temperature shift per species (Table S1).

\section{Required horizontal dispersal distances}

We estimated the horizontal dispersal distances required to track the projected vertical temperature shifts (Fig. $1 \mathrm{c}$ ), based on the average slope of the Manú gradient. To this end, we downloaded mean slope values globally at a $5 \mathrm{~km}$ spatial resolution (approximately $2.5 \mathrm{~min}$ ) from Amatulli et al. (2018). We computed the mean slope across six grid cells that capture the elevational range between 1000 to $3500 \mathrm{~m}$ a.s.I. along the Manú gradient (mean slope $11.45^{\circ} \pm 3.37 \mathrm{sd}$ ). We excluded the lowlands from this computation because we were interested in movements along the mountain slope and wanted to avoid an overestimation of the required dispersal distances for this mountain ecosystem. We estimated the required horizontal dispersal distances of a plant species for each RCP and GCM as the vertical temperature shift (m) divided by the sine of the mean slope $\left({ }^{\circ}\right)$.

\section{Projected number of LDD events required by 2070}

To estimate to what extent the LDD ability of a plant species may be sufficient to track projected vertical temperature shifts until 2070, we quantified for each plant species the projected number of LDD events required by 2070 under a given RCP and GCM (Fig. 1 d). This metric equals the number of LDD events a plant species would require to track projected vertical shifts of their current realized temperature range along the Manú gradient by 2070. We computed the projected number of LDD events required 
by 2070 by dividing the required horizontal dispersal distance $(\mathrm{m})$ of a plant species under a given RCP and GCM by the simulated LDD ability $(m)$ for each plant species.

\section{Results}

\section{Simulated dispersal ability and projected temperature shift}

Long-distance dispersal (LDD) ability varied among woody plant species and depended on the degree of trait matching (Fig. 2). For instance, it ranged from 48 to $702 \mathrm{~m}$ across plants species (193 $\pm 151 \mathrm{~m}$; mean $\pm \mathrm{sd} ; n=343$ plant species) in simulations with an intermediate degree of trait matching $(s=1.5)$, based on 95\% LDD ability. The LDD ability was largest in simulations with a low degree of trait matching (s $=0.5$ ). We found qualitatively similar results based on the 99\% LDD. In this case, the LDD ability was on average 1.5 to 1.7 times larger compared to 95\% LDD.

The projected vertical temperature shift until 2070 increased with the magnitude of warming (Table S1). Under RCP 2.6 (strong mitigation scenario, projected temperature anomaly for the region relative to $1960-1990$ : range $+1.29-1.83^{\circ} \mathrm{C}$ ), the average projected vertical temperature shift was $248 \pm 2 \mathrm{~m}$ (mean $\pm \mathrm{sd}, n=343$ plant species). In comparison, under RCP 4.5 (moderate mitigation scenario, range +1.9 $2.78^{\circ} \mathrm{C}$ ), it approximated $367 \pm 2 \mathrm{~m}$, and under RCP 8.5 (high emission scenario, range $+3.06-4.26^{\circ} \mathrm{C}$ ), it was estimated to be $566 \pm 3 \mathrm{~m}$. The corresponding required horizontal dispersal distances until 2070 were approximately fivefold larger (RCP 2.6, $1250 \pm 193 \mathrm{~m}$; RCP 4.5, $1846 \pm 243 \mathrm{~m}$; RCP 8.5, $2851 \pm 340 \mathrm{~m}$ ).

For the large majority of plant species, the projected number of LDD events required by 2070 was $>1$, even under scenarios of strong and moderate mitigation of greenhouse gas emissions (RCP 2.6, RCP 4.5; Fig. 3). Hence, the majority of plant species would require several successful LDD events before 2070 to fully track increasing temperatures along the elevational gradient. 


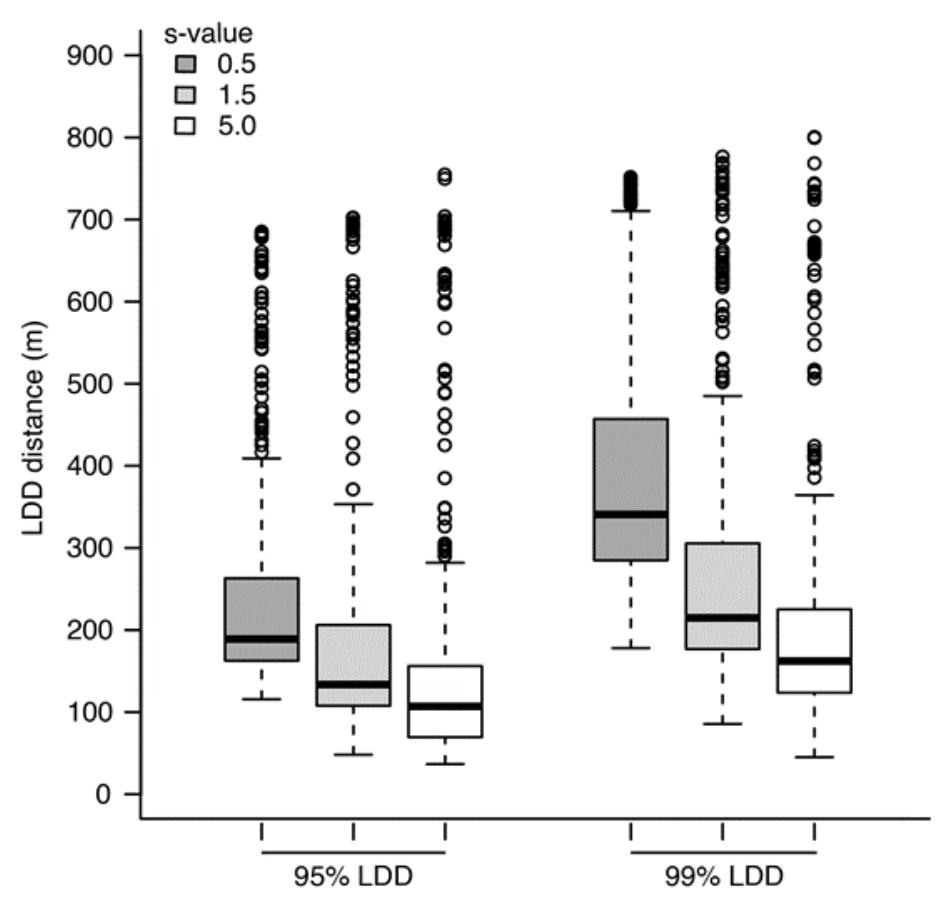

Figure 2: Trait-based estimates of long-distance dispersal (LDD) ability for 343 birddispersed woody plant species. (a) The LDD ability of the plant species has been estimated based on three trait-matching parameter values ( $s=5.0,1.5$, and 0.5 ), representing a range from a high to a low degree of trait matching (Fig. 1 a), and by two percentiles of the distribution of modelled seed dispersal distances for each plant species (95\% LDD, 99\% LDD; Fig. 1). Boxes indicate the median (line), 25th and 75th percentiles across plant species. Whiskers indicate the data range, and circles denote outliers.

\section{Degree of trait matching}

Consistent with our first hypothesis, the projected number of LDD events required by 2070 increased substantially with increasing degree of trait matching (Fig. 3a-c). For example, under a projected regional temperature anomaly of $+1.29^{\circ} \mathrm{C}$ (low end of RCP 2.6), the mean projected number of LDD events required by 2070 was 5.10 ( \pm 1.89 ; mean $\pm \mathrm{sd}, n=343$ plant species) in simulations based on a low degree of trait matching ( $s=0.5$; Fig. $3 \mathrm{a}$ ). In comparison, in simulations assuming a high degree of trait matching ( $s=5.0$; Fig. $3 \mathrm{c}$ ), the mean projected number of LDD events required by 2070 increased to10.82 ( \pm 6.28 ). The potential ability of plants to track temperature change decreased strongly in RCP scenarios with fewer mitigation measures. For instance, under a projected temperature anomaly of $+2.78^{\circ} \mathrm{C}$ (high end of RCP 4.5 ), the mean projected number of LDD events required by 2070 was estimated to be 11.06 $( \pm 4.01)$ in simulations assuming a low degree of trait matching (Fig. 3 a) and 23.44 $( \pm 13.47)$ in simulations assuming a high degree of trait matching (Fig. $3 \mathrm{c}$ ). Interestingly, the projected number of LDD events required by 2070 varied less among 
species in simulations with a low degree of trait matching. In simulations based on the 99\% LDD ability, qualitatively similar differences were found among simulations assuming different degrees of trait matching (Fig. S2).
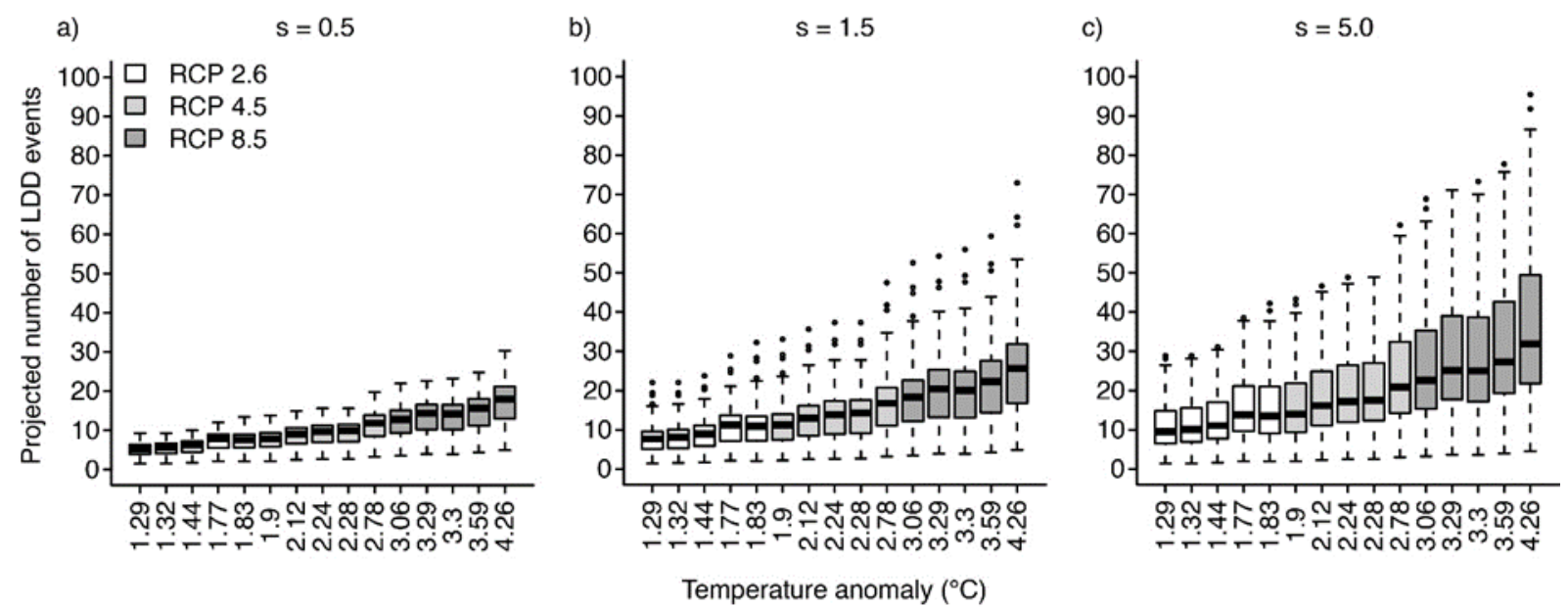

Figure 3: Projected number of long-distance dispersal (LDD) events required to track temperature change by 2070 for 343 bird-dispersed woody plant species. The number of LDD events equals the required horizontal dispersal distance divided by the LDD ability of that plant species. The LDD ability was estimated based on a (a) low, (b) intermediate and (c) high degree of trait matching ( $s=0.5,1.5$ and 5.0, respectively) and $95 \%$ LDD. Shown on the x-axis is the average projected anomaly in mean annual temperature until 2070 across the Manú gradient for each combination of emission scenario (RCP 2.6, 4.5 and 8.5) and climate model (five GCMs). Boxes indicate the median (line), 25th and 75th percentiles across plant species. Whiskers indicate the data range, and circles denote outliers.

\section{Plant traits}

Consistent with our second hypothesis, the projected number of LDD events required by 2070 was lower for large-fruited than for small-fruited plant species (Fig. 4 a, b). For instance, under RCP $2.6\left(+1.29^{\circ} \mathrm{C}\right)$, the mean projected number of LDD events for large-fruited species was $3.38( \pm 1.74$; mean $\pm \mathrm{sd}, n=86$ plant species $)$ in simulations assuming an intermediate degree of trait matching ( $s=1.5$; Fig. 4 a). In comparison, the mean projected number of LDD events for small-fruited species was $8.91( \pm 2.76$; Fig. 4 b). Under RCP $4.5\left(+2.78^{\circ} \mathrm{C}\right)$, the mean projected number of LDD events was 7.34 ( \pm 3.69; Fig. 4 a) for large-fruited species and 19.25 ( \pm 5.66 ) for small-fruited species (Fig. 4b).

Similarly, the projected number of LDD events was lower for canopy than for understory plant species (Fig $4 \mathrm{c}, \mathrm{d}$ ). However, this difference was less pronounced than the difference between large-and small-fruited plant species. Under RCP 2.6 (+ $\left.1.29^{\circ} \mathrm{C}\right)$, the mean projected number of LDD events required by 2070 was $5.70( \pm 3.38$; 
Fig. 4 c) for canopy species and $8.66( \pm 2.35$; Fig. 4 d) for understory species. Under RCP $4.5\left(+2.78^{\circ} \mathrm{C}\right)$, the mean projected number of LDD events was $12.41( \pm 7.22)$ for canopy species and 18.84 ( \pm 4.94) for understory species. Qualitatively similar differences between plant species with different traits were found for different degrees of trait matching (Fig. S3, S4) and 99\% LDD ability (Table S1).
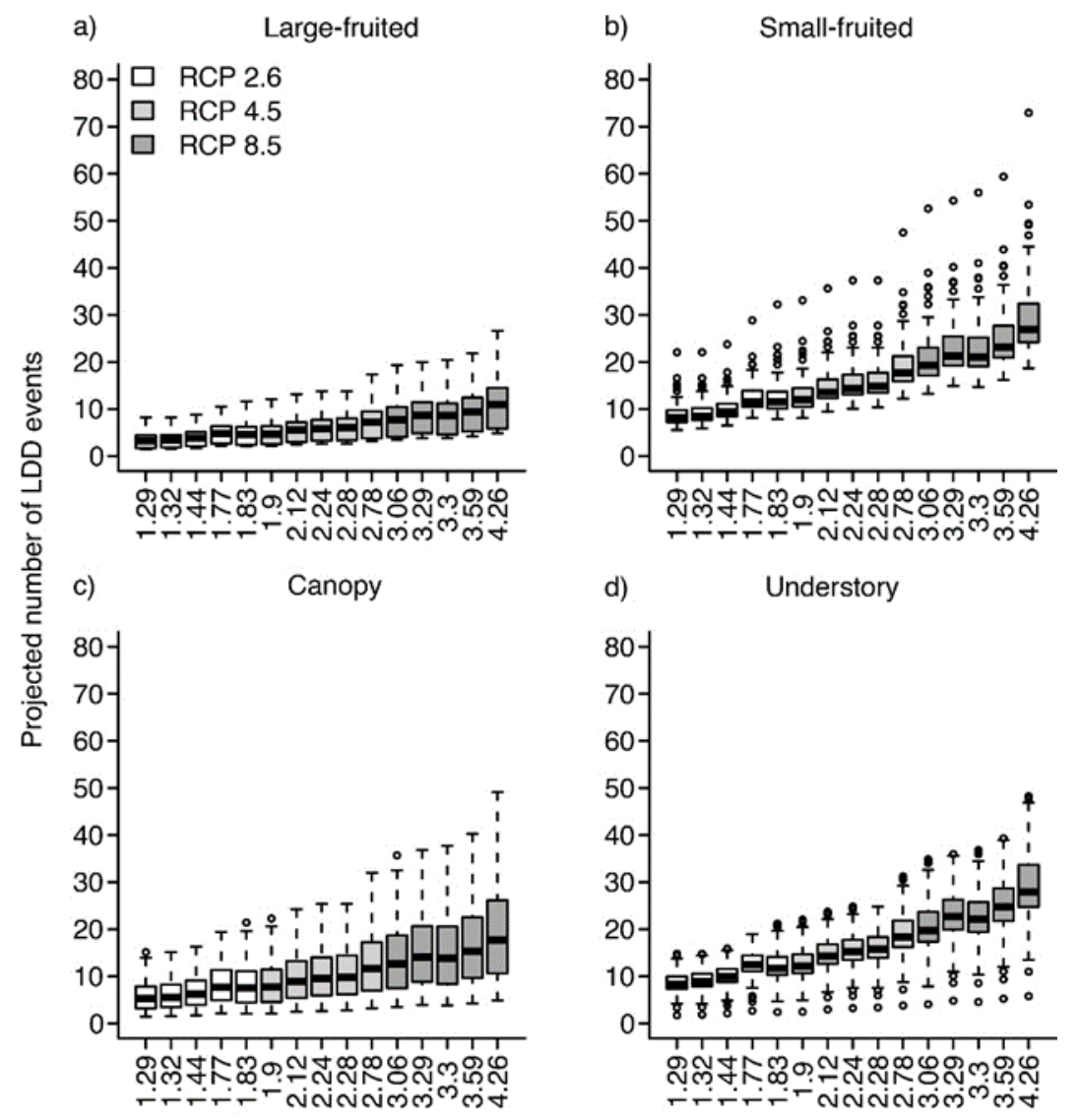

Temperature anomaly $\left({ }^{\circ} \mathrm{C}\right)$

Figure 4: Projected number of long-distance dispersal (LDD) events required to track temperature change by 2070 for (a) large-fruited vs. (b) small-fruited and (c) canopy vs. (d) understory bird-dispersed woody plant species ( $n=86$, respectively). The number of required LDD events equals the required horizontal dispersal distance divided by the LDD ability of that plant species. LDD ability was estimated based on an intermediate degree of trait matching $(s=1.5)$ and 95\% LDD. Shown on the $x$-axis is the average projected anomaly in mean annual temperature until 2070 across the Manú gradient for each combination of emission scenario (RCP 2.6, 4.5 and 8.5) and climate model (five GCMs). Boxes indicate the median (line), 25th and 75th percentiles across plant species. Whiskers indicate the data range, and circles denote outliers.

\section{Discussion}

With a new combination of trait-based approaches, we quantified the ability of birddispersed woody plant species to track projected vertical temperature shifts until 2070 on a tropical mountain. Our simulations suggest that the LDD ability of many plant 
species may be insufficient, as plants were projected to require several LDD events to track vertical temperature shifts by 2070 , even under scenarios of strong and moderate mitigation of greenhouse gas emissions. Previous studies have shown that seed dispersal by mammals can help plant species to track temperature shifts on mountains (Naoe et al. 2016, González-Varo et al. 2017). In contrast, our simulations suggest that avian-mediated seed dispersal may be insufficient for plants to track their temperature niche on mountains. Our results are in line with the discrepancy between observed vertical range shifts of tropical tree genera and vertical temperature shifts on tropical mountains (Feeley et al. 2011). Importantly, our simulations further show that both the degree of trait matching and plant traits influence to what extent plant species may lag behind climate change.

Our results imply that plants in seed-dispersal systems with a low degree of trait matching may be more resilient to climate change because they are better able to track temperature changes via dispersal. This is because large-billed and large-bodied frugivores feed on a wider range of plant species in such systems which increases the diversity of seed dispersers for small-fruited species and the probability that their seeds get dispersed over long distances (Sorensen et al. 2020). The similarity in seed dispersers across plant species also explains why differences among plants' dispersal ability are less pronounced under low compared to high trait matching scenarios (Sorensen et al. 2020). The effect of trait matching on seed dispersal has important implications for understanding how the response of animal-dispersed plants to climate change varies among communities. It has been found that seed-dispersal networks are less specialized at low than at high latitudes (Dalsgaard et al. 2017) and in the Afrotropics compared to the Neotropics (Dugger et al. 2019). Furthermore, the specialization of seed-dispersal networks can increase with elevation (Quitián et al. 2018) and is larger in the forest interior than at forest edges (Menke et al. 2012). This variability of specialization in seed-dispersal systems suggests that the ability of birddispersed plants to track climate change may vary substantially both globally and locally.

A low degree of specialization is generally considered beneficial for species facing environmental change (Gallagher et al. 2015) and earlier work quantifying species' susceptibility to climate change have focussed on species' habitat or diet specialization (MacLean and Beissinger 2017). In contrast, the effects of biotic specialization in complex interaction networks have been less thoroughly explored 
(Schleuning et al. 2016, Bascompte et al. 2019). Previous work suggests that biotic interactions with a low degree of specialization are functionally more redundant and more robust against extinction cascades resulting from the loss of interaction partners (Brodie et al. 2014). Our results imply that the specialization of seed-dispersal networks is not only important for mediating the risk of secondary extinctions, but may also directly influence the ability of plants to track climate change.

We further found a greater ability of large-fruited and canopy plant species to track projected temperature shifts compared to small-fruited and understory species. This effect is driven by the higher preference of large-billed and large-sized frugivores for large-fruited plant species that tend to be more abundant in the forest canopy (Shanahan and Compton 2001). Importantly, we found this effect in simulations with different degrees of trait matching. In line with our finding, fossil pollen data indicate that large-seeded plant assemblages have tracked rapid climate change more closely than small-seeded plant assemblages in the Late Quaternary (Knight et al. 2020). Similarly, large-seeded understory plants have been observed to better track contemporary climate change than small-seeded ones (Ash et al. 2017). In addition to fruit size, plant height has also been shown to be positively associated with dispersal distance within and across dispersal modes (Thomson et al. 2011). Importantly, fruit size and seed size, as well as plant height, also impact other aspects of plants' demography and colonization potential. While fruit removal tends to be higher for smallseeded canopy plant species (Schleuning et al. 2011, Muñoz et al. 2017), seedling survival and recruitment probability are usually higher for large-seeded species and understory species (Moles and Westoby 2004, Muñoz et al. 2017). Nevertheless, plant species with different traits usually achieve similar lifetime fitness because traits that promote a high dispersal ability usually relate to lower recruitment success, and vice versa (Terborgh et al. 2014). Our findings suggest that the checks and balances in such life-history trade-offs may potentially be shifted towards large-seeded canopy species as these species have a higher ability to track changing temperatures in a quickly changing environment. Further studies are required to test to what extent such indirect, animal-mediated effects on plant traits trigger changes in plant evolution and community structure under climate change (Sales et al. 2021).

Importantly, the dispersal ability of plant species in response to climate warming may strongly interact with other drivers of global change. Defaunation and the subsequent downsizing of animal communities have been shown to severely affect 
large-fruited plant species (Dirzo et al. 2014). Specifically, the downsizing of frugivore communities can substantially reduce the LDD ability of bird-dispersed plants even if only a few large dispersers are lost (Donoso et al. 2020). Although large-fruited canopy plant species might be more likely to keep up with rapid climate change, this implies that they might quickly lose dispersal ability in defaunated ecosystems and that the conservation of functionally diverse communities of avian seed dispersers will be pivotal to promote tracking of climate shifts by plants. Defaunation effects on seed dispersal could be further accelerated by observed declines in disperser body size as a direct response to climate change (Weeks et al. 2020) and by projected losses of large frugivores under climate change (Sales et al. 2021).

By applying a new combination of trait-based models, we shed light on how the degree of trait matching and plant traits influence the ability of bird-dispersed plants to track projected temperature shifts over the coming decades. Such knowledge is highly relevant in light of rapid climate change as it can increase our understanding of which species may be able to track climate changes and which species require assisted migration (Lenoir et al. 2020). Nevertheless, we acknowledge that our trait-based simulation approach has limitations. Our seed-dispersal model builds on a rather small empirical data source and validation with more empirical seed-dispersal data would be desirable (Sorensen et al. 2020). A comparison of our simulation results to observed plant range shifts on tropical mountains (Feeley et al. 2011, Morueta-Holme et al. 2015) could be enlightening in future work. Moreover, our model is limited to bird-dispersed plants, but other animal vectors of seed dispersal, such as mammals, may provide longer dispersal distances and play a prominent role for plants to escape warming (Naoe et al. 2016, González-Varo et al. 2017). Finally, our projections consider a fixed time window until 2070 and do not account for differences in plant species' generation times. For the majority of plant species studied here, it is unlikely that they could undergo multiple LDD events by 2070 because woody plant species have long generation times which slows down their ability to track climate change (SalgueroGómez et al. 2015).

Our study demonstrates a new way of using functional traits to project the ability of bird-dispersed plants to track temperature change. In a tropical mountain system, we find that avian seed dispersal appears to be insufficient for the large majority of woody plant species to track vertical temperature shifts over the next decades. Our study proposes a relatively simple trait-based tool to approximate the dispersal ability 
of bird-dispersed plant species and only requires information on plant-frugivore cooccurrences and functional plant and bird traits. The increasing availability of functional trait data of plants and birds globally will allow parameterizing similar models for both lateral and vertical seed dispersal for many other types of avian seed-dispersal systems. Such trait-based dispersal models could prospectively be integrated into projection models of biodiversity in a rapidly changing world.

\section{References}

Amatulli, G. et al. 2018. Data Descriptor: A suite of global, cross-scale topographic variables for environmental and biodiversity modeling. - Sci. Data 5: 1-15.

Ash, J. D. et al. 2017. Tracking lags in historical plant species' shifts in relation to regional climate change. - Glob. Chang. Biol. 23: 1305-1315.

Bascompte, J. et al. 2019. Mutualistic interactions reshuffle the effects of climate change on plants across the tree of life. - Sci. Adv. 5: eaav2539.

Bender, I. M. A. et al. 2017. Functionally specialised birds respond flexibly to seasonal changes in fruit availability. - J. Anim. Ecol. 86: 800-811.

Bender, I. M. A. et al. 2018. Morphological trait matching shapes plant-frugivore networks across the Andes. - Ecography 41: 1-10.

Brako, L. and Zarucchi, J. L. 1993. Catalogue of the flowering plants and Gymnosperms of Peru. Catálogo de las Angiospermas y Gimnospermas del Perú.

Brodie, J. F. et al. 2014. Secondary extinctions of biodiversity. - Trends Ecol. Evol. 29: 664-672.

Brommer, J. E. et al. 2012. The breeding ranges of Central European and Arctic bird species move poleward. - PLoS One 7: e43648.

Burns, K. C. 2013. What causes size coupling in fruit-frugivore interaction webs? Ecology 94: 295-300.

Cain, M. L. et al. 2000. Long-distance seed dispersal in plant populations. - Am. J. Bot. 87: 1217-1227.

Chen, S. C. et al. 2017. Plants show more flesh in the tropics: variation in fruit type along latitudinal and climatic gradients. - Ecography 40: 531-538.

Chen, S. et al. 2019. Seeds tend to disperse further in the tropics. - Ecol. Lett. 22: 954961. 
Dalsgaard, B. et al. 2017. Opposed latitudinal patterns of network-derived and dietary specialization in avian plant-frugivore interaction systems. - Ecography 40: 13951401.

Dehling, D. M. et al. 2013. Upper and lower elevational extremes of Andean birds from south-east Peru. - Boletín Inf. 8: 32-38.

Dehling, D. M. et al. 2014. Functional relationships beyond species richness patterns: trait matching in plant-bird mutualisms across scales. - Glob. Ecol. Biogeogr. 23: 1085-1093.

Dirzo, R. et al. 2014. Defaunation in the Anthropocene. - Science 345: 401-406.

Donoso, I. et al. 2017. Defaunation effects on plant recruitment depend on size matching and size trade-offs in seed-dispersal networks. - Proc. R. Soc. B Biol. Sci. 284: 20162664.

Donoso, I. et al. 2020. Downsizing of animal communities triggers stronger functional than structural decay in seed-dispersal networks. - Nat. Commun. 11: 1-8.

Dugger, P. J. et al. 2019. Seed-dispersal networks are more specialized in the Neotropics than in the Afrotropics. - Glob. Ecol. Biogeogr. 28: 248-261.

Dunning, J. B. 2007. CRC Handbook of Avian Body Masses. - CRC Press.

Eck, S. et al. 2011. Measuring birds. - Christ Media Natur.

Estrada, A. et al. 2015. Species' intrinsic traits inform their range limitations and vulnerability under environmental change. - Glob. Ecol. Biogeogr. 24: 849-858.

Feeley, K. J. et al. 2011. Upslope migration of Andean trees. - J. Biogeogr. 38: 783791.

Fründ, J. et al. 2016. Sampling bias is a challenge for quantifying specialization and network structure: lessons from a quantitative niche model. - Oikos 125: 502513.

Gallagher, A. J. et al. 2015. Evolutionary theory as a tool for predicting extinction risk. - Trends Ecol. Evol. 30: 61-65.

Girardin, C. A. J. et al. 2010. Net primary productivity allocation and cycling of carbon along a tropical forest elevational transect in the Peruvian Andes. - Glob. Chang. Biol. 16: 3176-3192.

Girardin, C. A. J. et al. 2013. Fine root dynamics along an elevational gradient in tropical Amazonian and Andean forests. - Global Biogeochem. Cycles 27: 252264. 
González-Varo, J. P. et al. 2017. Seed dispersers help plants to escape global warming. - Oikos 126: 1600-1606.

Hampe, A. 2011. Plants on the move: the role of seed dispersal and initial population establishment for climate-driven range expansions. - Acta Oecologica 37: 666673.

Hijmans, R. J. et al. 2005. Very high resolution interpolated climate surfaces for global land areas. - Int. J. Climatol. 25: 1965-1978.

IPCC 2014. Climate change 2014: synthesis report. Contribution of working groups I, II and III to the fifth assessment report of the Intergovernmental Panel on Climate Change [Core Writing Team, R.K. Pachauri and L.A. Meyer (eds.)].

Jordano, P. 2014. Fruits and frugivory. - In: Seeds: the ecology of regeneration in plant communities. 3rd ed.n. pp. 18-61.

Jordano, P. et al. 2007. Differential contribution of frugivores to complex seed dispersal patterns. - Proc. Natl. Acad. Sci. 104: 3278-3282.

Kays, R. et al. 2011. The effect of feeding time on dispersal of Virola seeds by toucans determined from GPS tracking and accelerometers. - Acta Oecologica 37: 625631.

Knight, C. A. et al. 2020. Community assembly and climate mismatch in Late Quaternary eastern North American pollen assemblages. - Am. Nat. 195: 166180.

Lenoir, J. et al. 2020. Species better track climate warming in the oceans than on land. - Nat. Ecol. Evol. 4: 1044-1059.

MacLean, S. A. and Beissinger, S. R. 2017. Species' traits as predictors of range shifts under contemporary climate change: a review and meta-analysis. - Glob. Chang. Biol. 23: 4094-4105.

Menke, S. et al. 2012. Plant-frugivore networks are less specialized and more robust at forest-farmland edges than in the interior of a tropical forest. - Oikos 121: 15531566.

Mokhov, I. I. and Akperov, M. G. 2006. Tropospheric lapse rate and its relation to surface temperature from reanalysis data. - Izv. Atmos. Ocean. Phys. 42: 430438.

Moles, A. T. and Westoby, M. 2004. Seedling survival and seed size: a synthesis of the literature. - J. Ecol. 92: 372-383.

Moles, A. T. et al. 2009. Global patterns in plant height. - J. Ecol. 97: 923-932. 
Morueta-Holme, N. et al. 2015. Strong upslope shifts in Chimborazo's vegetation over two centuries since Humboldt. - Proc. Natl. Acad. Sci. 112: 12741-12745.

Muñoz, M. C. et al. 2017. Importance of animal and plant traits for fruit removal and seedling recruitment in a tropical forest. - Oikos 126: 823-832.

Naoe, S. et al. 2016. Mountain-climbing bears protect cherry species from global warming through vertical seed dispersal. - Curr. Biol. 26: R315-R316.

Nowak, L. et al. 2019. Projecting consequences of global warming for the functional diversity of fleshy-fruited plants and frugivorous birds along a tropical elevational gradient. - Divers. Distrib. 25: 1362-1374.

Patterson, B. D. et al. 1998. Contrasting patterns of elevational zonation for birds and mammals in the Andes of southeastern Peru. - J. Biogeogr. 25: 593-607.

Pecl, G. T. et al. 2017. Biodiversity redistribution under climate change: impacts on ecosystems and human well-being. - Science 355: eaai9214.

Pires, M. M. et al. 2018. Pleistocene megafaunal extinctions and the functional loss of long-distance seed-dispersal services. - Ecography 41: 153-163.

Quitián, M. et al. 2018. Elevation-dependent effects of forest fragmentation on plantbird interaction networks in the tropical Andes. - Ecography 41: 1497-1506.

Quitián, M. et al. 2019. Direct and indirect effects of plant and frugivore diversity on structural and functional components of fruit removal by birds. - Oecologia 189: 435-445.

Sales, L. P. et al. 2021. Climate change reshapes the eco-evolutionary dynamics of a Neotropical seed dispersal system. - Glob. Ecol. Biogeogr. 00: 1-10.

Salguero-Gómez, R. et al. 2015. The COMPADRE Plant Matrix Database: an open online repository for plant demography. - J. Ecol. 103: 202-218.

Schleuning, M. et al. 2011. Specialization and interaction strength in a tropical plantfrugivore network differ among forest strata. - Ecology 92: 26-36.

Schleuning, M. et al. 2016. Ecological networks are more sensitive to plant than to animal extinction under climate change. - Nat. Commun. 7: 13965.

Schleuning, M. et al. 2020. Trait-Based assessments of climate-change impacts on interacting species. - Trends Ecol. Evol. 35: 319-328.

Shanahan, M. and Compton, S. G. 2001. Vertical stratification of figs and fig-eaters in a Bornean lowland rain forest: how is the canopy different? - Plant Ecol. 153: $121-132$. 
Sorensen, M. C. et al. 2020. Community-wide seed dispersal distances peak at low levels of specialisation in size-structured networks. - Oikos 129: 1727-1738.

Terborgh, J. et al. 2014. How many seeds does it take to make a sapling? - Ecology 95: 991-999.

Thomson, F. J. et al. 2011. Seed dispersal distance is more strongly correlated with plant height than with seed mass. - J. Ecol. 99: 1299-1307.

Tropicos 2017. Tropicos. - Missouri Bot. Gard.

Walker, B. et al. 2006. Birds of the Manu Biosphere Reserve. - Fieldiana Zool.: 23-49.

Weeks, B. C. et al. 2020. Shared morphological consequences of global warming in North American migratory birds. - Ecol. Lett. 23: 316-325.

Westcott, D. A. and Graham, D. L. 2000. Patterns of movement and seed dispersal of a tropical frugivore. - Oecologia 122: 249-257.

Wheelwright, N. T. 1985. Fruit-size, gape width, and the diets of fruit-eating birds. Ecology 66: 808-818.

\section{Data accessibility}

The data supporting the results has been uploaded as part of the electronic supplementary information. We confirm that, should the manuscript be accepted, the data will be archived at Dryad. 


\section{Supporting information}

\section{Figures}

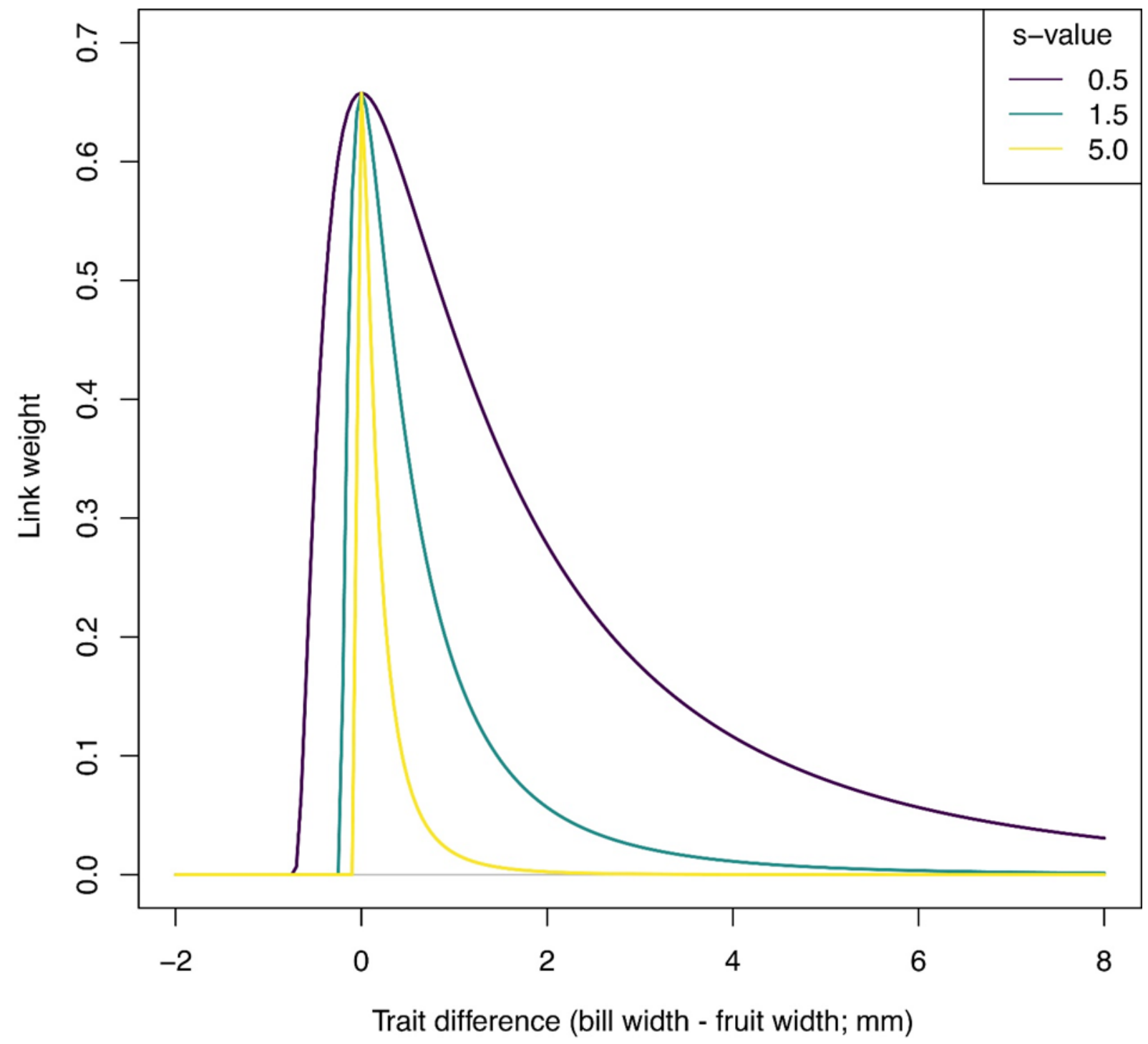

Figure S1: Relationship of link weight and trait difference for three trait-matching parameter values $(s=0.5,1.5,5.0)$ representing a low, an intermediate and a high degree of trait matching. The trait difference has been computed as bill width minus fruit width, therefore negative values indicate that the fruit is larger than the bill, while positive values indicate that the bill is larger than the fruit. The link weights have been computed as dlnorm $\left(x^{*} s+e^{\wedge}-1\right)$ where $x=$ trait difference $(\mathrm{mm})$ and $s=$ trait-matching parameter value. The dlnorm-function was chosen to represent a skewed niche shape that accounts for forbidden links if the fruit is larger than the bill. For the analysis, the link weights are transformed to interaction probabilities by standardizing them by the number of links per bird species which is the default in the model proposed by Donoso et al. (2017). 

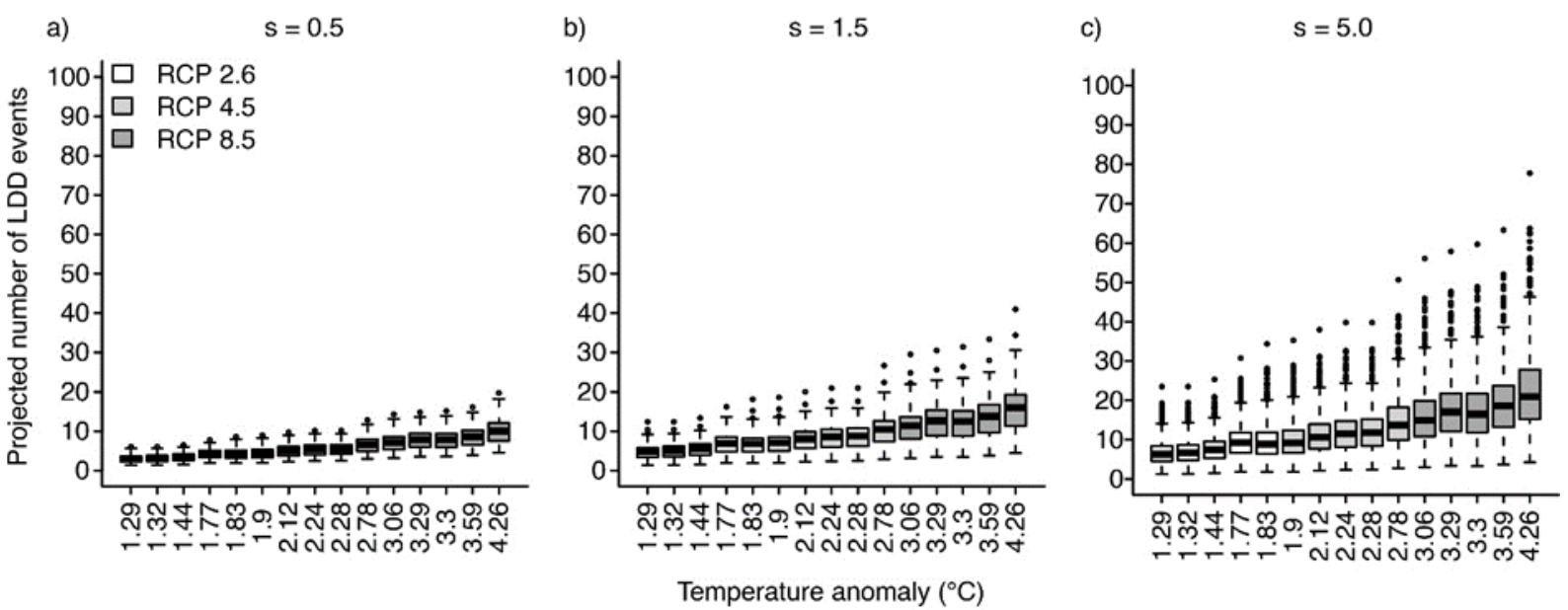

Figure S2: Projected number of long-distance dispersal (LDD) events required to track temperature change by 2070 for 343 bird-dispersed woody plant species and $99 \%$ LDD. The number of LDD events equals the required horizontal dispersal distance divided by the LDD ability of a plant species. The LDD ability of the plant species has been estimated based on a (a) low, (b) intermediate and (c) high degree of trait matching ( $s=0.5,1.5$ and 5.0 , respectively). Shown on the $x$-axis is the average projected anomaly in mean annual temperature until 2070 across the Manú-gradient for each combination of emission scenario (RCP 2.6, 4.5 and 8.5) and climate model (five GCMs). Boxes indicate the median (line), 25th and 75th percentiles across plant species. Whiskers indicate the data range, and circles denote outliers. 


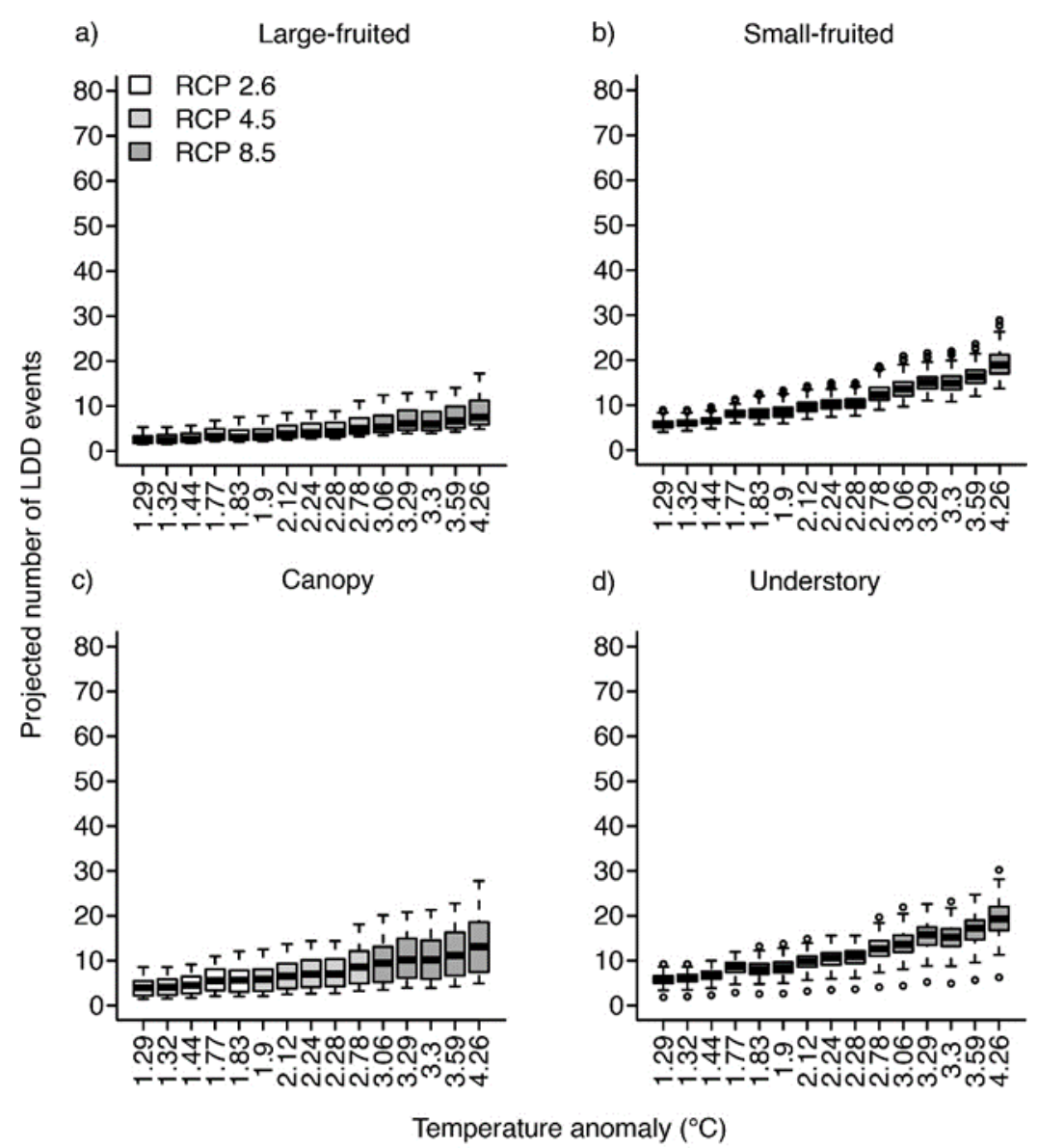

Figure S3: Projected number of long-distance dispersal (LDD) events required to track temperature change by 2070 for (a) large-fruited vs. (b) small-fruited and (c) canopy vs. (d) understory bird-dispersed woody plant species $(n=86$, respectively) considering a low degree of trait matching $(s=0.5)$ and the $95 \%$ LDD. The number of required LDD events equals the required horizontal dispersal distance divided by the LDD ability of a plant species. Shown on the $x$-axis is the average projected anomaly in mean annual temperature until 2070 across the Manú-gradient for each combination of emission scenario (RCP 2.6, 4.5 and 8.5) and climate model (five GCMs). Boxes indicate the median (line), 25th and 75th percentiles across plant species. Whiskers indicate the data range, and circles denote outliers. 


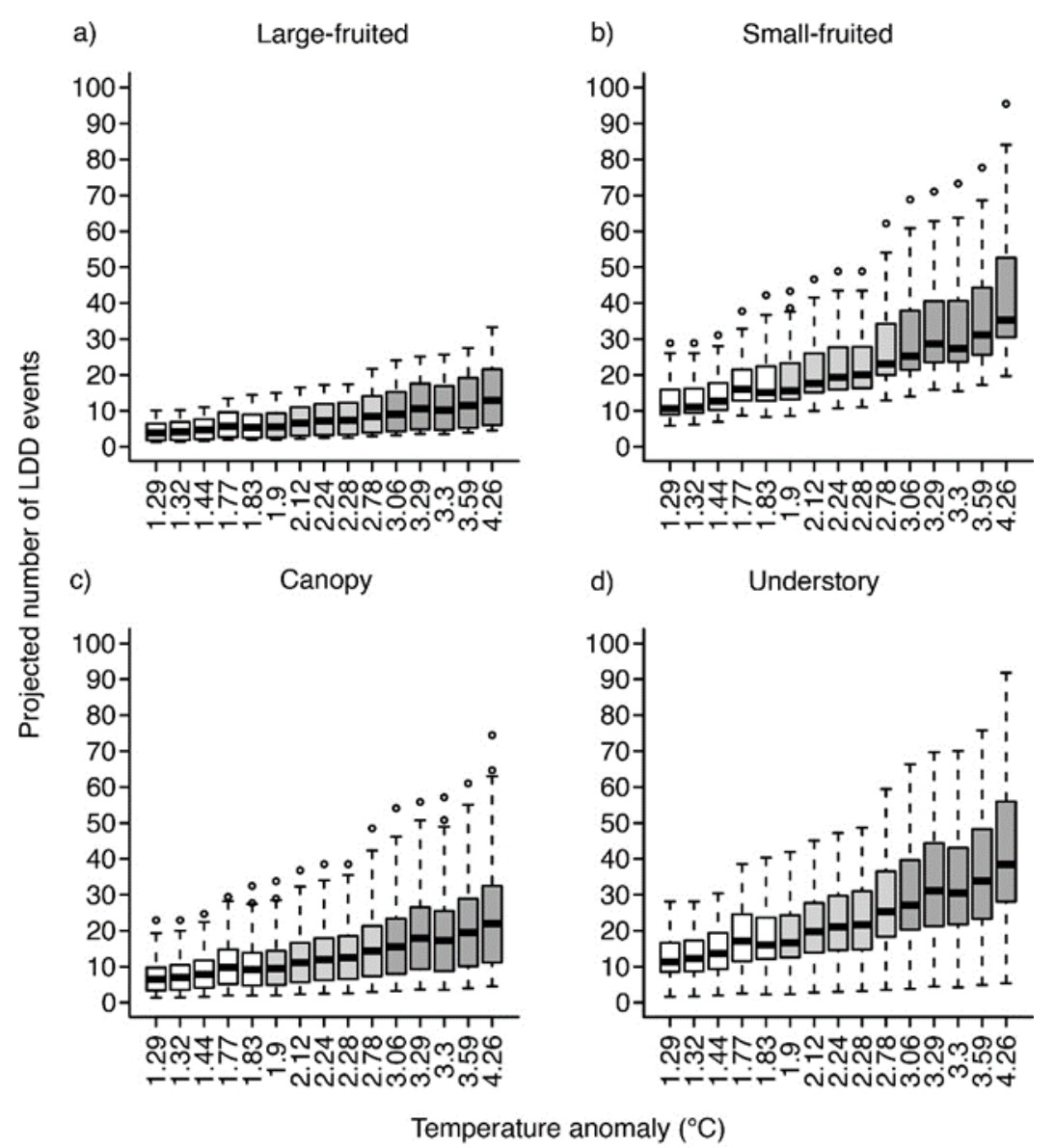

Figure S4: Projected number of long-distance dispersal (LDD) events required to track temperature change by 2070 for (a) large-fruited vs. (b) small-fruited and (c) canopy vs. (d) understory bird-dispersed woody plant species $(n=86$, respectively) considering a high degree of trait matching $(s=5.0)$ and the $95 \%$ LDD. The number of required LDD events equals the required horizontal dispersal distance divided by the LDD ability of a plant species. Shown on the $x$-axis is the average projected anomaly in mean annual temperature until 2070 across the Manú-gradient for each combination of emission scenario (RCP 2.6, 4.5 and 8.5) and climate model (five GCMs). Boxes indicate the median (line), 25th and 75th percentiles across plant species. Whiskers indicate the data range, and circles denote outliers.

\section{Tables}

Tables $\mathbf{S} 1$ and $\mathbf{S} 2$ are data files provided in a separate excel file. 
Table S3: Studied elevations at the Manú elevational gradient. Plant data were collected at 500-3500 m a. s. I. Vertical distances were projected considering plant species' occurrences within 250-3750 m a.s.I. The mean slope of the Manú gradient was computed across 1000 to $3500 \mathrm{~m}$ a.s.I.. Given is the mean slope per grid cell at a $5 \mathrm{~km}$ spatial resolution.

\begin{tabular}{llll}
\hline Elevation (m a.s.I.) & Longitude & Latitude & Mean slope $\left.\mathbf{(}^{\circ}\right)$ \\
\hline 250 & -70.60 & -12.48 & - \\
\hline 500 & -71.23 & -12.65 & - \\
\hline 1000 & -71.27 & -12.63 & 4.97 \\
\hline 1500 & -71.54 & -13.05 & 10.63 \\
\hline 2000 & -71.55 & -13.07 & 13.21 \\
\hline 2500 & -71.57 & -13.09 & 13.69 \\
\hline 3000 & -71.58 & -13.17 & 12.60 \\
\hline 3500 & -71.60 & -13.12 & 13.60 \\
\hline 3750 & -71.65 & -13.15 & - \\
\hline
\end{tabular}

Table S4: Average projected anomaly in mean annual temperature $\left({ }^{\circ} \mathrm{C}\right)$ for the year 2070 , three greenhouse gas emission scenarios (RCP) and five general circulation models (GMC). Given are mean and SD across the nine elevations that capture the Manú gradient.

\begin{tabular}{llll}
\hline Future scenario & GCM & Mean anomaly $\left({ }^{\circ} \mathbf{C}\right)$ & SD anomaly $\left({ }^{\circ} \mathbf{C}\right)$ \\
\hline RCP 2.6 & MRI-CGCM & 1.29 & 0.06 \\
\hline RCP 2.6 & NorESM1-M & 1.32 & 0.04 \\
\hline RCP 2.6 & CCSM4 & 1.44 & 0.07 \\
\hline RCP 2.6 & MIROC 5 & 1.77 & 0.11 \\
\hline RCP 2.6 & HadGEM2-ES & 1.83 & 0.09 \\
\hline RCP 4.5 & MRI-CGCM & 1.90 & 0.10 \\
\hline RCP 4.5 & NorESM1-M & 2.12 & 0.04 \\
\hline RCP 4.5 & CCSM4 & 2.24 & 0.07 \\
\hline RCP 4.5 & MIROC 5 & 2.28 & 0.11 \\
\hline RCP 4.5 & HadGEM2-ES & 2.78 & 0.04 \\
\hline RCP 8.5 & MRI-CGCM & 3.06 & 0.10 \\
\hline RCP 8.5 & MIROC 5 & 3.29 & 0.14 \\
\hline RCP 8.5 & NorESM1-M & 3.30 & 0.00 \\
\hline RCP 8.5 & CCSM4 & 3.59 & 0.14 \\
\hline RCP 8.5 & HadGEM2-ES & 4.26 & 0.09 \\
\hline
\end{tabular}

\section{References}

Donoso, I. et al. 2017. Defaunation effects on plant recruitment depend on size matching and size trade-offs in seed-dispersal networks. - Proc. R. Soc. B Biol. Sci. 284: 20162664. 


\section{A4: Curriculum Vitae}

\section{Larissa Nowak}

larissanowak89@gmail.com

Google Scholar

\section{Education}

Since 2016

\section{PhD in Ecology}

Senckenberg Biodiversity and Climate Research Centre,

Goethe University, Frankfurt am Main, Germany

Dissertation title: 'Impacts of climate change on interacting plant and bird species - A trait-based perspective'

Project includes:

- Assessing relationships of response traits related to species' sensitivity to climate change and their adaptive capacity

- Projecting consequences of climate change for the functional diversity of fleshy-fruited plants and frugivorous birds

- Trait-based quantifications of long-distance dispersal of fleshy-fruited plants under climate change

First supervisor: PD Dr Matthias Schleuning

Second supervisor: Dr W. Daniel Kissling

\section{M.Sc. Ecology and Evolution}

Goethe University, Frankfurt am Main, Germany

Date of graduation: 10 February 2016

Courses included:

- Community Ecology, Macroecology and Conservation

- Parasitology

- Molecular Evolution and Population Genetics

- Diversity and Evolution of Plants

- Mycology

Thesis title: 'Global patterns in the functional diversity of frugivorous birds in seed-dispersal networks'

Supervisor: PD Dr Matthias Schleuning 
B.Sc. Environmental Science and International Forestry

Albert-Ludwig University, Freiburg, Germany

Date of graduation: 12 February 2013

Courses included:

- Biology and Ecology

- Wildlife Ecology and Management

- Soil Science

- Material Cycles in Ecosystems

- Environmental Modelling

- Forest Ecology

- Fire Ecology and Management

- Forest Management

Thesis title: 'Sensitivitätsanalye: Der Einfluss der Waldkalkung auf das Grüne Besenmoos (Dicranum viride)'

(Sensitivity assessment: 'The influence of forest liming on

Dicranum viride')

Supervisor: Prof. Dr Albert Reif

2008

\section{University entrance qualification (Abitur)}

Kopernikus Gymnasium Wasseralfingen, Germany

Scientific positions

since $02 / 2021$

$11 / 2020-01 / 2021$

$10 / 2016-10 / 2020$
Research fellow in 'Modelling species invasions', Senckenberg Biodiversity and Climate Research Centre

Research fellow in Emmy Noether Programme, 'Macroevolution of climatic niches in birds', Senckenberg Biodiversity and Climate Research Centre

Research fellow in 'Impacts of climate change on interacting plant and bird species', Senckenberg Biodiversity and Climate Research Centre 
Scientific courses

2019

2018

2017

Research stays

2019

2017

2016

2016
Species Distribution Modelling

Scientific English

Network Analysis for Ecologists
Research stay in the Manú National Park, Peru

Visiting study system and research plots of my PhD project

Research stay at the University of Amsterdam, Netherlands Biogeography \& Macroecology (BIOMAC) lab of Dr W. Daniel Kissling

Research stay at the Zoological Research Museum Alexander Koenig in Bonn, Germany, collection of bird skins, curator Dr Till Töpfer, morphological measurements on museum bird specimens

Research stay at the Natural History Museum in Copenhagen, Denmark, collection of bird skins, curator Prof. Dr Jon Fjeldsa, morphological measurements on museum bird specimens

\section{Internships and further professional experience}

Since 2018

Assistance in the statistic course of the Master's module Community Ecology, Macroecology and Conservation, Goethe University and Senckenberg Biodiversity and Climate Research Centre, Frankfurt am Main, Germany

- ANOVA

- Generalized linear models

- Linear mixed effect models

- Multiple regression

- Multivariate statistics

Since 2018

Organization of monthly meetings of the Functional Ecology and Global Change lab at Senckenberg Biodiversity and Climate Research Centre, Frankfurt am Main, Germany 
2015 - $2016 \quad$ Research assistance at Senckenberg Biodiversity and

Climate Research Centre, Frankfurt am Main, Germany

- Assistance in the organization of the Master's module Community Ecology, Macroecology and Conservation

- Guiding students with the discussion of scientific publications

- Data inspection (hummingbird-Protea interactions)

- Literature review (European oaks)

- Data management (global database of plant-frugivore networks, plant and frugivore traits)

2012

Internship at Forstliche Versuchs- und Forschungsanstalt Baden-Württemberg (forestry research institute), section forest ecology, Freiburg, Germany

- Assistance in Natura 2000 interests (FFH-Management plans, Red List species)

- Supervision of information stand 'Alt- und Totholzkonzept Baden-Württemberg' (Matured forest and deadwood concept) at KFW-Tagung Baden-Württemberg

- Attendance at workshop 'Waldnaturschutzstrategie' (Forest conservation strategy)

- Assistance in the section Forest Plant Breeding and Genetics

- Collaboration in scientific project on the sensitivity of Dicranum viride to forest liming

- Assistance in collecting movement data of wild cats and roe deer

- Assistance in bark beetle monitoring

\section{Language and communication skills}

German: native speaker

English: excellent command

Excellent written and oral scientific communication skills (German and English)

\section{R skills}

Excellent statistical and modelling skills with $R$

Selection of packages I have worked with:

ape, ade4, biomod2, bipartite, caper, ecospat, FD, ggplot2, hypervolume, Ime4, maps, MuMin, quantreg, raster, rgdal, $s p$

\section{Software skills}

Proficient with Windows, MS Office

Working knowledge in Mac OS and Adobe Illustrator 


\section{A5: Publications and scientific presentations}

\section{Publications}

Nowak, L., Kissling, W. D., Bender, I. M. A., Dehling, D. M., Töpfer, T., BöhningGaese, K., \& Schleuning, M. (2019). Projecting consequences of global warming for the functional diversity of fleshy-fruited plants and frugivorous birds along a tropical elevational gradient. Diversity and Distributions, 25, 1362-1374.

Schleuning, M., Neuschulz, E. L., Albrecht, J., Bender, I. M., Bowler, D. E., Dehling, D. M., Fritz, S. A., Hof, C., Mueller, T., Nowak, L., Sorensen, M. C., Böhning-Gaese, K., \& Kissling, W. D. (2020). Trait-based assessments of climate-change impacts on interacting Species. Trends in Ecology \& Evolution, 35, 319-328.

Bender, I. M. A., Kissling, W. D., Böhning-Gaese, K., Hensen I., Kühn, I., Nowak, L., Töpfer, T., Wiegand, T., Dehling, D. M., \& Schleuning, M. (2019). Projected impacts of climate change on functional diversity of frugivorous birds along a tropical elevational gradient. Scientific Reports, 9, 17708.

Bender, I. M. A., Kissling, W. D., Blendinger, P. G., Böhning-Gaese, K., Hensen I., Kühn, I., Muñoz, M. C., Neuschulz, E. L. , Nowak, L., Quitián, M., Saavedra, F., Santillán, V., Töpfer, T., Wiegand, T., Dehling, D. M., \& Schleuning, M. (2018). Morphological trait matching shapes plant-frugivore networks across the Andes. Ecography, 41, 1910-1919.

Dugger, P. J., Blendinger, P. G., Böhning-Gaese, K., Chama, L., Correia, M., Dehling, D. M., Emer, C., Farwig, N., Fricke E. C., Galetti, M., García, D., Grass, I., Heleno, R., Jacomassa, F. A. F., Moraes, S., Moran, Catherine, Muñoz, M. C., Neuschulz, E. L., Nowak, L. , Piratelli, A., Pizo, M. A., Quitián, M., Rogers, H. S., Ruggera, R. A., Saavedra, F., Sánchez, M. S., Sánchez, R., Santillán, V., Schabo, D. G., Ribeiro da Silva, F., Timóteo, S., Traveset, A., Vollstädt, M. G. R., \& Schleuning, M. (2018). Seed-dispersal networks are more specialized in the Neotropics than in the Afrotropics. Global Ecology and Biogeography, 28, 248-261.

\section{Submitted manuscripts}

Nowak, L., Schleuning, M., Bender, I. M. A., Böhning-Gaese, K., Dehling, D. M., Fritz, S. A., Kissling, W. D., Müller, T., Neuschulz, E. L., Pigot, A., Sorensen, M. C., Donoso, I. (submitted) Avian seed dispersal may be insufficient for plants to track future temperature change on tropical mountains.

Nowak, L., Schleuning, M., Bender, I. M. A., Kissling, W. D., Fritz, S. A. (submitted) Independent variation of avian sensitivity to climate change and adaptive capacity along a tropical elevational gradient. 


\section{Presentations at conferences and in research institutes}

Presentation at Festival Of Ecology, British Ecological Society Annual Meeting, online

'Avian seed dispersal may be insufficient for plants to track future temperature change on a tropical mountain' - talk

2020 Presentation at University of Amsterdam, Netherlands Biogeography \& Macroecology (BIOMAC) lab of Dr W. Daniel Kissling 'Avian seed dispersal may be insufficient for plants to track future temperature change on a tropical mountain' - talk

2019 Presentation at Universidad Nacional de San Antonio Abad del Cusco, Peru in collaboration with CREES

'Projecting consequences of global warming for the functional diversity of fleshy-fruited plants and frugivorous birds along a tropical elevational gradient' - talk

2019 SIG Biogeography, Frankfurt am Main, Germany 'A multi-trait assessment of species' vulnerability to climate change along a tropical elevational gradient' - poster presentation

$20199^{\text {th }}$ Biennial Conference of the International Biogeography Society, Malaga, Spain

'A multi-trait assessment of species' vulnerability to climate change along a tropical elevational gradient' - poster presentation

2017 Ecology Across Borders, Joint Annual Meeting 2017, Gent, Belgium 'Functional (mis)match of fleshy-fruited plants and frugivorous birds under climate change?' - poster presentation

2017 Presentation at University of Amsterdam, Netherlands Biogeography \& Macroecology (BIOMAC) lab of Dr W. Daniel Kissling 'Functional (mis)match of fleshy-fruited plants and frugivorous birds under climate change?' - talk 